\title{
Momentum broadening in weakly coupled quark-gluon plasma (with a view to finding the quasiparticles within liquid quark-gluon plasma)
}

\author{
Francesco D’Eramo, ${ }^{a, b, c}$ Mindaugas Lekaveckas, ${ }^{a}$ Hong Liu ${ }^{a}$ and Krishna Rajagopal ${ }^{a, d}$ \\ ${ }^{a}$ Center for Theoretical Physics, Massachusetts Institute of Technology, \\ 77 Massachusetts Avenue, Cambridge, MA 02139, U.S.A. \\ ${ }^{b}$ Center for Theoretical Physics, Department of Physics, University of California, \\ 366 Le Conte Hall \#7300, Berkeley, CA 94720, U.S.A. \\ ${ }^{c}$ Theoretical Physics Group, Lawrence Berkeley National Laboratory, \\ 1 Cyclotron Rd. Mail Stop 50A-5104, Berkeley, CA 94720, U.S.A. \\ ${ }^{d}$ Physics Department, Theory Unit, CERN, \\ Case C01600, CH-1211 Genève 23, Switzerland \\ E-mail: fraderamo@berkeley.edu, lekaveck@mit.edu, hong_liu@mit.edu, \\ krishna@mit.edu
}

ABSTRACT: We calculate $P\left(k_{\perp}\right)$, the probability distribution for an energetic parton that propagates for a distance $L$ through a medium without radiating to pick up transverse momentum $k_{\perp}$, for a medium consisting of weakly coupled quark-gluon plasma. We use full or HTL self-energies in appropriate regimes, resumming each in order to find the leading large- $L$ behavior. The jet quenching parameter $\hat{q}$ is the second moment of $P\left(k_{\perp}\right)$, and we compare our results to other determinations of this quantity in the literature, although we emphasize the importance of looking at $P\left(k_{\perp}\right)$ in its entirety. We compare our results for $P\left(k_{\perp}\right)$ in weakly coupled quark-gluon plasma to expectations from holographic calculations that assume a plasma that is strongly coupled at all length scales. We find that the shape of $P\left(k_{\perp}\right)$ at modest $k_{\perp}$ may not be very different in weakly coupled and strongly coupled plasmas, but we find that $P\left(k_{\perp}\right)$ must be parametrically larger in a weakly coupled plasma than in a strongly coupled plasma - at large enough $k_{\perp}$. This means that by looking for rare (but not exponentially rare) large-angle deflections of the jet resulting from a parton produced initially back-to-back with a hard photon, experimentalists can find the weakly coupled short-distance quark and gluon quasiparticles within the strongly coupled liquid quark-gluon plasma produced in heavy ion collisions, much as Rutherford found nuclei within atoms or Friedman, Kendall and Taylor found quarks within nucleons.

KeYwords: Heavy Ion Phenomenology, Jets

ARXIV EPRINT: 1211.1922 


\section{Contents}

1 Introduction 1

$\begin{array}{lll}2 & \text { Setting up the formalism } & 6\end{array}$

3 Retarded gluon propagator 12

4 Breakdown of perturbation theory and self-energy matching 18

5 Results and discussion $\quad 21$

5.1 Results for a thin medium, and comparison to previous results 22

5.2 Complete results for the probability distribution $P\left(k_{\perp}\right)$

5.3 Jet quenching parameter 30

5.4 From weak to strong coupling 35

6 Outlook 40

A Schwinger-Keldysh formalism 43

B A thin medium $\quad 45$

$\begin{array}{ll}\text { C Exponentiation of } \mathcal{W}_{\mathcal{R}}^{(2)} & 47\end{array}$

$\begin{array}{ll}\text { D Boltzmann equation approach } & 48\end{array}$

$\begin{array}{ll}\text { E A tensor basis for the self-energy } & 50\end{array}$

$\begin{array}{lll}\text { F } & \text { Resumming AGZ } & \mathbf{5 0}\end{array}$

G Simple formula to compute $\hat{q} \quad 54$

\section{Introduction}

The droplets of quark-gluon plasma (QGP) produced in heavy-ion collisions can be studied via analyzing the "shape" (in various senses including almost literally) of the explosion of hadrons it decays into and via analyzing their effects on various internally generated "probes". High energy partons, produced in hard parton-parton scattering events occurring at the earliest moments within a heavy ion collision, are particularly useful probes. After their production they will propagate through as much as 5-10 fm of the hot and dense medium produced in the collision. In proton-proton collisions, the production rates and decay products (jets) of such high energy partons are well measured and well understood, 
meaning that in heavy ion collisions they constitute well calibrated probes of the plasma. The suite of observables that indicate the ways in which high energy partons interact with the plasma, for example losing energy to it, are collectively known as "jet quenching". The suppression of the production rates for high- $p_{T}$ hadrons in heavy ion collisions at RHIC with respect to expectations based on scaling with the number of binary nucleon-nucleon collisions was the earliest manifestation of jet quenching to be discovered [1-4]. Jet quenching manifests itself in many observables, which contain clues about how the fragmentation of a high energy parton is affected by the presence of the plasma and how the medium responds to the energy and momentum that a fragmenting parton transfers to it. Jet quenching has by now been seen in many ways, both at RHIC and at the LHC. At the LHC, jet energies are high enough that the jets can be detected calorimetrically event-by-event, and the phenomenon of jet quenching is manifest in single events with, say, a jet with an energy greater than $200 \mathrm{GeV}$ back-to-back with a jet with an energy less than $100 \mathrm{GeV}$ [5-12]. (It is improbable that a pair of jets will be produced such that each travels the same distance through the plasma and loses the same amount of energy, so back-to-back pairs of jets with unbalanced energies are the norm.) The first measurements of heavy ion collisions at the LHC in which a hard parton (manifest in the final state as a jet) was produced back-to-back with a single hard photon have recently been reported [10, 13-18], and early results on energetic hadrons back-to-back with a single hard photon in heavy ion collisions at RHIC have also been reported $[19,20]$. Since the photon tells us the initial transverse momentum and direction of the parton that produced the jet, analyzing sufficiently large data sets of such events can tell us how the plasma produced in heavy ion collisions affects the energy, fragmentation, and direction of hard partons plowing through it.

Theoretical analyses of how the energy and momenta of hard partons are modified by passage through weakly coupled quark-gluon plasma have been developed by many authors [21-36] and are reviewed in refs. [37-44]. In the limit of high parton energy, parton energy loss occurs dominantly via the radiation of nearly collinear gluons, an effect that is distinct from the changes in the direction of the momentum of the hard parton via (repeated, soft) elastic collisions. The latter effect is often called "transverse momentum broadening", where the word "transverse" here and throughout the remainder of this paper means perpendicular to the original direction of the energetic parton, not perpendicular to the beam direction. Transverse momentum broadening describes the accumulation of changes to the direction of the momentum of the hard parton as it propagates through a medium. Transverse momentum broadening plays a central role in all the calculations of radiative energy loss, since the incoming and outgoing partons and the radiated gluons are all continually being jostled by the medium in which they find themselves.

Analysis of an energetic parton propagating through a medium immediately involves (at least) two well-separated energy scales, $Q \gg T$, where $Q$ is the energy of the hard parton and $T$ refers to any of the soft scales that characterize the medium itself. In the case of weakly coupled quark-gluon plasma, " $T$ " could refer to the temperature $T$ itself or to even softer scales like $g T$ or $g^{2} T$, with $g$ the QCD coupling. This separation of scales suggests the use of Effective Field Theory (EFT), which takes advantage of a separation of energy scales to simplify calculations and enhance predictive power by identifying relevant degrees of free- 
dom, simplifications, or new symmetries that appear in the limit of large scale separation. Our long-term goal is to use EFT techniques to develop controlled theoretical calculations of the propagation of energetic particles through hot and dense media. The hierarchy $Q \gg T$ makes Soft Collinear Effective Theory (SCET) [45-48], the natural EFT with which to describe hard partons in a medium. (SCET was introduced in other contexts in which both energetic (collinear) partons and soft degrees of freedom are relevant.) The work of ref. [49], which builds upon the earlier analysis of transverse momentum broadening in ref. [36], was the first use of SCET to analyze partons in medium. These authors looked at the transverse momentum broadening of partons produced in deep inelastic scattering on large nuclei. In particular, they discovered that the transverse momentum broadening of an energetic parton is induced by its interactions with the gluons from the medium in a particular kinematic regime, known as "Glauber gluons", that were not present in the original formulation of SCET. Upon choosing coordinate axes such that the three-momentum of the energetic parton is initially along the negative $z$-axis, meaning that its initial four-momentum is

$$
q_{0} \equiv\left(q_{0}^{+}, q_{0}^{-}, q_{0 \perp}\right)=(0, Q, 0) .
$$

(in light-cone coordinates defined by $q^{ \pm}=\frac{1}{\sqrt{2}}\left(q^{0} \pm q^{3}\right)$ ) Glauber gluons are gluons whose momenta are parametrically of order [50]

$$
\left(\frac{T^{2}}{Q}, \frac{T^{2}}{Q}, T\right)
$$

After absorbing or emitting any number of Glauber gluons, the momentum of the energetic parton is parametrically of order $\left(T^{2} / Q, Q, T\right)$, meaning that it is off-shell only by of order $T^{2}$ and so does not radiate either collinear or soft gluons [50]. And yet, repeated absorption and emission of Glauber gluons continually kicks the hard parton and can result in significant transverse momentum broadening. In ref. [50], three of us developed a SCET formulation for the calculation of transverse momentum broadening in any medium, accounting for arbitrarily many interactions between the energetic parton and the Glauber modes from the medium. SCET has also been used to study momentum broadening and collinear gluon radiation to first order in opacity in ref. [51, 52]. In this paper, we evaluate the general result of [50] for the specific case of a weakly coupled quark-gluon plasma, in QCD, in thermal equilibrium.

Transverse momentum broadening is described by $P\left(k_{\perp}\right)$, the probability density that after propagating through the medium for a distance $L$ without radiating, the energetic parton has acquired momentum transverse to its initial direction given by $k_{\perp}$. The probability distribution is normalized as

$$
\int \frac{d^{2} k_{\perp}}{(2 \pi)^{2}} P\left(k_{\perp}\right)=1
$$

$P\left(k_{\perp}\right)$ depends on the medium length $L$, but we will keep this dependence implicit in our notation. $P\left(k_{\perp}\right)$ is obtained by summing over infinitely many Feynman diagrams, accounting for the interactions between the energetic parton with any number of Glauber gluons from the medium. In ref. [50] this calculation was performed by treating the Glauber 
gluons as background fields, analyzing the propagation of the energetic parton in the presence of any background field configuration, and then averaging the result over all possible background field configurations.

The final result for the differential probability distribution reads [50]

$$
P\left(k_{\perp}\right)=\int d^{2} x_{\perp} e^{-i k_{\perp} \cdot x_{\perp}} \mathcal{W}_{\mathcal{R}}\left(x_{\perp}\right),
$$

where $\mathcal{R}$ is the $\mathrm{SU}\left(N_{c}\right)$ representation to which the energetic particle belongs and $\mathcal{W}_{\mathcal{R}}\left(x_{\perp}\right)$ is the expectation value of two light-like Wilson lines with spatial extent $L$ (and therefore length $L^{-} \equiv \sqrt{2} L$ along the light cone) in representation $\mathcal{R}$ separated from each other in the transverse plane by the vector $x_{\perp} .{ }^{1}$ The explicit definition of $\mathcal{W}_{\mathcal{R}}\left(x_{\perp}\right)$ is given at the beginning of section 2. The expression (1.4) was obtained previously using different methods in refs. [41, 53]. The nature of the medium - for example whether it is weakly coupled or strongly coupled - does not enter in the analysis of the propagation in any one background field configuration, and therefore does not affect the expression (1.4) [50]. This distinction, or indeed any property of the medium, only becomes relevant when one averages over all possible background field configurations, which is to say when one evaluates the expectation value $\mathcal{W}_{\mathcal{R}}\left(x_{\perp}\right)$. If the medium of interest is in thermal equilibrium, the expectation value $\mathcal{W}_{\mathcal{R}}\left(x_{\perp}\right)$ is a thermal average that can be evaluated in equilibrium thermal field theory. If the medium of interest is not in equilibrium, the expectation value $\mathcal{W}_{\mathcal{R}}\left(x_{\perp}\right)$ is much harder to evaluate but the expression (1.4) remains correct. If the medium of interest is cold nuclear matter, for example in approaches to understanding the Cronin effect in proton-nucleus collisions via transverse momentum broadening of the incident parton from the proton before its hard scattering [54-56], then the relevant $P\left(k_{\perp}\right)$ is also given by (1.4). In all these contexts, the result (1.4) is valid only in the high energy limit for the propagating parton. Specifically, it requires that $Q \gg k_{\perp}^{2} L$, as this is the criterion that ensures that the trajectory of the hard parton in position space remains well-approximated as a straight line, even as the parton picks up transverse momentum $k_{\perp}$. In this limit, $P\left(k_{\perp}\right)$ is given by (1.4) and is therefore independent of the energy of the hard probe, depending only on the properties of the medium through the thermal expectation value $\mathcal{W}_{\mathcal{R}}\left(x_{\perp}\right)$. Transverse momentum broadening without radiation thus "measures" a field-theoretically well-defined property of the medium.

In ref. [50], an explicit evaluation of the thermal average $\mathcal{W}_{\mathcal{R}}\left(x_{\perp}\right)$ and hence $P\left(k_{\perp}\right)$ was provided only for the plasma of $\mathcal{N}=4$ supersymmetric Yang-Mills (SYM) theory in the large number of colors $\left(N_{c}\right)$ and strong coupling limit, in which this strongly coupled plasma has a holographic description, allowing the calculation to be done via gauge/gravity

\footnotetext{
${ }^{1}$ The expression (1.4) is gauge invariant only after the ends of the lightlike Wilson lines are closed with transverse segments, completing a Wilson loop. However, in the limit $L \gg 1 / T$ the contribution of these transverse segments is subleading in any covariant gauge, meaning that the gauge invariant result can be obtained by evaluating (1.4), which includes only the two long lightlike Wilson lines, in any covariant gauge [50]. In lightcone gauge, the expectation value of the lightlike lines vanishes and the same result is obtained entirely from the transverse segments $[50,57]$. In the present paper, we shall work in a covariant gauge, obtaining the gauge invariant result directly from (1.4).
} 
duality. ${ }^{2}$ In this plasma (and, quite likely in any plasma in the strong coupling limit) $P\left(k_{\perp}\right)$ is Gaussian in $k_{\perp}$ and transverse momentum broadening can be understood as diffusion in $k_{\perp}$-space due to repeated, even continuous, interactions between the hard parton and the strongly coupled medium.

In this work, we shall consider a QCD plasma in equilibrium at high enough temperatures that physics at scales $\sim T$ is weakly coupled. We shall evaluate the thermal average $\mathcal{W}_{\mathcal{R}}\left(x_{\perp}\right)$ perturbatively, using standard methods from thermal field theory. From this we then obtain $P\left(k_{\perp}\right)$ and hence momentum broadening in a weakly coupled quark-gluon plasma by applying (1.4). We shall present this calculation over the course of sections 2,3 and 4 . We set up the general formalism and identify the leading order contribution in the gauge coupling $g$ (assumed $\ll 1$ ) to the expression in $(1.4)$. In doing so, we resum an infinite class of diagrams which are enhanced by the medium length $L$. For a thick medium, the resummation alters $P\left(k_{\perp}\right)$ at small $k_{\perp}$, as we will show explicitly. Once we have set up the formalism and identified the expression that we need to evaluate, we find that $P\left(k_{\perp}\right)$ depends on the retarded gluon propagator. In section 3 , we show how this propagator can be expressed in terms of self-energies, which we compute. We compute the self-energies first using ordinary perturbation theory, for any value of the external momentum. Since our goal is to compute the probability distribution in (1.4), which manifestly depends on a gluon correlator in coordinate space, we need the gluon propagator for any value of the external momentum. Famously, perturbation theory for non-Abelian gauge theories at finite temperature breaks down in the infrared [59,60]. In the case of the gluon propagator this happens when the external momentum is of order $g^{2} T$, and we recover this pathology from our expression. We take care of the infrared problem by using the hard thermal loop (HTL) effective theory [61-65], which is valid for momenta of order $g T$ and below and which restores a consistent perturbative expansion. We work in the weak-coupling regime, where the hierarchy $g^{2} T \ll g T$ guarantees that the infrared problem does not show up in our calculation. In section 4 we discuss this in detail, and we explain how we use full or HTL retarded self-energies in the appropriate regimes, as well as how we match them at an intermediate scale. The reader only interested in our results, not in their derivation, can jump directly to section 5 . There we present our results, compare them to results at strong coupling [50, 58], and compare them to other weak-coupling results in the literature for $P\left(k_{\perp}\right)$ and its second moment, which is called the jet quenching parameter $\hat{q}[36,66-69]$.

The most important qualitative feature of our result is that $P\left(k_{\perp}\right) \propto 1 / k_{\perp}^{4}$ in the large $k_{\perp}$ regime. Since this is parametrically larger than a Gaussian at large $k_{\perp}$ this means that at sufficiently large $k_{\perp} P\left(k_{\perp}\right)$ is greater in a weakly coupled plasma than in a strongly coupled one. We close section 5 by exploring this comparison, semi-quantitatively. In section 6 we conclude and look ahead. We do not expect that our result for weakly coupled quark-gluon plasma describes $P\left(k_{\perp}\right)$ for quark-gluon plasma produced in heavy ion collisions correctly at all $k_{\perp}$. Heavy ion collisions do not reach the asymptotically high temperatures at which $g \ll$ 1 at scales of order the temperature. Instead, there are many indications that the plasma

\footnotetext{
${ }^{2}$ The result so obtained [50] confirms one obtained first in ref. [58], and eliminates certain subtleties in the earlier derivation.
} 


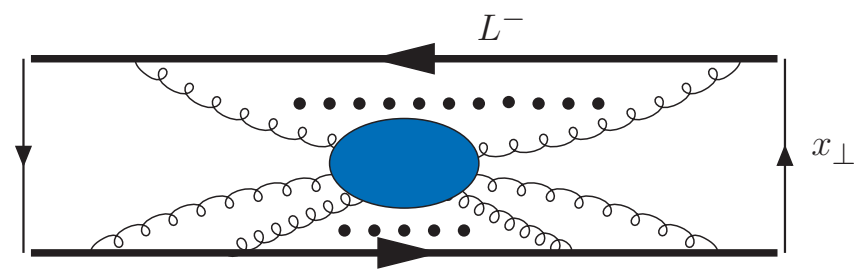

Figure 1. Diagrams contributing to the expectation value of the Wilson loop, $\mathcal{W}_{\mathcal{R}}\left(x_{\perp}\right)$. The length of each of the long light-like sides of the loop is $L^{-}=\sqrt{2} L$. The light-like Wilson lines are separated in the transverse direction by a distance $x_{\perp}$.

produced in heavy ion collisions at both RHIC and LHC is a strongly coupled liquid, with no well-defined quasiparticles (i.e. no quasiparticles with mean free paths long compared to $1 / T)$. At small $k_{\perp}$, we therefore expect that its $P\left(k_{\perp}\right)$ is more similar to that in the strongly coupled $\mathcal{N}=4 \mathrm{SYM}$ plasma $[50,58]$ than to that we calculate in this paper. However, QCD is asymptotically free meaning that at short enough distance scales the strongly coupled liquid must be described by weakly interacting quark and gluon quasiparticles. We do not incorporate the running of $g$ with $k_{\perp}$ in our calculation. Nevertheless we expect that, because $g$ does run, $P\left(k_{\perp}\right)$ for the strongly coupled liquid produced in heavy ion collisions is well described at large enough $k_{\perp}$ by the result of our weakly coupled calculation of $P\left(k_{\perp}\right)$. This means that if $P\left(k_{\perp}\right)$ can be measured over a sufficiently wide range of $k_{\perp}$ it could yield insights into how quark-gluon plasma with liquid-like properties at length scales of order $1 / T$ emerges from a weakly coupled gauge theory at short distances.

\section{Setting up the formalism}

In this section, we derive an expression valid to leading order in the QCD coupling constant, and hence in a weakly coupled QCD plasma, relating the general result for $P\left(k_{\perp}\right)$, derived in ref. [50] and given in (1.4), to the retarded gluon propagator. We see in (1.4) that the probability distribution $P\left(k_{\perp}\right)$ that describes momentum broadening is the Fourier transform of $\mathcal{W}_{\mathcal{R}}\left(x_{\perp}\right)$, the expectation value of two light-like Wilson lines in representation $\mathcal{R}$ separated from each other by a distance $x_{\perp}$ in the transverse plane. We begin by defining $\mathcal{W}_{\mathcal{R}}\left(x_{\perp}\right)$ explicitly:

$$
\mathcal{W}_{\mathcal{R}}\left(x_{\perp}\right) \equiv \frac{1}{d(\mathcal{R})}\left\langle\operatorname{Tr}\left[W_{\mathcal{R}}^{\dagger}\left[0, x_{\perp}\right] W_{\mathcal{R}}[0,0]\right]\right\rangle
$$

where each Wilson line along the light-cone is in turn defined by

$$
W_{\mathcal{R}}\left[y^{+}, y_{\perp}\right] \equiv P\left\{\exp \left[i g \int_{0}^{L^{-}} d y^{-} A_{\mathcal{R}}^{+}\left(y^{+}, y^{-}, y_{\perp}\right)\right]\right\} \text {. }
$$

Next, we Taylor expand (2.1), and in doing so we define

$$
\mathcal{W}_{\mathcal{R}}\left(x_{\perp}\right) \equiv 1+\sum_{j=2}^{\infty} \mathcal{W}_{\mathcal{R}}^{(j)}\left(x_{\perp}\right)=1+\mathcal{W}_{\mathcal{R}}^{(2)}+\mathcal{W}_{\mathcal{R}}^{(3)}+\ldots
$$


where $\mathcal{W}_{\mathcal{R}}^{(j)}$ denotes the contribution in which $j$ gluon fields are evaluated at points on the light-like Wilson lines or, equivalently, the contribution from those diagrams (see figure 1) in which gluon propagators end at $j$ vertices on the light-like Wilson lines. We have dropped the explicit denotation of the $x_{\perp}$-dependence on the right-hand side of (2.3), and will do so at many points below. Each term on the right-hand side of (2.3) is itself a series,

$$
\mathcal{W}_{\mathcal{R}}^{(j)}=\sum_{k=0}^{j} W_{\mathcal{R}}^{(k, j-k)}
$$

where the contribution from a diagram with $k$ gluon vertices on the light-like line at the perpendicular position $x_{\perp}$ and $j-k$ gluon vertices on the light-like line at the origin of the perpendicular plane is given by

$$
\begin{aligned}
W_{\mathcal{R}}^{(k, j-k)}= & \frac{(-i)^{k} i^{j-k} g^{j}}{d(\mathcal{R}) k !(j-k) !} \int_{0}^{L^{-}} d y_{1}^{-} \ldots d y_{j}^{-} \\
& \times\left\langle\operatorname{Tr}\left[\mathcal{P}\left\{A_{\mathcal{R}}^{+}\left(y_{1}^{-}, x_{\perp}\right) \ldots A_{\mathcal{R}}^{+}\left(y_{k}^{-}, x_{\perp}\right) A_{\mathcal{R}}^{+}\left(y_{k+1}^{-}, 0_{\perp}\right) \ldots A_{\mathcal{R}}^{+}\left(y_{j}^{-}, 0_{\perp}\right)\right\}\right]\right\rangle .
\end{aligned}
$$

Here, $g$ is the $\mathrm{SU}\left(N_{c}\right)$ gauge coupling constant, $\mathcal{P}$ stands for path ordering of the gluon fields and the trace is taken over $\mathrm{SU}\left(N_{c}\right)$ color indices. We need not specify the $y^{+}$coordinates at which the gluon fields in (2.5) are evaluated because they are evaluated on the light-cone described by varying $y^{-}$at fixed $y^{+}$, and we can use the translational invariance of the medium to set all of the $y^{+}$coordinates to 0 . The gluon fields in (2.5) can each be written as the product of an operator and a group matrix: $A_{\mathcal{R}}^{+}=A^{a+} t_{\mathcal{R}}^{a}$. Note, finally, that since the expectation value of a single gluon field vanishes, there is no $j=1$ contribution in the expansion (2.3). We have now specified the $\mathcal{W}_{\mathcal{R}}$ of $(2.1)$ fully explicitly. The probability distribution $P\left(k_{\perp}\right)$ that describes momentum broadening is then given by the Fourier transform of $\mathcal{W}_{\mathcal{R}}$, as in (1.4).

Both the gluon operators $A^{a+}$ and the group matrices $t_{\mathcal{R}}^{a}$ within $\mathcal{W}_{\mathcal{R}}$ are ordered along the path as indicated by arrows in figure 1, in contrast with the time ordered operators in a conventional Wilson loop [50]. Hence, the expectation value $\mathcal{W}_{\mathcal{R}}$ should be described by a Schwinger-Keldysh contour with one light-like Wilson line on the $\operatorname{Im} t=0$ segment of the contour and the other one on the $\operatorname{Im} t=-i \epsilon$ segment. The infinitesimal displacement in imaginary time ensures that the operators from the two lines are ordered such that all operators from one line come before any operators from the other. The SchwingerKeldysh formalism relevant to our calculation is reviewed in appendix A. A typical diagram contributing to $\mathcal{W}_{\mathcal{R}}\left(x_{\perp}\right)$ is shown in figure 1 .

We are looking for the leading order contribution to $\mathcal{W}_{\mathcal{R}}$, leading in the $g \ll 1$ limit. The first non-trivial and non-vanishing contribution appears for $j=2$, and is given by the diagrams in figure 2. Upon writing each gluon field as $A_{\mathcal{R}}^{+}=A^{a+} t_{\mathcal{R}}^{a}$, the propagator that arises in $\mathcal{W}_{\mathcal{R}}^{(2)}$ reads

$$
\left\langle\operatorname{Tr}\left[A_{\mathcal{R}}^{+}\left(y_{1}^{-}, y_{1 \perp}\right) A_{\mathcal{R}}^{+}\left(y_{2}^{-}, y_{2 \perp}\right)\right]\right\rangle=\operatorname{Tr}\left[t_{\mathcal{R}}^{a} t_{\mathcal{R}}^{b}\right]\left\langle A^{a+}\left(y_{1}^{-}, y_{1 \perp}\right) A^{b+}\left(y_{2}^{-}, y_{2 \perp}\right)\right\rangle
$$



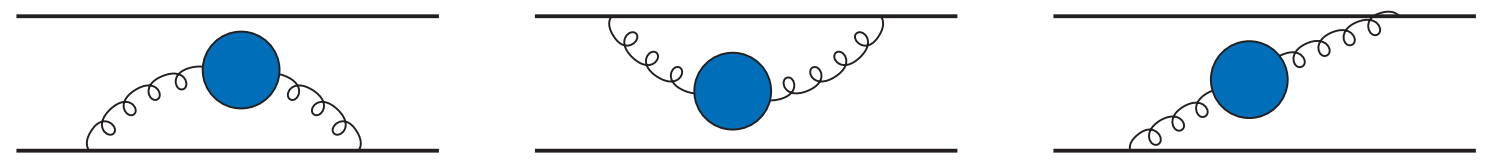

Figure 2. Diagrams contributing to $\mathcal{W}_{\mathcal{R}}^{(2)}$. Here, the blue blobs stand for full interacting gluon two-point Green functions.

where $y_{1 \perp}$ and $y_{2} \perp$ can each be either $0_{\perp}$ or $x_{\perp}$. The expectation value on the right-hand side of (2.6) is diagonal in the color indices, making it convenient to define

$$
\left\langle A^{a+}\left(y_{1}^{-}, y_{1 \perp}\right) A^{b+}\left(y_{2}^{-}, y_{2 \perp}\right)\right\rangle \equiv \delta^{a b} D^{>}\left(y_{1}^{-}-y_{2}^{-}, y_{1 \perp}-y_{2 \perp}\right),
$$

where the Wightman propagator $D^{>}$has no color indices. ${ }^{3}$ We can now write

$$
\left\langle\operatorname{Tr}\left[A_{\mathcal{R}}^{+}\left(y_{1}^{-}, y_{1 \perp}\right) A_{\mathcal{R}}^{+}\left(y_{2}^{-}, y_{2 \perp}\right)\right]\right\rangle=d(\mathcal{R}) C_{\mathcal{R}} D^{>}\left(y_{1}^{-}-y_{2}^{-}, y_{1 \perp}-y_{2 \perp}\right)
$$

where we have identified the quadratic Casimir factor $C_{\mathcal{R}}$ for the $\mathrm{SU}\left(N_{c}\right)$ representation $\mathcal{R}$ via the relation

$$
\delta^{a b} t_{\mathcal{R}}^{a} t_{\mathcal{R}}^{b}=C_{\mathcal{R}} \mathbf{I}_{\mathcal{R}}
$$

where $\mathbf{I}_{\mathcal{R}}$ is the identity matrix for the representation $\mathcal{R}$. With all these definitions in place, $\mathcal{W}_{\mathcal{R}}^{(2)}$ now reads

$$
\mathcal{W}_{\mathcal{R}}^{(2)}=-g^{2} C_{\mathcal{R}} \int_{0}^{L^{-}} d y_{1}^{-} \int_{0}^{L^{-}} d y_{2}^{-} \times\left[D^{>}\left(y_{1}^{-}-y_{2}^{-}, 0_{\perp}\right)-D^{>}\left(y_{1}^{-}-y_{2}^{-}, x_{\perp}\right)\right]
$$

where $L^{-}=\sqrt{2} L$. Finally, we perform a change of variables for the integrations over the $y^{-}$coordinates, defining

$$
Y^{-} \equiv \frac{y_{1}^{-}+y_{2}^{-}}{2}, \quad y^{-} \equiv y_{1}^{-}-y_{2}^{-} .
$$

The integration over the "center of mass" coordinate $Y^{-}$is straightforward, and we find

$$
\mathcal{W}_{\mathcal{R}}^{(2)}=-g^{2} C_{\mathcal{R}} L^{-} \int d y^{-}\left[D^{>}\left(y^{-}, 0_{\perp}\right)-D^{>}\left(y^{-}, x_{\perp}\right)\right]
$$

In order to determine the leading gauge coupling dependence of $\mathcal{W}_{\mathcal{R}}^{(2)}$, we need to determine how the gluon propagator depends on $g$. The tree level term in the gluon propagator is proportional to the metric tensor $g^{\mu \nu}$, and since $g^{++}=0$ this vanishes. The first non-vanishing contribution is found at next order in perturbation theory, when we evaluate the one-loop propagator - i.e. replacing the blue blobs in figure 2 by single loops. It is straightforward to count the powers of $g$ when the external momentum in the

\footnotetext{
${ }^{3}$ It would be more precise to call the propagator $D^{>++}$, since we only have the + component of the gluon fields. We omit the ++ for notational simplicity. However, we keep the symbol $>$ to remind ourselves that this is the Wightman propagator. We shall later relate $D^{>}$to the retarded propagator.
} 
propagator (i.e. momentum in the gluon lines shown explicitly in figure 2) are greater than the scale $g T$. In this case, conventional perturbation theory is under control, the one-loop propagator is $\mathcal{O}\left(g^{2}\right)$ and the full expression in (2.12) is $\mathcal{O}\left(g^{4}\right)$. For external momentum in the propagator of the order $g T$ and lower, we shall see below that HTL resummation is necessary. We shall complete the discussion of the $g$-dependence of our results in section 5 .

Before we move on, we need to check that the $\mathcal{W}_{\mathcal{R}}^{(j)}$ for $j>2$ are suppressed relative to $\mathcal{W}_{\mathcal{R}}^{(2)}$. Each gluon field from the Wilson lines brings a factor of $g$ with it, meaning that $\mathcal{W}_{\mathcal{R}}^{(j)}$ comes with a factor of $g^{j}$ from attaching $j$ gluons to the Wilson lines. This, together with the fact that $\mathcal{W}_{\mathcal{R}}^{(3)}$ must include a three-gluon vertex that comes with another $g$, suggests that all the $\mathcal{W}_{\mathcal{R}}^{(j)}$ for $j>2$ are suppressed relative to $\mathcal{W}_{\mathcal{R}}^{(2)}$ by a factor of at least $g^{2}$. The only way to avoid this conclusion would be if the tree-level contribution to $\mathcal{W}_{\mathcal{R}}^{(3)}$ or $\mathcal{W}_{\mathcal{R}}^{(4)}$ were nonzero, since we saw that $\mathcal{W}_{\mathcal{R}}^{(2)}$ vanishes at tree-level. However, because the three-point gluon vertex has the form $g^{\mu \nu} p^{\rho}$, where $p^{\rho}$ is one of the incoming gluon momenta, and because $g^{++}=0$, the tree-level contribution to $\mathcal{W}_{\mathcal{R}}^{(3)}$ vanishes. It is also straightforward to check that the tree-level contribution to $\mathcal{W}_{\mathcal{R}}^{(4)}$ vanishes.

We conclude that the contribution to the series in (2.3) that is leading order in powers of $g$ is given by $\mathcal{W}_{\mathcal{R}}^{(2)}$, which is related to the gluon propagator $D^{>}$according to (2.12).

The formalism from ref. [50] within which (1.4) was derived requires $L T \gg 1$. If we were to require in addition that $g^{2} C_{\mathcal{R}} L T \ll 1$, which is satisfied at weak enough coupling for any given $L$, we would have achieved our goal for this section having derived the relationship (2.12). However, we would prefer to have a result that is valid at large $L$ for any given weak value of the coupling $g$. For this purpose the leading order contribution to $\mathcal{W}_{\mathcal{R}}$ given by (2.12) does not suffice because it is proportional to the length of the medium $L$, meaning that if we evaluate $P\left(k_{\perp}\right)$ by taking the Fourier transform of (2.12) as prescribed in (1.4), we obtain a probability distribution that is proportional to $L$. This cannot be the correct result at large enough $L$ for any fixed $g$. In particular, we find that when $g^{2} C_{\mathcal{R}} L T \sim 1$ or greater, the perturbative expansion is not under control. In appendix B we complete the discussion of a "thin medium", in which $g^{2} C_{\mathcal{R}} L T \ll 1$, showing how in this circumstance (2.12) yields a correctly normalized probability distribution $P\left(k_{\perp}\right)$. Here, we shall do better.

In order to find a perturbative expansion that is valid for any value of $L$ (and which of course reduces to what we have already derived if $g^{2} C_{\mathcal{R}} L T$ is $\ll 1$ ) we need to consider the $L$-dependence of each diagram contributing in (2.3). In a translation-invariant medium, each contribution to the series (2.3) is, at minimum, proportional to $L$. This can easily be seen by starting from the definition in (2.3) and changing the $y^{-}$coordinates to a new set including the center-of-mass coordinate $Y^{-}$, in analogy to what we have done for $\mathcal{W}_{\mathcal{R}}^{(2)}$ in (2.11). The gluon correlator cannot depend on the center of mass position, and therefore the integration along $Y^{-}$is straightforward, giving just a factor of $L$. If this were the complete story, we would (incorrectly) conclude that power counting in $g$ and $L$ results in

$$
\mathcal{W}_{\mathcal{R}}^{(j)} \propto g^{r_{j}} L, \quad r_{j} \geq j,
$$

and would further conclude that the previous perturbative expansion needs no modification. In fact, the $L$ power counting in (2.13) is incorrect because its "derivation" assumed 


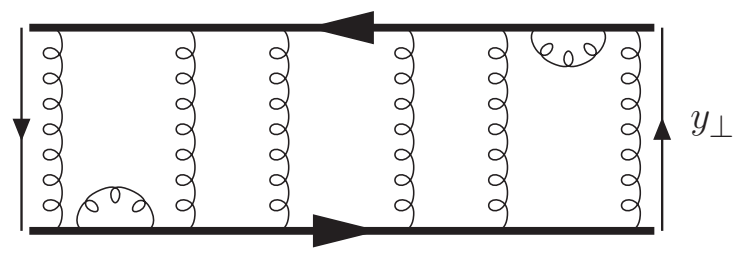

Figure 3. A contraction of $n=j / 2$ gluons, here with $n=8$, giving a contribution proportional to $L^{n}$. This is an example of a diagram whose contribution is length-enhanced.

that the diagram in figure 1 is connected. If this were the case, there would be only one global translational invariance, and therefore one single center of mass integration. Disconnected diagrams, however, always have additional translational invariances (corresponding to the freedom to translate disconnected pieces of the diagram independently) that yield additional integrations over center-of-mass coordinates that in turn result in the contribution of the diagram being enhanced by additional powers of $L$. Thus when $g^{2} C_{\mathcal{R}} L T \sim 1$ or greater we will need to resum a suitable set of disconnected diagrams, namely those whose contributions are the most "length-enhanced". Disconnected diagrams can always be drawn for $j \geq 4$, and the greatest number of translational invariances is reached in diagrams with the greatest number of disconnected pieces.

From the cluster decomposition principle, we expect that when we include all disconnected diagrams, $\mathcal{W}_{\mathcal{R}}\left(x_{\perp}\right)$ can be written in the exponentiated form

$$
\mathcal{W}_{\mathcal{R}}=\exp \left(\sum \text { connected diagrams }\right) .
$$

We emphasize that here connected diagrams include diagrams which could be disconnected were the gauge group Abelian. For example, consider the diagrams in figures 3 and 4 . The first is clearly disconnected. Naively, the cross diagram within figure 4 also appears disconnected in coordinate space. However, the contractions in color space restrict the coordinate space integrations, and the cross diagram should in fact be considered connected. Since all connected diagrams come with precisely one factor of $L$, our earlier power counting of $g$ goes through, but now when applied to the exponent in (2.14). So, to leading order in weak coupling we now have

$$
P\left(k_{\perp}\right)=\int d^{2} x_{\perp} e^{-i k_{\perp} \cdot x_{\perp}} \exp \left[\mathcal{W}_{\mathcal{R}}^{(2)}\right] .
$$

The proof of (2.14) is almost analogous to the textbook proof of the relation between the connected and disconnected diagrams in, say, $\lambda \phi^{4}$ theory. There are some additional complications involving path ordering and group contractions. We illustrate how these are resolved in appendix $\mathrm{C}$, by giving as an example a proof of the exponentiation of $\mathcal{W}_{\mathcal{R}}^{(2)}$, namely (2.15).

We conclude that whereas for a thin medium in which $g^{2} C_{\mathcal{R}} L T \ll 1$ the leading contribution to $P\left(k_{\perp}\right)$ at weak coupling is given by (1.4) with $W_{\mathcal{R}}$ replaced by just $\mathcal{W}_{\mathcal{R}}^{(2)}$, if $g^{2} C_{\mathcal{R}} L T$ is not small we must resum all disconnected diagrams involving only $\mathcal{W}_{\mathcal{R}}^{(2)}$ as in 


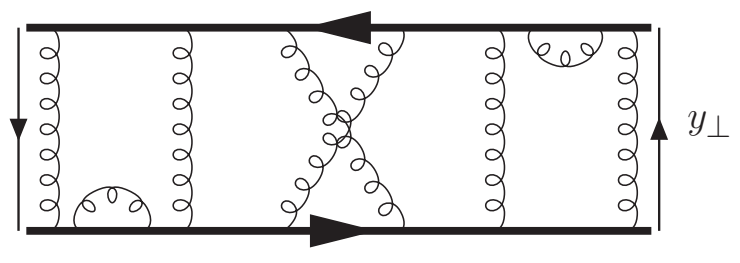

Figure 4. A different contraction of $k=j / 2=8$ gluons giving a contribution which is proportional only to $L^{7}$, making it less "length-enhanced" than that of figure 3 . If we neglect powers of $g$ coming from within the propagators, the cross diagram in this figure gives a factor of $g^{4} L$ which should be compared with the $g^{4} L^{2}$ factor coming from two disconnected lines. This diagram and that of figure 3 give contributions proportional to the same power of $g$ but, among all such contributions, that from figure 3 is one of those that comes with the highest possible power of $L$ while that from this diagram is not. So, the diagram in figure 3 is one of the length-enhanced diagrams that we resum while this diagram is not.

figure 3 , obtaining

$$
\exp \left[\mathcal{W}_{\mathcal{R}}^{(2)}\right]=1+\sum_{n=1}^{\infty} \frac{1}{n !}\left(\mathcal{W}_{\mathcal{R}}^{(2)}\right)^{n}
$$

where $\left(\mathcal{W}_{\mathcal{R}}^{(2)}\right)^{n}$ contains a "length enhancement" factor $L^{n}$. By keeping only the leading order term in the exponent in (2.14) we are resumming those diagrams that have the highest possible power of $L$ for a given power of $g$. This is illustrated in figures 3 and 4 . The diagram in figure 3 is included in our resummation (2.15); it contributes to the $n=8$ term in the expansion (2.16). The diagram in figure 4 arises in (2.14) from a cross-term involving 6 powers of $\mathcal{W}_{\mathcal{R}}^{(2)}$ and one power of $\mathcal{W}_{\mathcal{R}}^{(4)}$. It therefore does not arise in (2.16) or (2.15). It is not included in our resummation because, for its power of $g$, it is less length-enhanced than the diagram in figure 3.

The physical interpretation of resumming length enhanced diagrams is that by doing so we are taking into account the possibility that the energetic parton scatters many times over the course of propagating for a distance $L$ through the medium. In appendix D we show explicitly that the resummation we have performed is equivalent to considering multiple scattering by deriving and solving a Boltzmann equation for momentum broadening. The rederivation of our results in appendix D is also helpful in making contact between our results and those in previous literature. To that end, in appendix D we analyze the Boltzmann equation with a collision kernel that includes only one gluon exchange. The solution to this Boltzmann equation is identical to eq. (2.15), which we obtained by describing processes with one gluon exchange (via $\mathcal{W}_{\mathcal{R}}^{(2)}$, obtained by evaluating the Wilson line diagrams in figure 2) and then exponentiating in order to resum length-enhanced diagrams as we have just discussed. From our approach, we know that eq. (2.15) could be extended to higher orders in the coupling by including further disconnected diagrams in (2.14). Analogously, the calculation in appendix D can immediately be generalized to analyze a Boltzmann equation with more terms in its collision kernel, and to show that if the collision kernel includes all terms to arbitrarily high orders in the coupling the result of the Boltzmann equation approach would indeed agree with our more general expression (2.14). 
The expression for $\mathcal{W}_{\mathcal{R}}^{(2)}$ is given in (2.12) as a function of the gluon propagator in coordinate space. Together with (2.12), the result (2.15) provides an expression for the probability distribution that describes transverse momentum broadening in a weakly coupled plasma of any length $L$, thick or thin. In appendix B we complete the analysis of a thin medium, where $g^{2} C_{\mathcal{R}} L T \ll 1$, disconnected diagrams are not length-enhanced, and only the diagrams in figure 2 contribute.

We end this section by rewriting the expression $(2.12)$ for $\mathcal{W}_{\mathcal{R}}^{(2)}$, which appears in the final result (2.15), in Fourier space. We first introduce the Wightman propagator in momentum space through

$$
D^{>}(X)=\int \frac{d^{4} Q}{(2 \pi)^{4}} e^{-i Q \cdot X} D^{>}(Q) .
$$

Here and below, we denote Lorentz four-vectors by an uppercase character, e.g. $Q$, and the modulus of the three-vector by lowercase character, e.g. $q$. Integrating over $y^{-}$in $(2.12)$ (taking $L \rightarrow \infty$ ) yields a delta function $\delta\left(q^{+}\right)$. Keeping in mind that the coordinate-space gluon fields are evaluated on the negative light-cone $y^{+}=0$, we then find that

$$
\mathcal{W}_{\mathcal{R}}^{(2)}\left(x_{\perp}\right)=-g^{2} C_{\mathcal{R}} L^{-} \times \int \frac{d q^{-} d^{2} q_{\perp}}{(2 \pi)^{3}}\left[1-e^{i q_{\perp} \cdot x_{\perp}}\right] D^{>}\left(q^{-}, q_{\perp}\right) .
$$

Finally, the propagator $D^{>}$can be written as [70]

$$
D^{>}(Q)=\left[1+f\left(q^{0}\right)\right] 2 \operatorname{Re} D_{R}(Q)
$$

where $f\left(q^{0}\right)$ is the Bose-Einstein distribution function and $D_{R}(Q)$ is the retarded propagator. We see that in a weakly coupled plasma $P\left(k_{\perp}\right)$ depends only on the retarded gluon propagator. Our goal in the next section will be to derive an explicit expression for the retarded propagator $D_{R}(Q)$, from which $D^{>}(Q)$ can be obtained using $(2.19), \mathcal{W}_{\mathcal{R}}^{(2)}$ can then be obtained using (2.18), and the probability distribution describing momentum broadening in a weakly coupled QCD plasma then follows using (2.15).

\section{$3 \quad$ Retarded gluon propagator}

In this section, we evaluate the real time expression for the retarded gluon propagator using the Schwinger-Keldysh formalism, with the two long light-like segments of the contour separated infinitesimally in the imaginary time direction. We give a brief review of the real-time field theory framework that we use in appendix A. The retarded gluon propagator $D_{R \mu \nu}$ is obtained by solving the Dyson equation

$$
D_{R \mu \nu}^{-1}(Q)=\left(D_{R \mu \nu}^{\mathrm{free}}(Q)\right)^{-1}+i \Pi_{R \mu \nu}(Q),
$$

where $D_{R \mu \nu}^{\mathrm{free}}$ is the free retarded propagator and $\Pi_{R \mu \nu}$ is the retarded self-energy. In a generic covariant gauge, the former reads

$$
\left(D_{R \mu \nu}^{\mathrm{free}}(Q)\right)^{-1}=i Q^{2}\left[g_{\mu \nu}-\left(1-\frac{1}{\xi}\right) \frac{Q_{\mu} Q_{\nu}}{Q^{2}}\right]
$$



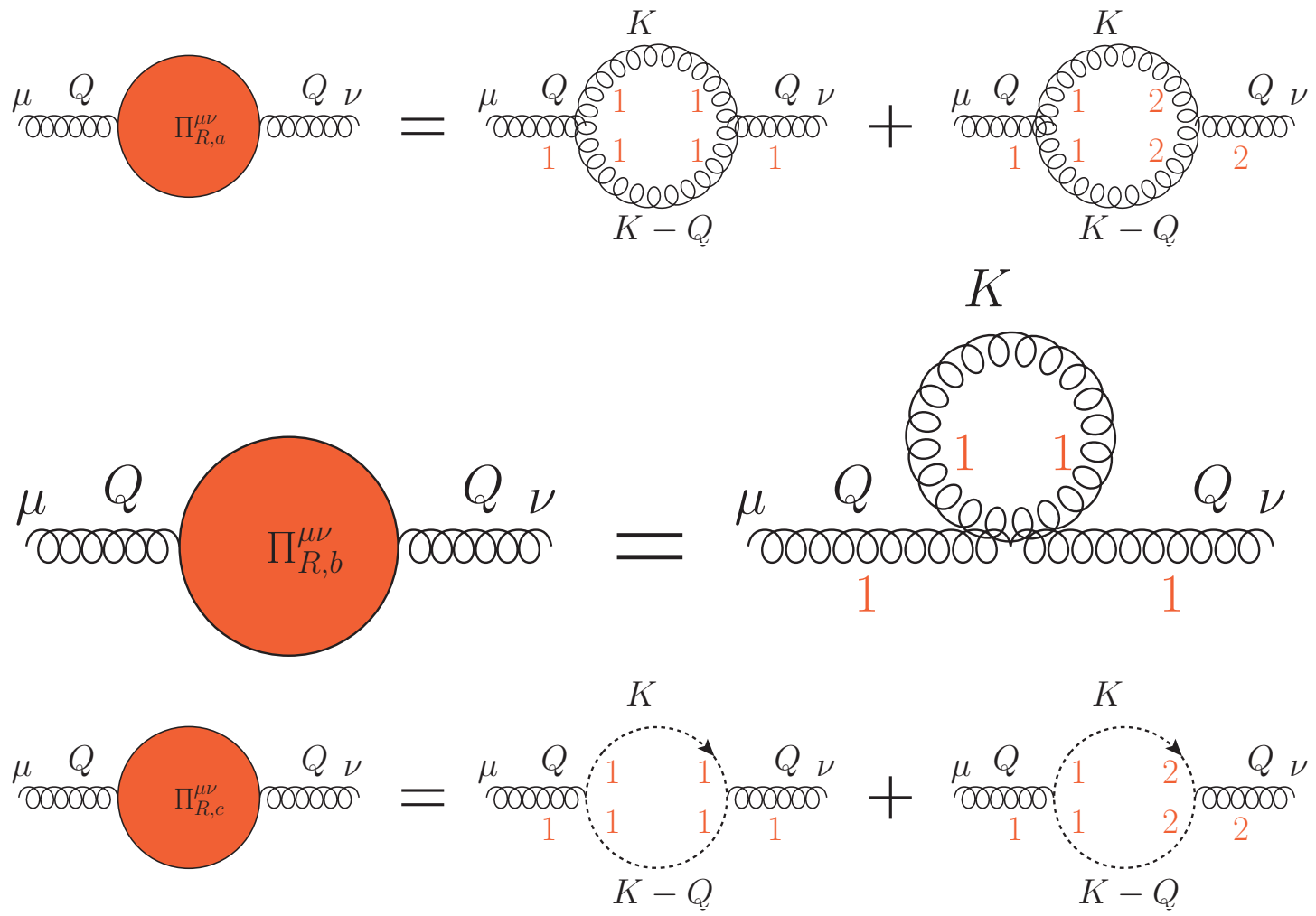

Figure 5. Diagrams contributing to $\Pi_{R \text {, YM }}^{\mu \nu}$, the contribution to the retarded self-energy from the Yang-Mills sector. The red numbers denote entries of the Schwinger-Keldysh matrix propagator.

where $\xi$ is the gauge fixing parameter. In this section, we compute the one-loop expression for the retarded self-energy. With the self-energy in hand we can solve (3.1) and extract the ++ component of the retarded propagator, which is what we need in order to determine the probability distribution $P\left(k_{\perp}\right)$ using the formalism that we set up in the previous section.

Unlike at zero temperature, the medium breaks Lorentz invariance and the self-energy tensor therefore has four independent components, in principle. We work in Feynman gauge $(\xi=1)$, where the one-loop self-energy is transverse [71-74], $Q^{\mu} \Pi_{R \mu \nu}(Q)=0$. In this case we only have two independent components:

$$
\Pi_{R \mu \nu}(Q)=\Pi_{R}^{T}(Q) P_{\mu \nu}^{T}+\Pi_{R}^{L}(Q) P_{\mu \nu}^{L} \quad(\xi=1),
$$

where the projectors $P_{\mu \nu}^{T}$ and $P_{\mu \nu}^{L}$ are defined in appendix E. After we substitute the expressions (3.2) and (3.3) into (3.1), we can invert the Dyson equation, obtaining

$$
D_{R \mu \nu}(Q)=\frac{i P_{\mu \nu}^{T}}{Q^{2}-\Pi_{R}^{T}(Q)}+\frac{i P_{\mu \nu}^{L}}{Q^{2}-\Pi_{R}^{L}(Q)}-\frac{i K_{\mu \nu}}{Q^{2}},
$$

where the projector $K_{\mu \nu}$ is also given in appendix E.

In what follows, we explicitly evaluate the full retarded gluon self-energy tensor $\Pi_{R \mu \nu}(Q)$ at one-loop, and then extract the components $\Pi_{R}^{T}(Q)$ and $\Pi_{R}^{L}(Q)$. We use the Schwinger-Keldysh formalism presented in appendix A, with the identification in (A.11) 


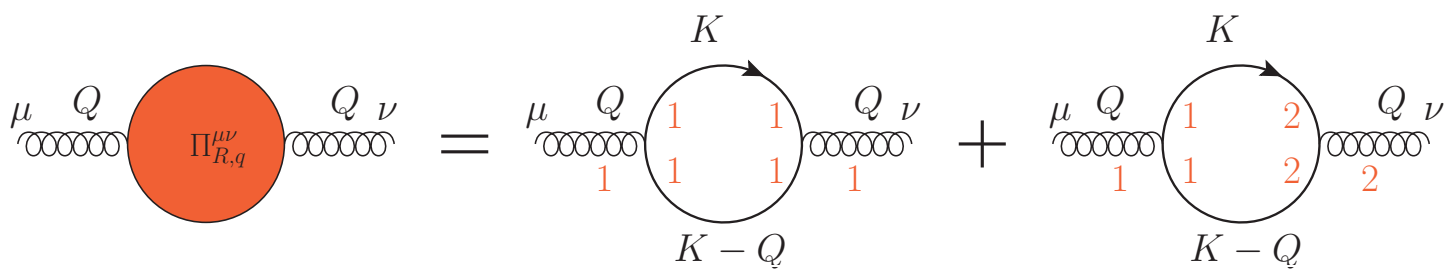

Figure 6. Diagrams contributing to $\Pi_{R \text {, quarks }}^{\mu \nu}$. Notation as in figure 5 .

between the retarded self-energy and the components of the self-energy matrix in the Schwinger-Keldysh formalism. There are two main contributions to $\Pi_{R \mu \nu}(Q)$, the YangMills sector and the quarks, so we write

$$
\Pi_{R}^{\mu \nu}=\Pi_{R, \mathrm{YM}}^{\mu \nu}+\Pi_{R, \text { quarks }}^{\mu \nu} .
$$

In the Yang-Mills sector we have three contributions

$$
\Pi_{R, \mathrm{YM}}^{\mu \nu}=\Pi_{R, a}^{\mu \nu}+\Pi_{R, b}^{\mu \nu}+\Pi_{R, c}^{\mu \nu},
$$

corresponding to the different diagrams shown in figure 5: a) gluon loop with the three-point vertex; b) gluon loop with the four-point vertex; c) ghost loop. We start from the diagrams for $\Pi_{R, a}^{\mu \nu}$ in figure 5 and use the standard Feynman rules for the three-gluon vertex to obtain the expression

$$
\begin{aligned}
\Pi_{R, a}^{\mu \nu}= & \frac{i}{4} g^{2} N_{c} \int \frac{d^{4} K}{(2 \pi)^{4}}\left[D_{R}(K) D_{S}(K-Q)+D_{S}(K) D_{A}(K-Q)\right] \\
& \times\left[-g^{\mu \nu}\left(5 Q^{2}+2 K^{2}-2 K \cdot Q\right)+2 Q^{\mu} Q^{\nu}+5 Q^{\mu} K^{\nu}+5 K^{\mu} Q^{\nu}-10 K^{\mu} K^{\nu}\right] .
\end{aligned}
$$

The explicit expressions for the retarded, advanced and symmetric propagators $D_{R}, D_{A}$ and $D_{S}$ are given in appendix A. We can combine the first two terms on the right-hand side of (3.7) by changing the loop integration variable $K \rightarrow Q-K$ in one of them. The vertex factor is left unchanged, whereas for the propagators we have

$$
D_{R}(K) D_{S}(K-Q) \rightarrow D_{S}(K) D_{A}(K-Q) .
$$

The two contributions are then identical, and we obtain

$$
\begin{aligned}
\Pi_{R, a}^{\mu \nu}= & \frac{i}{2} g^{2} N_{c} \int \frac{d^{4} K}{(2 \pi)^{4}} D_{S}(K) D_{A}(K-Q) \\
& \times\left[-g^{\mu \nu}\left(5 Q^{2}+2 K^{2}-2 K \cdot Q\right)+2 Q^{\mu} Q^{\nu}+5 Q^{\mu} K^{\nu}+5 K^{\mu} Q^{\nu}-10 K^{\mu} K^{\nu}\right] .
\end{aligned}
$$

Next we consider the contribution $\Pi_{R, b}^{\mu \nu}$. In this case we have only one diagram, since interaction vertices cannot connect fields on different segments of the Schwinger-Keldysh contour, and it reads

$$
\Pi_{R, b}^{\mu \nu}=g^{2} N_{c} \int \frac{d^{4} K}{(2 \pi)^{3}} \delta\left(K^{2}\right) n_{B}\left(k_{0}\right)\left(-3 g^{\mu \nu}\right) .
$$


Finally, since we are working in the Feynman gauge, there is also a contribution $\Pi_{R, c}^{\mu \nu}$ from ghosts in the loop. Its calculation is very similar to the one for the first contribution $\Pi_{R, a}^{\mu \nu}$, and in particular the two sub-diagrams in figure 6 are combined together by the same shift (3.8). After some algebra, the only difference with respect to the previous case is the interaction vertex, and we have

$$
\Pi_{R, c}^{\mu \nu}=i g^{2} N_{c} \int \frac{d^{4} K}{(2 \pi)^{4}} D_{S}(K) D_{A}(K-Q)\left[K^{\mu} K^{\nu}-Q^{\nu} K^{\mu}\right] .
$$

Upon summing (3.9), (3.10) and (3.11), we find that the full gauge contribution to the retarded gluon self-energy is given by

$$
\begin{aligned}
\Pi_{R, \mathrm{YM}}^{\mu \nu}= & g^{2} N_{c} \int \frac{d^{4} K}{(2 \pi)^{3}} \delta\left(K^{2}\right) n_{B}\left(k_{0}\right) \frac{1}{(Q-K)^{2}-i \operatorname{sgn}\left(k_{0}-q_{0}\right) \epsilon} \times \\
& \times\left[g^{\mu \nu}\left(2 Q^{2}+4 Q \cdot K-K^{2}\right)-2 Q^{\mu} Q^{\nu}-6 Q^{\mu} K^{\nu}-2 K^{\mu} Q^{\nu}+8 K^{\mu} K^{\nu}\right] .
\end{aligned}
$$

We assume that there are $N_{f}$ quarks in the theory. The fermion loop contribution is shown in figure 6 , and its calculation proceeds analogously to that for the gauge contribution. We find

$$
\begin{aligned}
\Pi_{R, \text { quarks }}^{\mu \nu}= & 4 g^{2} N_{f} \int \frac{d^{4} K}{(2 \pi)^{3}} \delta\left(K^{2}\right) n_{F}\left(k_{0}\right) \frac{1}{(Q-K)^{2}-i \operatorname{sgn}\left(k_{0}-q_{0}\right) \epsilon} \times \\
& \times\left[g^{\mu \nu}\left(Q \cdot K-K^{2}\right)-Q^{\mu} K^{\nu}-K^{\mu} Q^{\nu}+2 K^{\mu} K^{\nu}\right] .
\end{aligned}
$$

The full retarded self-energy defined in (3.5) is given by the sum of the two results (3.12) and (3.13). We use the transverse and longitudinal projectors defined in appendix E to extract its components $\Pi_{R}^{T}(Q)$ and $\Pi_{R}^{L}(Q)$, defined by (3.3), in order to evaluate the expression for the retarded gluon propagator in (3.4). The longitudinal component is projected out as follows

$$
\Pi_{R}^{L}=P_{L \nu \mu} \Pi_{R}^{\mu \nu}=-\frac{U_{\mu} U_{\nu}}{N^{2}} \Pi_{R}^{\mu \nu}=\frac{Q^{2}}{q^{2}} \Pi_{R}^{00},
$$

and we get

$$
\begin{aligned}
\Pi_{R, \mathrm{YM}}^{L} & =\frac{Q^{2}}{q^{2}} g^{2} N_{c} \int \frac{d^{4} K}{(2 \pi)^{3}} \delta\left(K^{2}\right) n_{B}\left(k_{0}\right) \frac{2 Q^{2}+4 Q \cdot K-2 q_{0}^{2}-8 q_{0} k_{0}+8 k_{0}^{2}}{(Q-K)^{2}-i \operatorname{sgn}\left(k_{0}-q_{0}\right) \epsilon}, \\
\Pi_{R, \text { quarks }}^{L} & =\frac{4 Q^{2}}{q^{2}} g^{2} N_{f} \int \frac{d^{4} K}{(2 \pi)^{3}} \delta\left(K^{2}\right) n_{F}\left(k_{0}\right) \frac{k_{0}^{2}+k^{2}-2 q_{0} k_{0}+Q \cdot K}{(Q-K)^{2}-i \operatorname{sgn}\left(k_{0}-q_{0}\right) \epsilon} .
\end{aligned}
$$

Likewise, the transverse component reads

$$
\begin{aligned}
& \Pi_{R}^{T}=\frac{1}{2} P_{T \nu \mu} \Pi_{R}^{\mu \nu}=-\frac{1}{2}\left[\Pi_{R}+\Pi_{R}^{L}\right], \\
& \Pi_{R} \equiv g_{\mu \nu} \Pi_{R}^{\mu \nu},
\end{aligned}
$$

where the factor of $1 / 2$ arises because the projector $P_{T}$ defined in appendix $\mathrm{E}$ has trace -2 . In the second equality, we have defined the trace of the retarded self-energy $\Pi_{R}$ and we have also identified the longitudinal self-energy just found above. Thus, once we know the 
longitudinal component, in order to get the transverse component we need only compute the trace of the self-energy and can then use (3.16). We find that the two contributions to the trace are given by

$$
\begin{aligned}
\Pi_{R, \mathrm{YM}} & =g^{2} N_{c} \int \frac{d^{4} K}{(2 \pi)^{3}} \delta\left(K^{2}\right) n_{B}\left(k_{0}\right) \frac{6 Q^{2}+8 Q \cdot K+4 K^{2}}{(Q-K)^{2}-i \operatorname{sgn}\left(k_{0}-q_{0}\right) \epsilon}, \\
\Pi_{R, \text { quarks }} & =g^{2} N_{f} \int \frac{d^{4} K}{(2 \pi)^{3}} \delta\left(K^{2}\right) n_{F}\left(k_{0}\right) \frac{8 Q \cdot K-2 K^{2}}{(Q-K)^{2}-i \operatorname{sgn}\left(k_{0}-q_{0}\right) \epsilon},
\end{aligned}
$$

for the pure gauge and quark contributions, respectively.

In order to obtain an explicit expression for the retarded gluon propagator of eq. (3.4), we need the longitudinal self-energy given in (3.14), and the transverse component obtained by combining (3.16) and (3.17). The expressions we have obtained so far are valid for any value of the gluon external momentum $Q$. However, as we have shown in (2.18), we only need the retarded gluon propagator evaluated on the negative light-cone, namely for $q^{+}=0$. For nonzero transverse momentum $q_{\perp}$ this corresponds to space-like external momentum $Q^{2}=-q_{\perp}^{2}<0$, in which case the self-energy has a non-vanishing imaginary part. This is crucial to our analysis, since what enters the calculation of $P\left(k_{\perp}\right)$ is the real part of the retarded propagator, as shown in eqs. (2.12), (2.15) and (2.19). The retarded propagator in (3.4) has a real part if and only if the self-energy has an imaginary part. Without this imaginary part, the probability distribution $P\left(k_{\perp}\right)$ in $(2.15)$ would just be a delta function centered at $k_{\perp}=0$, and thus we would not have any momentum broadening.

In what follows, we sketch the extraction of the longitudinal component of the selfenergy arising from loops involving gauge bosons and ghosts, and just state the final result for the transverse component. Starting from the explicit expression (3.15), we first integrate over $k_{0}$, imposing the on-shell condition for the loop momentum via the delta function. We get two different contributions, for $k_{0}=k$ and $k_{0}=-k$. The integration over the spatial components of the loop momentum is performed in polar coordinates, with the polar axis defined by the direction of the spatial component $\vec{q}$ of the external momentum. The integration over the azimuthal angle $\phi$ is straightforward, giving just a $2 \pi$ factor. The polar angle $\theta$ satisfies $\cos \theta=\vec{q} \cdot \vec{k}$, and after we integrate over it we find

$$
\begin{aligned}
\Pi_{R, \mathrm{YM}}^{L}= & \frac{g^{2} N_{c} T^{2}}{6} \frac{q_{\perp}^{2}}{q^{2}}+\frac{g^{2} N_{c}}{8 \pi^{2}} \frac{q_{\perp}^{2}}{q^{3}} \int_{0}^{\infty} d k n_{B}(k) \\
& \times\left[\left(2 q^{2}-\left(2 k-q_{0}\right)^{2}\right) \log \left(\frac{q_{\perp}^{2}+2 k\left(q_{0}-q\right)+i \epsilon \operatorname{sgn}\left(k-q_{0}\right)}{q_{\perp}^{2}+2 k\left(q_{0}+q\right)+i \epsilon \operatorname{sgn}\left(k-q_{0}\right)}\right)+\left(\begin{array}{c}
q_{0} \rightarrow-q_{0} \\
\epsilon \rightarrow-\epsilon
\end{array}\right)\right]
\end{aligned}
$$

where we have used $\int_{0}^{\infty} d k k n_{B}(k)=\frac{\pi^{2} T^{2}}{6}$. The logarithms appearing in the expression (3.18) develop an imaginary part for $\left(q_{\perp}^{2} \pm 2 k q_{0}\right)^{2}<(2 k q)^{2}$. We expand the logarithms in the $\epsilon \rightarrow 0$ limit, obtaining a logarithm of the absolute value and a Heaviside step function for the real and imaginary part, respectively. We then identify the real and imaginary 
part of the longitudinal self-energy, obtaining

$$
\begin{aligned}
\operatorname{Re} \Pi_{R, \mathrm{YM}}^{L}= & \frac{g^{2} N_{c} T^{2}}{6} \frac{q_{\perp}^{2}}{q^{2}}+\frac{g^{2} N_{c}}{8 \pi^{2}} \frac{q_{\perp}^{2}}{q^{3}} \int_{0}^{\infty} d k n_{B}(k) \\
& \times\left[\left(2 q^{2}-\left(2 k-q_{0}\right)^{2}\right) \log \left|\frac{q_{\perp}^{2}+2 k\left(q_{0}-q\right)}{q_{\perp}^{2}+2 k\left(q_{0}+q\right)}\right|+\left(q_{0} \rightarrow-q_{0}\right)\right], \\
\operatorname{Im} \Pi_{R, \mathrm{YM}}^{L}= & \frac{g^{2} N_{c}}{8 \pi} \frac{q_{\perp}^{2}}{q^{3}}\left[\int_{\frac{q_{0}+q}{2}}^{\infty} d k n_{B}(k)\left(2 q^{2}-\left(2 k-q_{0}\right)^{2}\right)-\left(q_{0} \rightarrow-q_{0}\right)\right] .
\end{aligned}
$$

The only integrations which are left are over the magnitude of the loop three-momentum. The integral for the real part can only be evaluated numerically, whereas the one for the imaginary part can be expressed in terms of the polylogarithmic functions $\operatorname{Li}_{\nu}(z)$. The expressions for the real and imaginary part of the longitudinal self-energy coming from quark loop are evaluated analogously, with the main difference being the appearance of the Fermi-Dirac distribution thermal distribution function instead of the Bose-Einstein. After combining the Yang-Mills piece and the contribution from $N_{f}$ quarks we obtain

$$
\begin{aligned}
\operatorname{Re} \Pi_{R}^{L}= & \left(N_{c}+\frac{N_{f}}{2}\right) \frac{g^{2} T^{2}}{6} \frac{q_{\perp}^{2}}{q^{2}}+\frac{g^{2}}{8 \pi^{2}} \frac{q_{\perp}^{2}}{q^{3}}\left[\int_{0}^{\infty} d k \log \left|\frac{q_{\perp}^{2}+2 k\left(q_{0}-q\right)}{q_{\perp}^{2}+2 k\left(q_{0}+q\right)}\right|\right. \\
& \left.\times\left[N_{c} n_{B}(k) q^{2}-\left(N_{f} n_{F}(k)+N_{c} n_{B}(k)\right)\left(4 k^{2}-4 k q_{0}-q_{\perp}^{2}\right)\right]+\left(q_{0} \rightarrow-q_{0}\right)\right], \\
\operatorname{Im} \Pi_{R}^{L}= & \frac{g^{2} N_{c}}{24 \pi} \frac{q_{\perp}^{2}}{q^{3}}\left[5 q_{0}^{3}+8 q_{0} T^{2} \pi^{2}+6 q_{0} q_{\perp}^{2}\right] \\
& -\frac{g^{2} T}{4 \pi} \frac{q_{\perp}^{2}}{q^{2}}\left[\frac{N_{f}}{2}\left(T \operatorname{Li}_{2}\left(-e^{\frac{q_{0}-q}{2 T}}\right)+\frac{2 T^{2}}{q} \operatorname{Li}_{3}\left(-e^{\frac{q_{0}-q}{2 T}}\right)\right)+\right. \\
& \left.-N_{c}\left(\frac{q}{2} \log \left(1-e^{\frac{q-q_{0}}{2 T}}\right)-2 T \operatorname{Li}_{2}\left(e^{\frac{q-q_{0}}{2 T}}\right)+\frac{4 T^{2}}{q} \operatorname{Li}_{3}\left(e^{\frac{q-q_{0}}{2 T}}\right)\right)-\left(q_{0} \rightarrow-q_{0}\right)\right],
\end{aligned}
$$

By similar means, we calculate the trace of self-energy tensor (3.17) which we then combine with the longitudinal components in (3.20) to obtain the transverse self-energy according to eq. (3.16). We find

$$
\begin{aligned}
\operatorname{Re} \Pi_{R}^{T}= & \left(N_{c}+\frac{N_{f}}{2}\right) \frac{g^{2} T^{2}}{12}\left(1+\frac{q_{0}^{2}}{q^{2}}\right)+\frac{g^{2} q_{\perp}^{2}}{16 \pi^{2} q^{3}}\left[\int_{0}^{\infty} d k \log \left|\frac{q_{\perp}^{2}+2 k\left(q_{0}-q\right)}{q_{\perp}^{2}+2 k\left(q_{0}+q\right)}\right|\right. \\
& \left.\times\left[2 N_{c} n_{B}(k) q^{2}+\left(N_{c} n_{B}(k)+N_{f} n_{F}(k)\right)\left(q^{2}+\left(2 k-q_{0}\right)^{2}\right)\right]+\left(q_{0} \rightarrow-q_{0}\right)\right], \\
\operatorname{Im} \Pi_{R}^{T}= & \frac{g^{2} N_{c}}{48 \pi} \frac{q_{\perp}^{2}}{q^{3}}\left[10 q_{0}^{3}-8 q_{0} T^{2} \pi^{2}+9 q_{0} q_{\perp}^{2}\right]+ \\
& +\frac{g^{2} T}{4 \pi} \frac{q_{\perp}^{2}}{q^{3}}\left[-\frac{N_{f}}{2}\left(q^{2} \log \left(1+e^{\frac{q_{0}-q}{2 T}}\right)+2 q T \operatorname{Li}_{2}\left(-e^{\frac{q_{0}-q}{2 T}}\right)+4 T^{2} \operatorname{Li}_{3}\left(-e^{\frac{q_{0}-q}{2 T}}\right)\right)+\right. \\
& \left.+N_{c}\left(q^{2} \log \left(1-e^{\frac{q-q_{0}}{2 T}}\right)-q T \operatorname{Li}_{2}\left(e^{\frac{q-q_{0}}{2 T}}\right)-2 T^{2} \operatorname{Li}_{3}\left(e^{\frac{q-q_{0}}{2 T}}\right)\right)-\left(q_{0} \rightarrow-q_{0}\right)\right] .
\end{aligned}
$$

Thus, we have obtained the components of the gluon self-energy in (3.20) and (3.21) by direct calculation in real-time field theory. In the imaginary time formalism, the gluon self-energies were first computed in refs. [71, 75]. 
We end this section by performing a check of our calculation, a check whose results we shall use in section 4. We recover the HTL self-energies [61-65], which are valid for external momenta of order $g T$ or below. In this regime, the main contribution to the loop integral comes from hard loop momenta $k \sim T$, so the procedure corresponds to expanding the integrand in powers of $q / k$. Both the real and imaginary parts of the gluon self-energy that we have obtained above can be computed analytically to leading order in this expansion, resulting in

$$
\begin{aligned}
\left.\operatorname{Re} \Pi_{R}^{L}\right|_{H T L} & =\frac{m_{D}^{2} q_{\perp}^{2}}{q^{2}}\left(1+\frac{q_{0}}{2 q} \log \left(\frac{q-q_{0}}{q+q_{0}}\right)\right), \\
\left.\operatorname{Im} \Pi_{R}^{L}\right|_{H T L} & =\pi m_{D}^{2}\left(\frac{q_{\perp}^{2} q_{0}}{2 q^{3}}\right), \\
\left.\Pi_{R}^{T}\right|_{H T L} & =\frac{m_{D}^{2}-\left.\Pi_{R}^{L}\right|_{H T L}}{2},
\end{aligned}
$$

where $m_{D}^{2}$ is the Debye mass squared

$$
m_{D}^{2}=\frac{g^{2} T^{2}}{3}\left(N_{c}+\frac{N_{f}}{2}\right) .
$$

As we will show explicitly in section 4, perturbation theory breaks down in the region where the external momentum in a gluon propagator is of order $g^{2} T$. We will fix this problem by using the HTL self-energies given in eq. (3.22), which are well-behaved in the region where ordinary perturbation theory becomes problematic.

\section{Breakdown of perturbation theory and self-energy matching}

The purpose of this work is to evaluate the probability distribution $P\left(k_{\perp}\right)$ in $(2.15)$. In order to do that we have to evaluate the gluon propagator for $q^{+}=0$, and integrate it over $d q^{-} d^{2} q_{\perp}$, as in eq. (2.18). In particular, we have to integrate the gluon propagator over the region in momentum space where both $q^{-}$and $q_{\perp}$ are of order $g^{2} T$ or smaller. We shall begin this section with an explicit demonstration of the breakdown of perturbation theory at the scale $g^{2} T$ in our self-energy results (3.20) and (3.21), and then describe how we shall evade this difficulty. This problem in finite temperature non-abelian gauge theory has been known for many years, since the early work of refs. [59, 60]. Here, we focus on the pure Yang-Mills contribution to the self-energy (setting $N_{f}=0$ ), since the matter fermions are not responsible for the breakdown of perturbation theory in the infrared. We only consider the real part of the self-energies, since that is where the problem arises.

We can find the infrared breakdown of perturbation theory that occurs where both $q^{-}$and $q_{\perp}$ are of order $g^{2} T$ by focussing on the slice through this region where $q_{0}=0$ and $0<q_{\perp}<g^{2} T$. No problems arise in the longitudinal self-energy: it is gauge independent [60] and, upon taking the appropriate limit in (3.20), we find

$$
\operatorname{Re} \Pi_{R, Y M}^{L}\left(q_{0}=0, q_{\perp} \rightarrow 0\right) \rightarrow m_{D}^{2}=\frac{1}{3} g^{2} N_{c} T^{2} .
$$


This longitudinal self-energy is responsible for screening the electric modes, giving them a screening mass $m_{\mathrm{el}}^{2}=g^{2} N_{c} T^{2} / 3$. It does not cause any problems for perturbation theory. The problems arise in the transverse self-energy (3.21). In order to extract the infrared limit, we divide the loop integral $d k$ in (3.21) into hard and soft regions. When $q_{0}=0$, $q_{\perp}<g^{2} T$, and the loop integration variable is hard $\left(k \gtrsim T\right.$ and therefore $\left.k \gg g^{2} T\right)$ we find that the integrand in the expression (3.21) for $\Pi_{R, Y M}^{T}$ vanishes. We return to this point below but, first, we push ahead into trouble by attempting to evaluate the contribution to $\Pi_{R, Y M}^{T}$ from the region of the $d k$ integral in (3.21) where $k \ll T$. Here we are allowed to use the "soft approximation" $\left(n_{B}(k) \sim T / k\right)$ in $(3.21)$. We find ${ }^{4}$

$$
\operatorname{Re} \Pi_{R, Y M}^{T}\left(q_{0}=0, q_{\perp} \rightarrow 0\right) \rightarrow \frac{3}{8 \pi^{2}} g^{2} N_{c} q_{\perp} T \int_{0}^{\infty} \frac{d k}{k} \log \left|\frac{q_{\perp}-2 k}{q_{\perp}+2 k}\right|=-\frac{3}{16} g^{2} T N_{c} q_{\perp} .
$$

We immediately notice that for external momentum $q_{\perp} \sim g^{2} T$, the real part of the transverse self-energy $\operatorname{Re} \Pi_{R, Y M}^{T}$ is comparable to $q_{\perp}^{2}$. This introduces an unphysical pole at a space-like momentum of order $g^{2} T$ in the propagator (3.4). It also invalidates the perturbative expansion of the propagator (3.4), in which $\Pi_{R}$ is supposed to be subleading compared to $Q^{2}$. Clearly, perturbation theory cannot be trusted anymore at and below the scale $g^{2} T$, and neither can the result (4.2).

It is expected that a magnetic mass of order $g^{2} T$ arises from nonperturbative effects.

Even if this happens, though, perturbation theory still breaks down at the $g^{6}$ order, as shown in ref. [59] by an explicit example. (In contrast, neither perturbative nor nonperturbative effects generate a magnetic mass in an abelian gauge theory [76]. The leading term for the transverse self-energy goes as $g^{2} q_{\perp}^{2}$, as can be checked from our fermion loop result, and for this reason perturbation theory does not break down in the infrared limit.)

To take care of this problem, we use the HTL self-energy (3.22) in the problematic region. In this approximation, the transverse component of the gluon self-energy is gauge independent $[77,78]$ and does not give rise to any additional pole at $q \neq 0$, so we do not run into any infrared problems. Along the $q_{0}=0$ slice that we analyzed above, the HTL self-energy is so well-behaved that it in fact vanishes, as we already saw above. Using the HTL self-energy in the $g^{2} T$ momentum region avoids all infrared problems and gives us a well-behaved result at the leading order to which we are working, but of course it does not incorporate the effects of the magnetic mass of order $g^{2} T$, which is generated only nonperturbatively and so is absent in the HTL self-energy (3.22). In the high-temperature limit of QCD, the nonperturbative physics at momenta of order $g^{2} T$ is described by matching to a dimensionally reduced long-wavelength effective theory which turns out to be just Euclidean 3-dimensional $\mathrm{SU}\left(N_{c}\right)$ gauge theory with the dimensionful coupling constant $g_{E}$ given by $g_{E}^{2}=g^{2} T$. Following up on a suggestion by Caron-Huot [68], Laine has very recently shown

\footnotetext{
${ }^{4}$ Our result is obtained in Feynman gauge $(\xi=1)$. For a general covariant gauge the infrared behavior of the self-energy was first analyzed in [60] using the imaginary time formalism, finding

$$
\operatorname{Re} \Pi_{R, Y M}^{T}\left(q_{0}=0, q_{\perp} \rightarrow 0\right) \rightarrow-\frac{8+(1+\xi)^{2}}{64} g^{2} T N_{c} q_{\perp},
$$

consistent with our result in the Feynman gauge. This contribution is gauge dependent, but it cannot be set to zero by any gauge choice.
} 


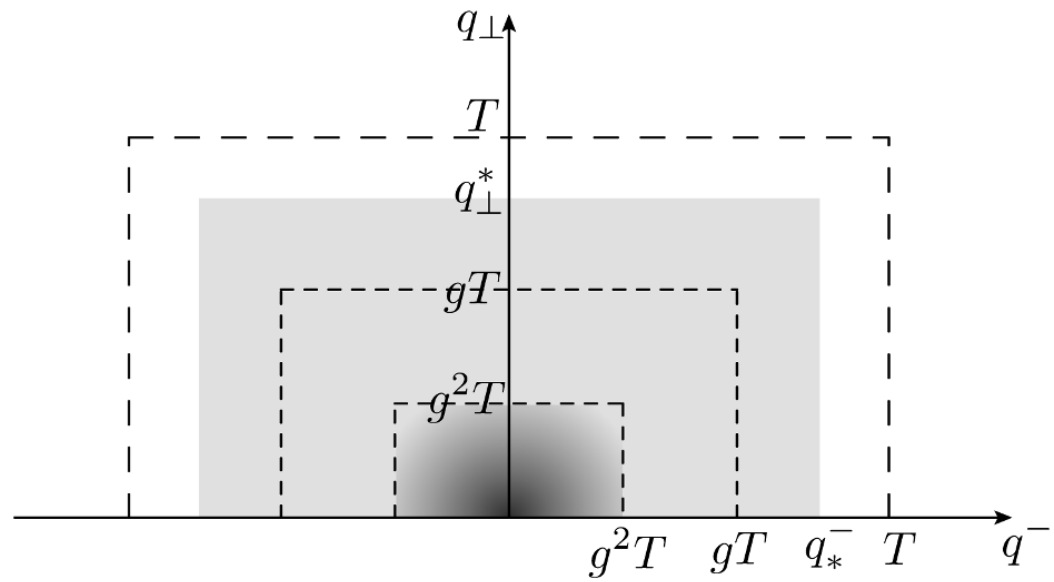

Figure 7. Self-energy matching illustration. In the momentum region shaded in grey, where $\left|q^{-}\right|<\left|q_{*}^{-}\right|$and $q_{\perp}<q_{\perp}^{*}$, we use HTL self-energies in the integration in (2.18), whereas we use full self-energies elsewhere, in the white regions. The darker grey region, where momenta are $\mathcal{O}\left(g^{2} T\right)$ is the dangerous region where we must use HTL self-energies. We do not want to do the matching from grey to white anywhere near this darker region. The HTL self-energies are not valid once momenta are $\mathcal{O}(T)$, so the grey region must not extend this far. We have checked explicitly that for $g \ll 1$ the numerical result for (2.18) is insensitive to where we match from grey to white, as long as $g T<\left|q_{*}^{-}\right|<T$ and $g T<k_{\perp}^{*}<T$.

that the nonperturbative contributions to $P\left(k_{\perp}\right)$ can be related to the static potential in this effective theory and he and others $[57,79]$ have used this elegant observation to show that the nonperturbative contribution to $P\left(k_{\perp}\right)$ is suppressed parametrically, contributing to $\hat{q}$ (the second moment of $P\left(k_{\perp}\right)$ ) only at order $g^{6} T^{3}$, and is further suppressed by a numerically small prefactor [79]. This result justifies the neglect of these nonperturbative effects that is inherent in our use of the HTL self-energy at momenta of order $g^{2} T$.

Although using the HTL self-energy nicely eliminates the infrared problems in perturbation theory, we cannot simply use the HTL self-energy throughout our calculation because it is not valid for hard external momenta, which in our case corresponds to $q_{0} \sim T$ (and therefore $q^{-} \sim T$ ) and $q_{\perp} \sim T$ in (2.18). The correct procedure is then to use the full or HTL self-energies in the regimes where each is valid, and to match in a region where both are valid. The strategy is illustrated in figure 7 . The darkest shading illustrates the momentum scales of order $g^{2} T$ where we must use the HTL self energies because perturbation theory runs into troubles if we do not do so. In the regions where momenta are of order $T$, the HTL self energies are no longer valid and we have to use the full self energies. We must match from HTL to full self energies in a region in which both are valid. As illustrated in figure 7 , we perform the matching at $q_{*}^{-}$and $q_{\perp}^{*}$ such that $g T<q_{*}^{-}<T$ and $g T<q_{\perp}^{*}<T$. We find that the matching is smooth at weak coupling $g \ll 1$, with the exact location of the matching scales $q_{*}^{-}$and $q_{\perp}^{*}$ not affecting our final results as long as the matching is performed in the appropriate region.

Before presenting our results in the next section, we close this section by comparing our approach to perturbation theory, illustrated in the figure 7, to that in some previous field- 
theoretical analyses of momentum broadening in weakly coupled quark-gluon plasma [6669]. In the soft region $\left(k_{\perp}<T\right)$, these authors use the HTL approximation in their calculations of the probability for momentum broadening. In the notation of our eq. (2.18), using the HTL approximation is well justified when $q_{\perp}<T$ only over the regime of the $d q^{-}$ integration in which $\left|q^{-}\right|<T$, but not over the entire range of the $d q^{-}$integration. We have checked, however, that if we were to use the HTL self energies for $q_{\perp}<T$ over the entire $d q^{-}$integration the error introduced is quite small. Our results therefore agree with theirs in this momentum regime, for a thin medium. But, only for a thin medium because once the medium becomes thick one must resum $L$-enhanced diagrams, as we have done. In the hard region $\left(k_{\perp} \gg T\right)$ Arnold and Dogan correctly use the unscreened gluon propagator [66]. In this regime, resumming $L$-enhanced diagrams does not modify our results significantly (because it is more likely to pick up a very large $k_{\perp}$ from a single improbable hard kick than from several less hard but still improbable kicks) and our results therefore agree with those of ref. [66] for $k_{\perp} \gg T$. We have been quite careful about how we match from the hard region, including that at $\left|q^{-}\right| \gg T$ at small $q_{\perp}$, as we have described in figure 7 . This care is unnecessary when $k_{\perp} \gg T$, and when $k_{\perp}<T$ it turns out that doing the matching carefully as we do modifies our results less than the resummation of $L$-enhanced diagrams does. So, we shall see in the next section that our calculation correctly reproduces the results derived with other techniques where it should. However, when the medium is thick enough that the effects of the resummation that we have done become important, we find disagreements with previous results in the soft perpendicular momentum region.

\section{Results and discussion}

In sections II, III and IV we have presented a careful derivation of our expression for $P\left(k_{\perp}\right)$ in a weakly coupled plasma and a complete description of how we shall evaluate it. The derivation has turned out to be both subtle and technical at various points, and we therefore promised in section I that a reader not interested in subtleties or technical details could skip from the end of section I to here. For the benefit of such a reader, we begin here by restating the most salient points from the previous sections. After expanding the probability distribution for transverse momentum broadening (1.4) in the weak-coupling limit and after resumming an infinite class of "length-enhanced" diagrams that are important if $L$ is large enough that $g^{2} C_{\mathcal{R}} L T$ is not $\ll 1$, we found that transverse momentum broadening is described by

$$
P\left(k_{\perp}\right)=\int d^{2} x_{\perp} e^{-i k_{\perp} \cdot x_{\perp}} \exp \left[\mathcal{W}_{\mathcal{R}}^{(2)}\right] .
$$

The physical interpretation of resumming length enhanced diagrams is that doing includes the effect of multiple scattering; we show in appendix D that the same result (2.15) can equally well be derived by solving a Boltzmann equation for momentum broadening via multiple elastic collisions. In (2.15), the properties of the medium enter through

$$
\mathcal{W}_{\mathcal{R}}^{(2)}\left(x_{\perp}\right)=-g^{2} C_{\mathcal{R}} L^{-} \int \frac{d q^{-} d^{2} q_{\perp}}{(2 \pi)^{3}}\left[1-e^{i q_{\perp} \cdot x_{\perp}}\right] D^{>}\left(q^{-}, q_{\perp}\right)
$$


The Wightman gluon propagator $D^{>}$is directly related to the retarded gluon propagator by

$$
D^{>}(Q)=\left[1+f\left(q^{0}\right)\right] 2 \operatorname{Re} D_{R}(Q)
$$

where $f\left(q^{0}\right)$ is the Bose-Einstein distribution function and $D_{R}(Q)$ is given in (3.4) in terms of the self-energies that we have then computed explicitly in section 3. In section IV we explain where and why we use the full self energies or the HTL self energies in our evaluation of (3.4). The full and HTL self energies are given explicitly in eqs. (3.20), (3.21), (3.22).

\subsection{Results for a thin medium, and comparison to previous results}

Let us introduce the dimensionless variable

$$
\kappa \equiv \frac{g^{2} C_{R} L T}{2 \pi}
$$

proportional to the thickness of the medium $L$, which determines how important it is to resum $L$-enhanced diagrams. We begin by presenting our results for the case where $\kappa \ll 1$, meaning that there is no need to resum the $L$-enhanced diagrams at all. In this thin-medium regime, it is convenient to define the function

$$
P_{\text {thin }}\left(k_{\perp}\right) \equiv \frac{2 \sqrt{2} \pi \kappa}{T} \int \frac{d q^{-}}{2 \pi} D^{>}\left(q^{-}, k_{\perp}\right),
$$

because the resummed probability distribution (2.15) reduces to

$$
P\left(k_{\perp}\right)=P_{\text {thin }}\left(k_{\perp}\right) \quad \text { for } \quad k_{\perp} \neq 0 .
$$

This is shown explicitly in appendix B, where we also explain how to handle subtleties at $k_{\perp}=0$ correctly, so as to obtain a normalized probability distribution $P\left(k_{\perp}\right)$.

The correct IR and UV behavior of the probability distribution $P_{\text {thin }}\left(k_{\perp}\right)$ have each been obtained previously:

- In the IR region, Aurenche, Gelis and Zaraket showed by explicit calculation that (in our notation) [80]

$$
P_{\text {thin }}\left(k_{\perp}\right)=P_{\text {thin }}^{\mathrm{AGZ}}\left(k_{\perp}\right) \quad \text { for } \quad k_{\perp} \ll T
$$

where

$$
P_{\text {thin }}^{\mathrm{AGZ}}\left(k_{\perp}\right) \equiv \kappa \frac{2 \pi m_{D}^{2}}{k_{\perp}^{2}\left(k_{\perp}^{2}+m_{D}^{2}\right)},
$$

with the Debye mass squared as given in (3.23).

- In the UV region, $k_{\perp} \gg T$, the calculation of Arnold and Dogan shows that (again in our notation) [66]

$$
P_{\text {thin }}\left(k_{\perp}\right)=P_{\text {thin }}^{\mathrm{AD}}\left(k_{\perp}\right) \quad \text { for } \quad k_{\perp} \gg T
$$

where

$$
P_{\mathrm{thin}}^{\mathrm{AD}}\left(k_{\perp}\right)=\kappa\left(4 N_{c}+3 N_{f}\right) \frac{g^{2} \zeta(3) T^{2}}{\pi k_{\perp}^{4}},
$$

with $\zeta(3) \approx 1.202$ the Riemann zeta function. 
These expressions can each be obtained from our $P_{\text {thin }}\left(k_{\perp}\right)$, defined in eq. (5.2), by taking the IR or UV limits. To obtain the IR expression, we use the HTL self-energy everywhere, make the additional soft approximation, e.g. $n_{B}\left(q_{0}\right) \sim T / q_{0}$, and recover (5.5). To take the UV limit, we use the full self-energy rather than the HTL self-energy and keep only the first order solution to the Dyson equation (3.1), and recover (5.7). In figure 8 we plot $P_{\text {thin }}\left(k_{\perp}\right)$ multiplied by factors of $k_{\perp}^{3}$ and $k_{\perp}^{4}$, and show the agreement in the IR with the AGZ result and in the UV with the AD result. We see that at both $g=0.1$ and $g=0.3$, the agreement with the AGZ result is excellent, and extends to values of $k_{\perp} / m_{D}$ that are not small at all. In fact, for $g=0.01$ (which we have not plotted) this agreement extends beyond $k_{\perp}=10 m_{D}$. We also see that although at $g=0.3$ the matching described in section IV, see figure 7 , is smooth, at $g=1$ and $g=2$ it introduces a kink at $k_{\perp}=q_{\perp}^{*}$. This highlights the fact that a weak-coupling analysis is not quantitatively reliable at these larger values of $g$. There is a good reason for this: once $g \geq 1$, the separation of the scales $g^{2} T, g T$ and $T$ that we discussed in section 4 and used as depicted in figure 7 breaks down. In order to apply our calculation at $g=1$ and $g=2$, we need a prescription for how to do the matching described in figure 7 , even when the scales depicted are not separated. What we have done is to choose the matching scale on the horizontal axis of figure 7 as $q_{*}^{-}=T$, thinking it would be unreasonable to choose a larger $q_{*}^{-}$even if it is the case that $g T>T$. Then we have chosen the matching scale $q_{\perp}^{*}$ so as to make the probability distribution $P_{\text {thin }}\left(k_{\perp}\right)$ continuous at $k_{\perp}=q_{\perp}^{*}$, with a kink there but no discontinuity. Clearly we could instead have chosen a matching prescription at $g>1$ involving interpolation over a window in $k_{\perp}$, but this would have been no less arbitrary, given that there is a physical reason why the calculation is not under quantitative control at these large values of $g$.

For $g<1$, as discussed in section 4 the matching can be done anywhere in the range $g T<q_{\perp}^{*}, q_{*}^{-}<T$, because the HTL and full self-energies are in good agreement throughout this region. For $g=0.1$, the matching was done at $q_{*}^{-}=0.28 T$ and $q_{\perp}^{*}=0.3 T$ while for $g=0.3$ it was done at $q_{*}^{-}=0.42 T$ and $q_{\perp}^{*}=0.9 T$. We have checked that if we vary the locations at which the matching is done within the range between $g T$ and $T$, the correction to $P_{\text {thin }}\left(k_{\perp}\right)$ plotted in figure 8 is less than the thickness of the curves in the figure.

A leading order expression for $P_{\text {thin }}\left(k_{\perp}\right)$ for all $k_{\perp}$ was obtained in ref. [67] by interpolating between the small and large $k_{\perp}$ regimes. A next-to-leading-order result was derived in ref. [68], but within the HTL approximation. The HTL result of ref. [68] was then extended to the $k_{\perp}>T$ region by making the soft approximation discussed above. Another calculation of $P_{\text {thin }}$ valid in the IR can be found in ref. [69], where the momentum broadening distribution was obtained via a Langevin equation. The solution obtained there using the HTL self-energy reproduces the AGZ result.

\subsection{Complete results for the probability distribution $P\left(k_{\perp}\right)$}

The probability distribution $P\left(k_{\perp}\right)$ in $(1.4)$ is obtained by Fourier transforming the function $\exp \left[\mathcal{W}_{\mathcal{R}}^{(2)}\left(x_{\perp}\right)\right]$ with $\mathcal{W}_{\mathcal{R}}^{(2)}$ given by $(2.18)$. We see therefore that, if the medium being probed is a weakly-coupled plasma, $\mathcal{W}_{\mathcal{R}}^{(2)}\left(x_{\perp}\right)$ is the only "soft function" through which properties of the medium enter into the probability distribution for momentum broadening. $\mathcal{W}_{\mathcal{R}}^{(2)}\left(x_{\perp}\right)$ is proportional to $\kappa$ and depends on the gauge coupling constant $g$, with most of 


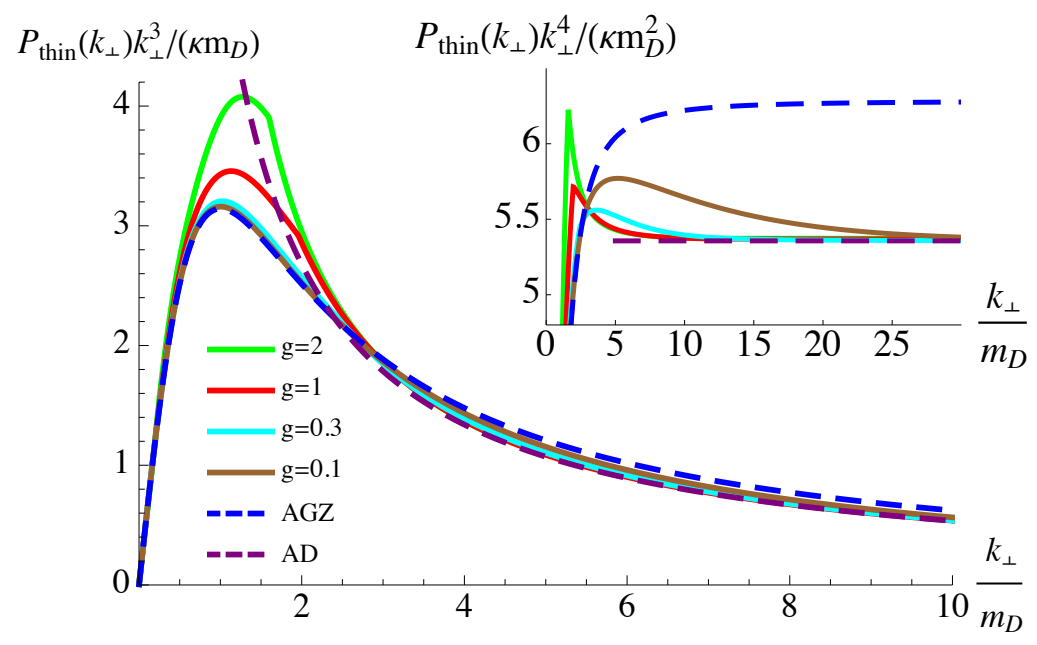

Figure 8. The continuous brown, light blue, red and green curves are the probability distribution $P_{\text {thin }}\left(k_{\perp}\right)$ for $g=0.1,0.3,1$ and 2 (bottom to top at low $k_{\perp}$, top to bottom at high $k_{\perp}$ ), multiplied by $k_{\perp}^{3}$ and $k_{\perp}^{4}$. In the IR, $P_{\text {thin }}\left(k_{\perp}\right)$ agrees with $P_{\text {thin }}^{\mathrm{AGZ}}\left(k_{\perp}\right)$ (shown as the dashed dark blue curves) and in the UV, $P_{\text {thin }}\left(k_{\perp}\right)$ agrees with $P_{\text {thin }}^{\mathrm{AD}}\left(k_{\perp}\right)$ (shown as the dashed purple curves). The only $L$-dependence in $P_{\text {thin }}$ arises from it being proportional to $\kappa$ meaning that, because we have plotted the probability distributions divided by $\kappa$, the quantities plotted are $L$-independent. We have scaled both axes by the appropriate power of the Debye mass $m_{D}$ to make the quantities plotted dimensionless. Scaling the plots in this way also ensures that $P_{\text {thin }}^{\mathrm{AGZ}}\left(k_{\perp}\right)$ and $P_{\text {thin }}^{\mathrm{AD}}\left(k_{\perp}\right)$, shown as the dashed curves, are independent of $g$. The kinks in the curves for $g=1$ and $g=2$ are located at the $k_{\perp}=q_{\perp}^{*}$ where we do the matching described in figure 7 .

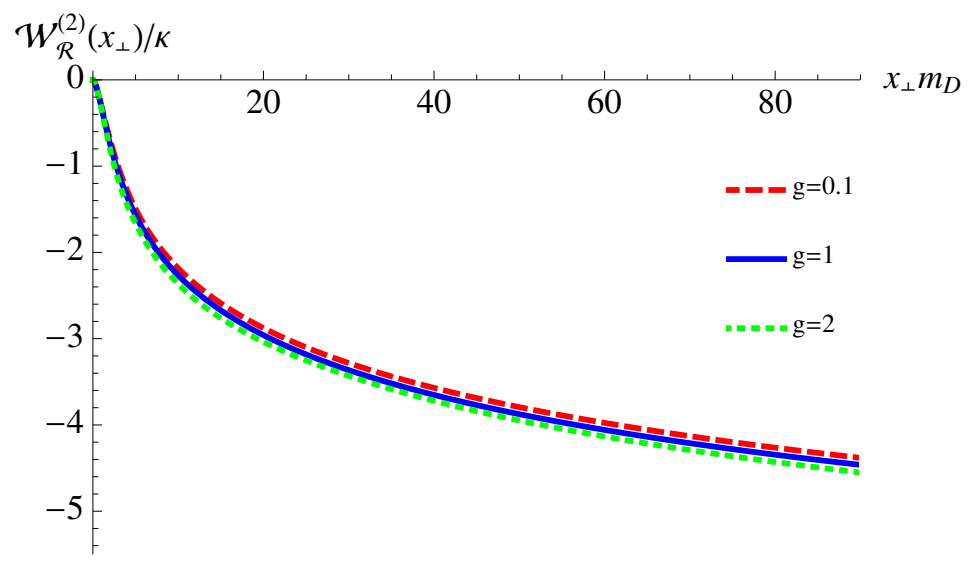

Figure 9. $\mathcal{W}_{\mathcal{R}}^{(2)}\left(x_{\perp}\right) / \kappa$ for gauge coupling constants $g=0.1,1$ and $2 . \mathcal{W}_{\mathcal{R}}^{(2)}\left(x_{\perp}\right) / \kappa$ is independent of $\kappa$ and, when plotted versus $x_{\perp}$ in units of the inverse Debye mass, is almost independent of $g$.

the latter dependence coming via its dependence on the Debye mass $m_{D}$ given in (3.23). We illustrate this in figure 9 , where we plot $\mathcal{W}_{\mathcal{R}}^{(2)}\left(x_{\perp}\right) / \kappa$ versus $x_{\perp} m_{D}$ for several values of $g$. We have described in detail how we evaluate $\mathcal{W}_{\mathcal{R}}^{(2)}\left(x_{\perp}\right)$ in sections III and IV. 

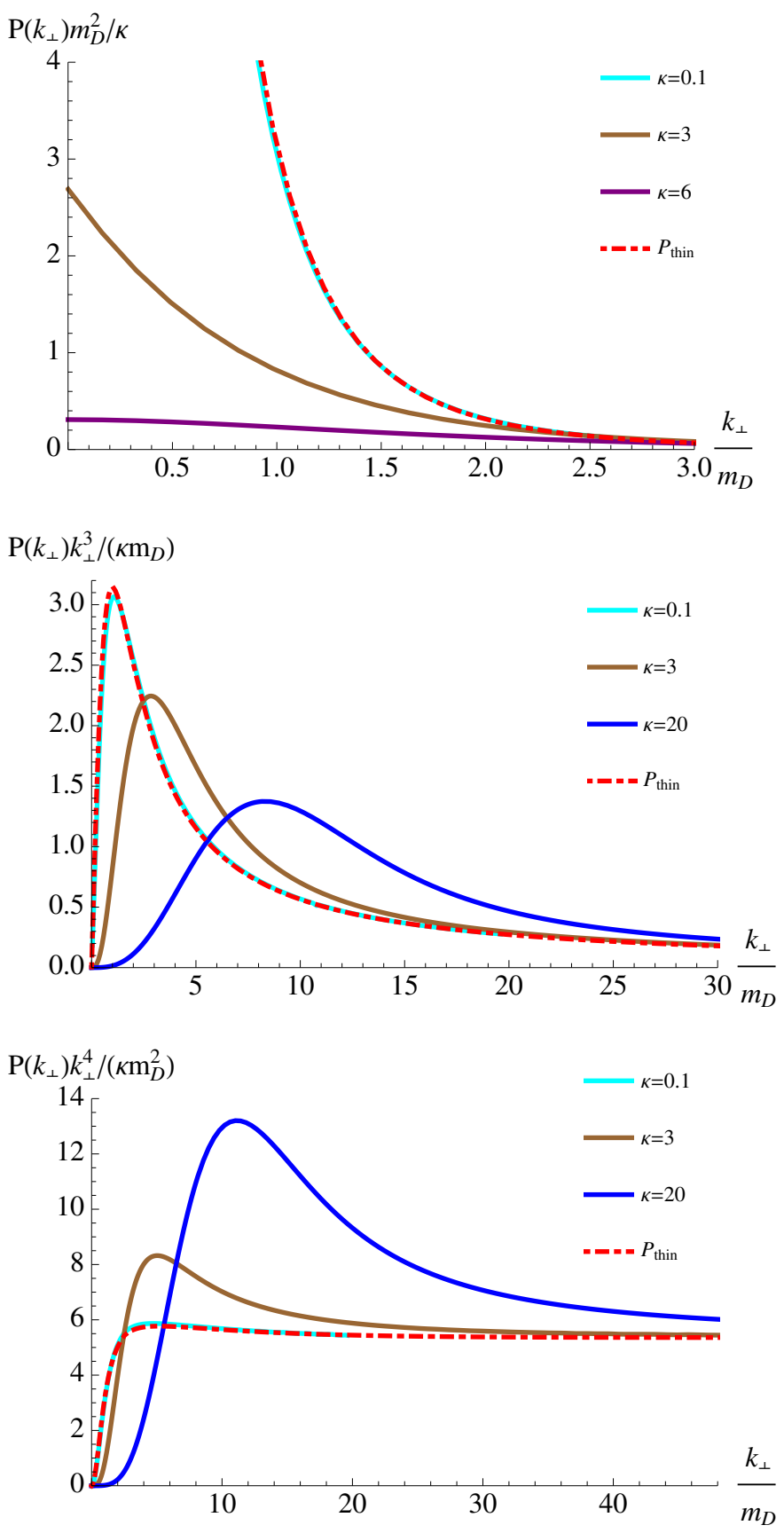

Figure 10. In the top panel, we plot our full result for the probability distribution $P\left(k_{\perp}\right)$, after resumming the contributions of $L$-enhanced diagrams. We show our results at three different values of $\kappa\left(\kappa\right.$ increases from top to bottom at low $\left.k_{\perp}\right)$, and confirm that at $\kappa=0.1$ our result agrees with $P_{\text {thin }}\left(k_{\perp}\right)$. In the middle and lower panels, we multiply $P\left(k_{\perp}\right)$ by $k_{\perp}^{3}$ and $k_{\perp}^{4}$ in order to highlight the behavior at intermediate and large $k_{\perp}$, as well as at a larger value of $\kappa$, namely $\kappa=20$. The gauge coupling constant is $g=0.1$ throughout. All the probability distributions are normalized as in (1.3). 


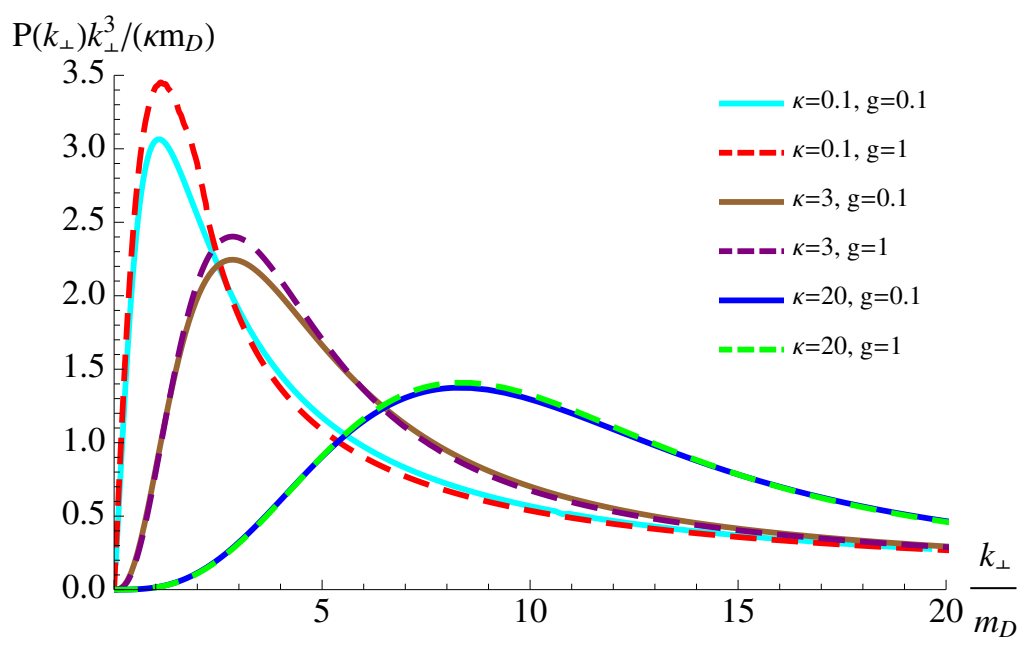

Figure 11. Probability distribution $P\left(k_{\perp}\right)$ at three different values of $\kappa$, multiplied by $k_{\perp}^{3}$ and plotted as in the middle panel of figure 10, but for two different values of $g$.

In the top panel of figure 10, we present our numerical results for the fully resummed probability distribution $P\left(k_{\perp}\right)$ that describes momentum broadening in a weakly coupled plasma with gauge coupling constant $g=0.1$. We show our results for three different values of the thickness of the medium $L$, meaning three different values of $\kappa$. We have also plotted $P_{\text {thin }}\left(k_{\perp}\right)$, and we see that when $\kappa=0.1$ the medium is so thin that $P\left(k_{\perp}\right) \simeq P_{\text {thin }}\left(k_{\perp}\right)$, meaning that there is no need to resum $L$-enhanced diagrams. Although $P_{\text {thin }}\left(k_{\perp}\right) \propto \kappa$, meaning that $P_{\text {thin }}\left(k_{\perp}\right) / \kappa$ in the figure 10 is $\kappa$-independent, our full result $P\left(k_{\perp}\right)$ has nontrivial $\kappa$-dependence at small $k_{\perp}$. This $\kappa$-dependence is better seen in the middle panel of the figure 10 , where we plot $k_{\perp}^{3} P\left(k_{\perp}\right)$. Note that the mean value of $k_{\perp}^{2}$, which is proportional to the jet quenching parameter $\hat{q}$ that we shall discuss in section V.C, is given by the area under the curves in this middle panel. We see from the figure that increasing $\kappa$ steadily shifts probability density away from small $k_{\perp}$, pushing it out to larger and larger $k_{\perp}$. This makes sense: as you make the medium thicker, the hard parton spends more time travelling through the medium, getting kicked, and so can pick up more and more transverse momentum. Finally, in the third panel of figure 10 we highlight the behavior of $P\left(k_{\perp}\right)$ at large $k_{\perp}$. We see that for any value of $\kappa$ at large enough $k_{\perp}$ the probability distribution $P\left(k_{\perp}\right)$ approaches $P_{\text {thin }}\left(k_{\perp}\right)$, meaning that resummation of $L$ enhanced diagrams is unnecessary. This is reasonable on physical grounds: for any value of $\kappa$ there will be some $k_{\perp}$ that is so large that the most probable way of picking up this improbably large $k_{\perp}$ is via a single scattering, which is described by $P_{\text {thin }}\left(k_{\perp}\right)$.

We expect from figure 9 , and confirm in figure 11, that once we plot $P\left(k_{\perp}\right)$ relative to $k_{\perp} / m_{D}$, there is little remaining $g$-dependence. We see from figure 11 that the $g$ dependence is at most about $10 \%$ at $\kappa=0.1$, and even much smaller than that at $\kappa=20$. Note that what figure 11 demonstrates is that, when plotted in this way, our results are insensitive to increasing $g$ at fixed $\kappa$. Increasing $g$ while holding $\kappa$ fix requires reducing $L$. 
If, instead, we increase $g$ at fixed $L$, this corresponds to increasing $\kappa$ - which (we see in figures 10 and 11 and below) has a marked effect on our results.

The $\kappa$-dependence of $P\left(k_{\perp}\right)$ at small $k_{\perp}$ manifest in figure 10 is interesting. For very small $\kappa, P\left(k_{\perp}\right) \simeq P_{\text {thin }}\left(k_{\perp}\right)$, which diverges proportional to $1 / k_{\perp}^{2}$ at small $k_{\perp}$, as in (5.5). Plotting our results at many more values of $\kappa$ than we have shown in figure 10 indicates that $P\left(k_{\perp}\right)$ diverges as $k_{\perp} \rightarrow 0$ if $\kappa<2$ and is finite at $k_{\perp}=0$ for $\kappa \geq 2$, and indicates that $P\left(k_{\perp}\right)$ is linear in $k_{\perp}$ at small $k_{\perp}$ if $\kappa=3$ (as illustrated in figure 10) and is quadratic in $k_{\perp}$ at small $k_{\perp}$ if $\kappa \geq 4$. All these features of the behavior of $P\left(k_{\perp}\right)$ in the $k_{\perp} \rightarrow 0$ limit can be demonstrated analytically, via approximating $P_{\text {thin }}\left(k_{\perp}\right)$ by $P_{\text {thin }}^{\mathrm{AGZ}}\left(k_{\perp}\right)$ as is valid for $k_{\perp} \rightarrow$ 0 , and then resumming $L$-enhanced diagrams. We present this analysis in appendix $\mathrm{F}$. We expect that the $L$-resummed $P^{\mathrm{AGZ}}\left(k_{\perp}\right)$ will agree with the full $P\left(k_{\perp}\right)$ at small $k_{\perp}$ because $P_{\text {thin }}^{\mathrm{AGZ}}\left(k_{\perp}\right)$ agrees with $P_{\text {thin }}\left(k_{\perp}\right)$ in this regime. The physical argument behind this expectation follows. For any value of $k_{\perp}$, resumming the $L$-enhanced diagrams means taking into account the possibility that the hard parton could pick up this $k_{\perp}$ via multiple scatterings, summing over the infinite number of ways of adding up individual kicks that yield $k_{\perp}$ in total. If we consider some $k_{\perp}$ that is small enough that $P_{\text {thin }}\left(k_{\perp}\right) \simeq P_{\text {thin }}^{\mathrm{AGZ}}\left(k_{\perp}\right)$, then getting this $k_{\perp}$ via multiple kicks that each transfer momenta much larger than $k_{\perp}$ is improbable. The $L$-resummation is therefore dominated by terms in which each of the multiple kicks transfers momenta that are comparable to or smaller than $k_{\perp}$, meaning that all the multiple kicks being resummed are small enough that AGZ is a good approximation. We therefore expect that, after resummation, $P\left(k_{\perp}\right) \simeq P^{\mathrm{AGZ}}\left(k_{\perp}\right)$. We confirm this by explicit calculation in appendix F. From the analysis in appendix $\mathrm{F}$ we then learn that as $k_{\perp} \rightarrow 0$, the probability distribution $P\left(k_{\perp}\right)$ includes a term proportional to $k_{\perp}^{2}$ as well as a (possibly nonanalytic) term proportional to $k_{\perp}^{\kappa-2}$, which dominates at small enough $k_{\perp}$ if $\kappa<4$. This term explains the qualitative features that we have described above.

We have seen that for $\kappa \geq 4$, the leading small- $k_{\perp}$ behavior of $P\left(k_{\perp}\right)$ is a constant minus a term quadratic in $k_{\perp}$. This immediately makes one think of a Gaussian. And, indeed, there is every reason to expect that at large $\kappa$ we should find a Gaussian probability distribution at small enough $k_{\perp}$ : large $\kappa$ means a thick medium, meaning that the hard parton picks up its transverse momentum via the sum of many kicks. This means that in the large- $\kappa$ regime we expect that momentum broadening can be thought of as diffusion in transverse momentum space, with a Gaussian probability distribution $P\left(k_{\perp}\right)$ arising via the central limit theorem. In figure 12 , we illustrate $P\left(k_{\perp}\right)$ for three large values of $\kappa$, showing that at small enough $k_{\perp}$ we do indeed find a Gaussian probability distribution. For each $\kappa$, we fit a Gaussian of the form

$$
P_{\text {fit }}\left(k_{\perp}\right)=A \exp \left(-a k_{\perp}^{2}\right)
$$

to $P\left(k_{\perp}\right)$ at low $k_{\perp}$. We determine $A$ by requiring that $P_{\text {fit }}(0)=P(0)$ and then determine the width parameter $a$ by fitting the quadratic dependence of $\log P\left(k_{\perp}\right)$ around $k_{\perp}=0$, i.e. by fitting the brown dashed parabolas to the results shown as the solid blue curves in the right panels of figure 12. (Although $P\left(k_{\perp}\right)$ is a normalized probability distribution, $P_{\text {fit }}$ is not normalized since as we can see in figure 12 it has less weight in its high- $k_{\perp}$ tail.) In order to gauge the range of $k_{\perp}$ out to which the probability distribution $P\left(k_{\perp}\right)$ is well 

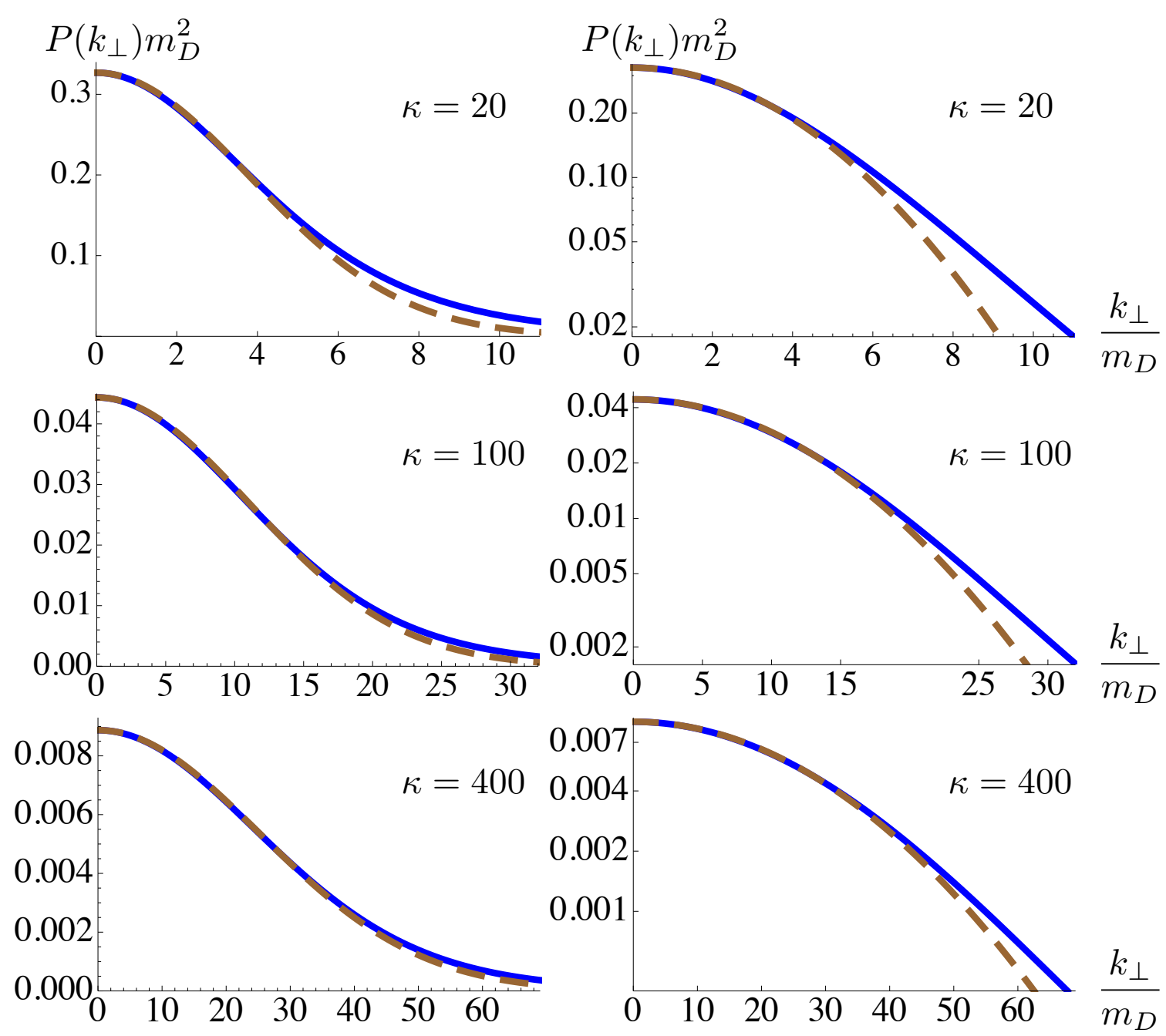

Figure 12. The solid blue curves show the probability distribution $P\left(k_{\perp}\right)$ describing momentum broadening in a weakly coupled plasma with $g=0.1$ at three different large values of $\kappa$ plotted on linear (left panels) and $\log$ (right panels) scales. For each $\kappa$, with the brown dashed curves we show a Gaussian fit of the form $P_{\text {fit }}\left(k_{\perp}\right)=A \exp \left(-a k_{\perp}^{2}\right)$ to $P\left(k_{\perp}\right)$ at low $k_{\perp}$. We find $a m_{D}^{2}=0.0345$, 0.00410 and 0.000792 for $\kappa=20,100$ and 400. We see from the figures that at larger $\kappa$ the Gaussian fit is a good approximation to $P\left(k_{\perp}\right)$ out to larger $k_{\perp} / m_{D}$, out to larger $k_{\perp} \sqrt{a}$, and down to lower probabilities. This is the central limit theorem in action. To quantify where the Gaussian approximation breaks down, we introduce $k_{\perp}^{G}$, defined as the value of $k_{\perp}$ where the Gaussian fit deviates from the full result by $2 \%$. We find $k_{\perp}^{G}=4.95 m_{D}=0.92 / \sqrt{a}, k_{\perp}^{G}=19.51 m_{D}=1.25 / \sqrt{a}$ and $k_{\perp}^{G}=$ $51.44 m_{D}=1.45 / \sqrt{a}$ for $\kappa=20,100$ and 400. For any $\kappa$ no matter how large, if $k_{\perp}$ is large enough — in particular if $k_{\perp} \gg k_{\perp}^{G}$ - a power-law tail with $P\left(k_{\perp}\right) \propto 1 / k_{\perp}^{4}$ rises above the Gaussian.

approximated by the Gaussian $P_{\text {fit }}\left(k_{\perp}\right)$, we define $k_{\perp}^{G}$ as the value of $k_{\perp}$ where the Gaussian fit function starts to deviate from the actual result by $2 \%: P\left(k_{\perp}^{G}\right)-P_{\text {fit }}\left(k_{\perp}^{G}\right)=0.02 P\left(k_{\perp}^{G}\right)$. We see from figure 12 that the larger $\kappa$ is, the larger is the $k_{\perp}^{G}$, both in units of $m_{D}$ and in units of $1 / \sqrt{a}$. More and more of the integrated probability is found in the regime in 
which the probability distribution is Gaussian at larger and larger $\kappa$. This is the central limit theorem in action.

We can confirm the reliability of the Gaussian fit that we have done by comparing our results for the Gaussian width parameter $a$ to the value of $a$ for the resummed AGZ distribution $P^{\mathrm{AGZ}}\left(k_{\perp}\right)$ that we obtain in appendix F. As we have already emphasized, the resummed AGZ distribution is an excellent approximation to the full probability distribution at small $k_{\perp}$, which is also where we find Gaussian behavior. Resumming the AGZ distribution should therefore describe the physics well in the region where we perform the fit, with the additional benefit of making it possible to do the calculation almost completely analytically. As described in detail in appendix F, we fit the Gaussian (5.8) to $P^{\mathrm{AGZ}}\left(k_{\perp}\right)$ and find an expression for the width parameter $a$ :

$$
a m_{D}^{2}=a^{\mathrm{AGZ}} m_{D}^{2} \equiv \frac{1}{4} \frac{\int_{0}^{\infty} d x x^{3-\kappa} \exp \left[-\kappa K_{0}(x)\right]}{\int_{0}^{\infty} d x x^{1-\kappa} \exp \left[-\kappa K_{0}(x)\right]},
$$

with $K_{0}$ the modified Bessel function of the second kind. The values of $a$ that we obtain from this expression with $\kappa$ set to 20, 100 and 400, namely $a^{\mathrm{AGZ}} m_{D}^{2}=0.0362,0.00410$ and 0.000763 , are all within a few percent of those that we have obtained by fitting to our full results in figure 12. Naive application of the central limit theorem would suggest that the width of the Gaussian, $\propto 1 / \sqrt{a}$, should increase like $\sqrt{L}$ and hence at fixed $g$ it should increase with increasing $\kappa$ like $\sqrt{\kappa}$. Instead we find both from our full results and from (5.9) that $1 / a$ grows slightly faster than linearly with $\kappa$, apparently including a $\kappa \log \kappa$ term as well as a term proportional to $\kappa$.

We have also investigated the $g$-dependence of $a$ by repeating the analysis shown in figure 12 at $g=1$, instead of $g=0.1$ as in the figure. In so doing we find that $a$ decreases by factors of 27000, 19000 and 16700 if we hold $L$ fixed, increasing $\kappa$ from the 20, 100 and 400 in the figure to 2000,10000 and 40000 . If instead we hold $\kappa$ fixed and increase $g$, we find that to a good approximation $1 / a$ increases like $g^{2}$, as in (5.9). Our results for the width parameter $a$ can therefore be summarized by writing $1 / a=m_{D}^{2} f(\kappa)$ for small values of $g$, with $f(\kappa)$ a function that grows slightly faster than linearly with $\kappa$ at large $\kappa$. This means that $1 / a$ grows slightly faster than like $g^{4}$ with increasing $g$ at fixed $L$.

To this point we have focussed on the Gaussian behavior illustrated in figure 12 but it is just as important to see in these plots that for any $\kappa$, no matter how large, at large enough $k_{\perp}$ you see the $P\left(k_{\perp}\right) \propto 1 / k_{\perp}^{4}$ behavior (5.7), which is the correct form for $P\left(k_{\perp}\right)$ in the asymptotic ultraviolet $k_{\perp} \rightarrow \infty$ limit. This apparent failure of the central limit theorem arises because the underlying probability distribution for a thin medium $P_{\text {thin }}\left(k_{\perp}\right)$ has a "fat tail": its power-law fall-off at large $k_{\perp}$ ensures that no matter how many scatterings are added up by resumming $L$-enhanced diagrams, i.e. no matter how thick the medium is, the behavior of $P\left(k_{\perp}\right)$ at large $k_{\perp}$ does not become Gaussian, it remains power-law. The physics behind this is that no matter how thick the medium there is a $k_{\perp}$ that is so large that the most probable way of picking up this much transverse momentum is via a single hard scattering. The "fat tail" of the $P_{\text {thin }}\left(k_{\perp}\right)$ distribution ensures that, for large enough $k_{\perp}$, although such single hard scatterings are rare they are more probable than picking up such a large $k_{\perp}$ from multiple scatterings. 
There are many previous analyses of momentum broadening via multiple scattering in a weakly coupled plasma in the literature in which approximations are made that result in a Gaussian form for $P\left(k_{\perp}\right)$. (See refs. [36, 51-53, 81, 82], for example, and see appendix D for further discussion.) It is important to realize that altehough at large $\kappa$ this is the correct form for "most of the probability", i.e. for a region in $k_{\perp}$ that contributes the lion's share of the normalization (1.3) of the probability density $P\left(k_{\perp}\right)$, at large enough $k_{\perp}$ the probability density is not Gaussian but rather a power law. And, in the $Q \rightarrow \infty$ limit (high jet-energy limit) in which the calculational framework within which we are working is controlled it is the large- $k_{\perp}$ power-law region of $P\left(k_{\perp}\right)$ that controls the jet quenching parameter $\hat{q} \propto\left\langle k_{\perp}^{2}\right\rangle$, as we shall discuss in the next subsection. We shall see there that there is nevertheless a sense in which $1 /(a L)$ can be thought of as a sort of "soft jet quenching parameter". More generally, it is interesting to ask how the width of the Gaussian component of $P\left(k_{\perp}\right)$ manifests itself in aspects of the phenomenology of jet quenching other than momentum broadening, even in the $Q \rightarrow \infty$ limit. However, $1 /(a L)$ is not the jet quenching parameter. And, in fact, we shall see that in the $Q \rightarrow \infty$ limit any dependence of $\hat{q}$ on $a$ is both subleading and implicit.

\subsection{Jet quenching parameter}

In this subsection we shall discuss the implications of our results for the jet quenching parameter $\hat{q}$, defined as

$$
\hat{q} \equiv \frac{\left\langle k_{\perp}^{2}\right\rangle}{L}=\frac{1}{L} \int \frac{d^{2} k_{\perp}}{(2 \pi)^{2}} k_{\perp}^{2} P\left(k_{\perp}\right) .
$$

Recall that in the large $k_{\perp}$ limit, the resummation discussed in section 2 becomes unnecessary, and we recover the limiting behavior of $P_{\text {thin }}\left(k_{\perp}\right)$ given in (5.7). We immediately see that the "fat tail" of the probability distribution, $P\left(k_{\perp}\right) \propto 1 / k_{\perp}^{4}$ at large $k_{\perp}$, makes $\hat{q}$ defined in (5.10) logarithmically divergent in the ultraviolet. Hence, the jet quenching parameter $\hat{q}$ is not well-defined in a weakly coupled plasma. This UV divergence has been noted by many authors before us, for example in refs. [22, 23, 66-69]. Nevertheless, the quantity $\hat{q}$ enters in many calculations of parton energy loss, even though its definition is based entirely upon momentum broadening, and we therefore want to compare our results for this quantity to those in the literature. To that end, we shall follow standard practice in much of the literature and regulate the integral in (5.10) with an ultraviolet cutoff $\Lambda_{U V}$, which is usually thought of as being a kinematic cutoff of order $\Lambda_{\mathrm{UV}} \sim(Q T)^{1 / 2}$, with $Q$ the energy of the hard parton. The thinking behind this conventional choice is that $\Lambda_{\mathrm{UV}}$ should be of order the maximum $k_{\perp}$ that the hard parton of energy $Q$ can pick up via a single scattering from a gluon in the medium with momentum of order $T$. More sophisticated, perhaps process-dependent, approaches are also possible $[66,68,83,84]$. In the $Q \rightarrow \infty$ limit in which the calculational framework within which we are working is controlled, $\Lambda_{\mathrm{UV}}$ is much greater than the $k_{\perp}^{G}$ below which $P\left(k_{\perp}\right)$ is Gaussian and it must be in the regime in which $P\left(k_{\perp}\right) \propto 1 / k_{\perp}^{4}$. We shall take this as given initially, but we shall later consider the possibility that $\Lambda_{\mathrm{UV}}$ may not always be so large for experimentally realizable values of $Q$.

Although our purpose in this subsection is to compare our results for $\hat{q}$ to those in the literature, we note here that for many purposes $\hat{q}$ may not be the most relevant 
parameter with which to characterize $P\left(k_{\perp}\right)$ in a weakly coupled plasma. Because $\hat{q}$ is ultraviolet-divergent in a weakly coupled plasma, in this setting it is determined by $P\left(k_{\perp}\right)$ at asymptotically large $k_{\perp}$, in a regime that contributes almost negligibly to the integrated probability. We have seen in figure 12 that although $P\left(k_{\perp}\right)$ has a "fat tail" that controls $\hat{q}$, most of the probability comes instead from the Gaussian region at lower $k_{\perp}$. So, in the case of a weakly coupled plasma the jet quenching parameter $\hat{q}$ describes only the tail, not the dog itself. In marked contrast, we shall see in the next subsection that in a plasma that is strongly coupled at all length scales, $P\left(k_{\perp}\right)$ is Gaussian at all momentum scales, and the jet quenching parameter $\hat{q}$ is finite, well-defined, and is the only parameter needed in order to characterize the entire probability distribution $P\left(k_{\perp}\right)[50,58]$.

We turn now to the calculation of $\hat{q}$, with its ultraviolet divergence regulated in the conventional way. In appendix $G$ we show that for a probability distribution of the form (2.15) the jet quenching parameter $\hat{q}$ takes the form

$$
\hat{q}=-\left.\frac{1}{L} \nabla^{2} \mathcal{W}_{\mathcal{R}}^{(2)}\right|_{x_{\perp}=0}
$$

where $\nabla^{2}$ is the Laplace operator in the transverse plane. Before proceeding to apply (5.11), we can make a very general point. If we were able to push our calculation through to all orders in perturbation theory, $\mathcal{W}_{\mathcal{R}}^{(2)}$ in $(2.15)$ would be replaced by the sum of connected diagrams in the exponent in $(2.14)$, which we can denote $\mathcal{W}_{\mathcal{R}}^{(c)}$. The calculation in appendix $G$ then still goes through, meaning that (5.11) becomes

$$
\hat{q}=-\left.\frac{1}{L} \nabla^{2} \mathcal{W}_{\mathcal{R}}^{(c)}\right|_{x_{\perp}=0}
$$

As we have discussed in section 2, the contribution of each connected diagram to $\mathcal{W}_{\mathcal{R}}^{(c)}$ is proportional to $L$. We then see that (5.12) constitutes a proof that $\hat{q}$ is independent of $L$ and, equivalently, of $\kappa$ to all orders in perturbation theory. So, $\hat{q}$ can only depend on $g$ and $T$ as well as on $\Lambda_{\mathrm{UV}}$.

We now apply (5.11) to our weak-coupling result (2.18) for $\mathcal{W}_{\mathcal{R}}^{(2)}$ and find

$$
\hat{q}=\frac{1}{L} \int \frac{d^{2} k_{\perp}}{(2 \pi)^{2}} k_{\perp}^{2} P_{\text {thin }}\left(k_{\perp}\right)
$$

which is divergent, as we expect. Even before we regulate the divergence, we notice the interesting result that $\hat{q}$ is the same as it would be if the probability distribution for momentum broadening were just $P_{\text {thin }}\left(k_{\perp}\right)$, instead of $P\left(k_{\perp}\right)$. We have seen in the previous subsection that the resummation of length-enhanced diagrams completely changes the shape of $P\left(k_{\perp}\right)$, but we now see that in a weakly coupled plasma this resummation has no effect on $\hat{q}$. This makes sense since in a weakly coupled plasma $\left\langle k_{\perp}^{2}\right\rangle$ is controlled by the ultraviolet power-law behavior of $P\left(k_{\perp}\right)$ which is unaffected by the resummation. In order to actually use the expression (5.13), we regulate it by introducing an ultraviolet cutoff $\Lambda_{\mathrm{UV}}$, as discussed above. (As long as $\Lambda_{\mathrm{UV}}$ is large enough, introducing the ultraviolet cutoff already in (5.10) would have been equivalent.) 
Because we are assuming $\Lambda_{\mathrm{UV}} \gg k_{\perp}^{G}$, we can present our results in a semi-analytical form. First we divide the integral over the magnitude of $k_{\perp}$ in the expression (5.13) for $\hat{q}$ into two parts, one from 0 to $k_{\perp}^{\prime}$ and the other from $k_{\perp}^{\prime}$ to $\Lambda_{\mathrm{UV}}$, with $k_{\perp}^{\prime}$ arbitrary except that it must satisfy $k_{\perp}^{\prime} \ll \Lambda_{\mathrm{UV}}, k_{\perp}^{\prime} \gg k_{\perp}^{G}$ and $k_{\perp}^{\prime} \gg T$. We denote the two contributions to $\hat{q}$ by

$$
\hat{q}=\hat{q}_{\mathrm{IR}}+\hat{q}_{\mathrm{UV}}
$$

Next, we observe that in the ultraviolet tail of $P_{\text {thin }}\left(k_{\perp}\right)$ the retarded propagator in eq. (3.4) is well approximated by the first order solution to the Dyson equation (3.1). This means that the Wightman propagator $D^{>}$, expressed in terms of the retarded propagator as in (2.19), takes the form

$$
D^{>}\left(q_{0}, k_{\perp}\right)=\left(1+f\left(q_{0}\right)\right) \frac{\operatorname{Im}\left(\Pi_{R}^{L}-\Pi_{R}^{T}\right)}{k_{\perp}^{2}\left(k_{\perp}^{2}+q_{0}^{2}\right)} .
$$

We then define the dimensionless coefficient

$$
b \equiv \int d q_{0} \frac{\left(1+f\left(q_{0}\right)\right)}{2 \pi^{2} T^{3}} \frac{k_{\perp}^{2}}{\left(k_{\perp}^{2}+q_{0}^{2}\right)} \frac{\operatorname{Im}\left(\Pi_{R}^{L}-\Pi_{R}^{T}\right)}{g^{2}} .
$$

Given the behavior of the imaginary part of the self-energy in the UV limit, the constant $b$ does not depend on either $k_{\perp}$ or $g$. We have defined $b$ so as to allow us to write a very compact expression for the probability distribution $P_{\text {thin }}\left(k_{\perp}\right)$ that is valid only in the ultraviolet limit,

$$
P_{\text {thin }}^{\mathrm{UV}}\left(k_{\perp}\right)=2 \pi b g^{4} C_{R} \frac{L T^{3}}{k_{\perp}^{4}},
$$

and from this we determine that the UV contribution to the jet quenching parameter is given by

$$
\hat{q}_{\mathrm{UV}}=b g^{4} C_{R} T^{3} \int_{k_{\perp}^{\prime}}^{\Lambda} \frac{d k_{\perp}}{k_{\perp}}=b g^{4} C_{R} T^{3} \log \frac{\Lambda}{k_{\perp}^{\prime}} .
$$

The arbitrary scale $k_{\perp}^{\prime}$ must be large enough that we can safely apply all the UV approximations just discussed. From its definition in eq. (5.16), we can calculate the value of $b$ explicitly. We find

$$
b=0.2035
$$

To check our results against those in the literature, we identify the factor corresponding to $b$ in the result (5.7) from ref. [66], finding

$$
b^{\mathrm{AD}}=\frac{1}{2 \pi} \frac{7 \zeta(3)}{4 \zeta(2)}=0.2035 .
$$

Our expression for $\hat{q}_{\mathrm{UV}}$ is therefore in excellent agreement with that obtained by Arnold and Dogan.

Turning now to the IR, we have

$$
\hat{q}_{\mathrm{IR}}=\frac{1}{2 \pi L} \int_{0}^{k_{\perp}^{\prime}} d k_{\perp} k_{\perp}^{3} P_{\text {thin }}\left(k_{\perp}\right) .
$$




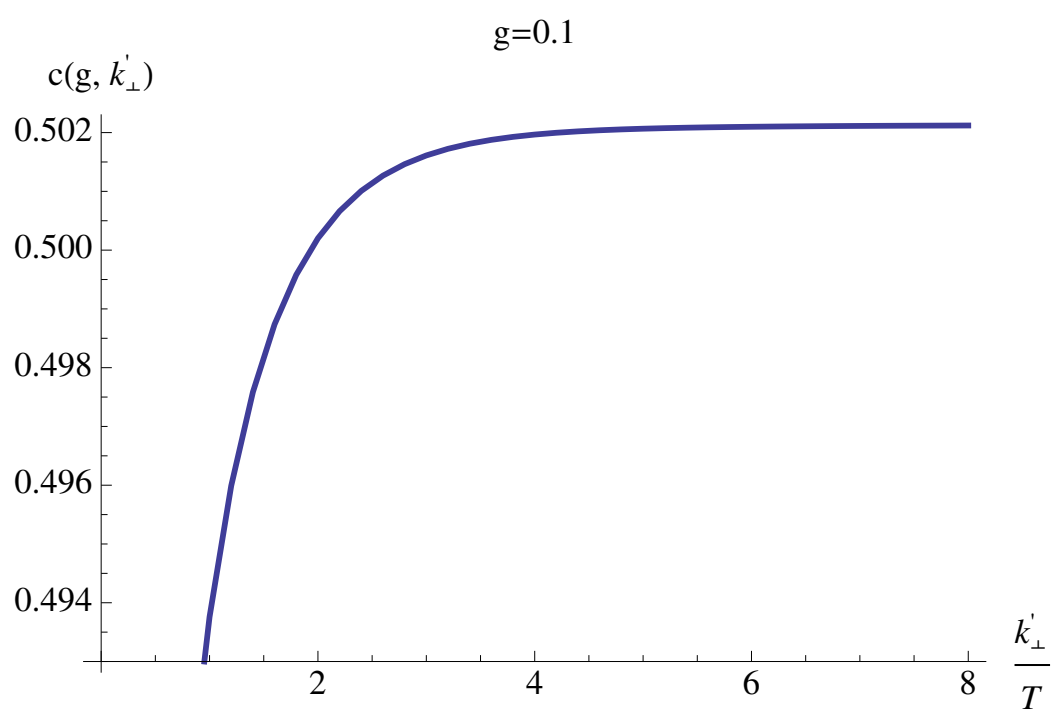

Figure 13. The function $c\left(g, k_{\perp}^{\prime}\right)$ as a function of $k_{\perp}^{\prime}$, with the gauge coupling set to $g=0.1$. For large enough $k_{\perp}^{\prime}, c\left(g, k_{\perp}^{\prime}\right)$ becomes constant.

Since we chose $k_{\perp}^{\prime}$ to be well into the UV region, the integral in (5.21) must depend logarithmically on $k_{\perp}^{\prime}$. To isolate this known logarithmic behavior, we define the function $c\left(g, k_{\perp}^{\prime}\right)$ via the expression

$$
\hat{q}_{\mathrm{IR}}=g^{4} C_{R} T^{3}\left[c\left(g, k_{\perp}^{\prime}\right)+b \log \frac{k_{\perp}^{\prime}}{T}\right]
$$

in which $\hat{q}_{\mathrm{IR}}$ is to be evaluated using (5.21) and which therefore serves to define $c$. The function $c\left(g, k_{\perp}^{\prime}\right)$ is plotted in figure 13 as a function of $k_{\perp}^{\prime}$ for $g=0.1$. We see that for large enough $k_{\perp}^{\prime}$, the function $c\left(g, k_{\perp}^{\prime}\right)$ becomes independent of $k_{\perp}^{\prime}$. And, we anyway needed to choose $k_{\perp}^{\prime} \gg T$ in order to control the behavior of $\hat{q}_{\mathrm{UV}}$. Upon making this choice, $c\left(g, k_{\perp}^{\prime}\right) \simeq c(g)$. The only way that properties of the probability distribution $P_{\text {thin }}\left(k_{\perp}\right)$ in the IR, and indeed in any region of $k_{\perp}$ except the UV, enter into the calculation of $\hat{q}$ is through the function $c(g)$. In some implicit way, $c(g)$ is related to the properties of the $P\left(k_{\perp}\right)$ distribution that we discussed in section 5.2, like for example the width $1 / \sqrt{a}$ of its Gaussian component at large $\kappa$. Notice, however, that $c(g)$ appears only in a subleading contribution to $\hat{q}$; the dominant contribution comes from the logarithmic UV divergence. We have computed $c(g)$ for several different $g$ values, obtaining

$$
c(g)=\left\{\begin{aligned}
1.0363 \text { for } g & =0.01 \\
0.5023 \text { for } g & =0.1 \\
0.0368 \text { for } g & =1 \\
-0.0795 \text { for } g & =2
\end{aligned}\right. \text {. }
$$

Finally, we combine the two contributions $\hat{q}_{\mathrm{UV}}$ in eq. (5.18) and $\hat{q}_{\mathrm{IR}}$ in eq. (5.22). 
Summing them, we find

$$
\hat{q}=g^{4} C_{R} T^{3}\left(b \log \frac{\Lambda_{\mathrm{UV}}}{T}+c(g)\right)
$$

where the dependence on the arbitrarily chosen $k_{\perp}^{\prime}$ has dropped out, as it must. Thus, at the end of the day we find that the jet quenching parameter is specified by the constant $b$ and the function of the coupling constant $c(g)$, as well as $T, \Lambda_{\mathrm{UV}}$ and $g$ itself. Our results for $b$ and $c(g)$ are given in (5.19) and (5.23), respectively. As a comparison, the AGZ distribution in (5.5) gives

$$
\hat{q}^{\mathrm{AGZ}}=g^{4} C_{R} T^{3} \frac{3}{4 \pi} \log \frac{\Lambda_{\mathrm{UV}}}{m_{D}},
$$

from which we can read off the coefficients

$$
b^{\mathrm{AGZ}}=\frac{3}{4 \pi}, \quad c^{\mathrm{AGZ}}(g)=-\frac{3}{4 \pi} \log \left(g \sqrt{\frac{3}{2}}\right) .
$$

The coefficients $b$ and $b^{\mathrm{AGZ}}$ differ by $\approx 15 \%$. This is the discrepancy between the ultraviolet tails of $P_{\text {thin }}\left(k_{\perp}\right)$ and $P_{\text {thin }}^{\mathrm{AGZ}}\left(k_{\perp}\right)$ (recall that $P_{\text {thin }}^{\mathrm{AGZ}}\left(k_{\perp}\right)$ is not valid in the ultraviolet) that we have already seen in figure 8 . The values of $c(g)$ and $c^{\mathrm{AGZ}}(g)$ are in good agreement for small values of $g$, and differ more for larger values of $g$, where $P_{\text {thin }}\left(k_{\perp}\right)$ starts to differ from $P_{\text {thin }}^{\mathrm{AGZ}}\left(k_{\perp}\right)$.

We can even plug in explicit values for the parameters in a range that is reasonable for jets produced in heavy ion collisions at the LHC. We pick the benchmark values $T=$ $300 \mathrm{MeV}$ and $L=5 \mathrm{fm}$ for the plasma temperature and thickness, respectively. We choose the UV cutoff to be $\Lambda_{\mathrm{UV}}=17 \mathrm{GeV}$. (This can be thought of as the kinematic limit for a $300 \mathrm{GeV}$ parton scattering off a $1 \mathrm{GeV}$ parton from the medium.) We consider the momentum broadening of a hard gluon, meaning that we set $C_{\mathcal{R}}=3$, and find

$$
\hat{q}=0.411 g^{4}(0.822+c(g)) \mathrm{GeV}^{2} \mathrm{fm}^{-1} .
$$

If we then choose $g=2$, corresponding to $\alpha_{\mathrm{QCD}}=0.32$, this would correspond to a jet quenching parameter $\hat{q} \simeq 4.9 \mathrm{GeV}^{2} \mathrm{fm}^{-1}$. With these choices of parameters, $\kappa \simeq 14.5$, large enough that the resummation of length-enhanced diagrams is certainly necessary in order to obtain $P\left(k_{\perp}\right)$. With $\kappa \simeq 14.5$, extrapolating from figure 12 we estimate $k_{\perp}^{G} \sim 4 m_{D}$, meaning that with the parameters we have chosen it is around $3 \mathrm{GeV}$. So, our assumption that $\Lambda_{\mathrm{UV}} \gg k_{\perp}^{G}$ is reasonable, as is our calculation in which $\hat{q}$ is dominated by the tail of the probability distribution $P\left(k_{\perp}\right)$.

Although the calculation breaks down if we do so, it is also interesting to speculate as to how its results differ if we attempt to use parameters more appropriate for the momentum broadening of hard partons produced in heavy ion collisions at RHIC. In the RHIC context, $\Lambda_{\mathrm{UV}}$ must be much smaller, first because the hard partons only have $Q \sim 30-40 \mathrm{GeV}$ and second because the temperature is somewhat lower. Likely, $\Lambda_{\mathrm{UV}}$ cannot be much more than $5 \mathrm{GeV}$. Also, the relevant values of $g$ must be somewhat larger at RHIC than at the LHC. With $\kappa$ increasing like $g^{2}$, according to figure 12 this will result 
in a larger $k_{\perp}^{G}$. This means that the assumption that $\Lambda_{\mathrm{UV}} \gg k_{\perp}^{G}$ has broken down, as has our calculation of (5.27). Of course, the whole calculational framework is breaking down too, for two independent reasons: $g$ is becoming uncomfortably large and we can no longer trust the $Q \rightarrow \infty$ limit as a guide.

Motivated both by jet quenching at RHIC and by the analysis of the strongly coupled plasma that we shall discuss in the next subsection, it is worth asking what happens if $\Lambda_{\mathrm{UV}}<k_{\perp}^{G}$ even though as we have just described our weakly coupled calculation is not at all under control in this regime. If we nevertheless apply our results, when $\Lambda_{\mathrm{UV}}$ lies within the regime in which $P\left(k_{\perp}\right)$ is well fit by a Gaussian of the form (5.8) then the power-law tail of the probability distribution is irrelevant in the calculation of the jet quenching parameter, since the integral in (5.13) is cutoff before the power-law tail makes its appearance. $\hat{q}$ is determined entirely from the Gaussian region, and is given in terms of the width $1 / \sqrt{a}$ of the Gaussian (5.8) by

$$
\hat{q}_{\mathrm{soft}}=\frac{1}{a L},
$$

where we have introduced the subscript "soft" to remind ourselves that this result is only valid if $\Lambda_{\mathrm{UV}}<k_{\perp}^{G}$ and in particular is not valid in the $Q \rightarrow \infty$ limit where the full calculation is controlled. Even though $\hat{q}_{\text {soft }}$ is not the actual jet quenching parameter $\hat{q}$ (which is defined in the $Q \rightarrow \infty$ limit and is given by (5.24)) since $\hat{q}_{\text {soft }}$ is determined by the width of the Gaussian that describes the lion's share of the probability in $P\left(k_{\perp}\right)$ rather than by the power-law tail it could certainly turn out that $\hat{q}_{\text {soft }}$ is more relevant to the phenomenology of jet quenching than $\hat{q}$ itself. Our attempt to plug numbers into our results suggests that this circumstance is more likely to arise for jets produced in heavy ion collisions at RHIC and is less likely to arise in the case of the highest energy jets produced in heavy ion collisions at the LHC.

\subsection{From weak to strong coupling}

We have calculated the probability distribution $P\left(k_{\perp}\right)$ for momentum broadening in a weakly coupled quark-gluon plasma in QCD. We anticipate that our calculation is only quantitatively reliable for $g<1$. For example, we argued in section 5.2 based upon evidence visible in figure 8 that the matching that we describe in section IV is not quantitatively reliable at $g=1$ and $g=2$, although it is gratifying that the small kinks in the $g=1$ and $g=2$ curves in figure 8 have no visible effects in figure 9 , and therefore no visible effects on our results, as plotted in figures 10,11 and 12. Although it is important on theoretical grounds that we have control of the calculation for $g<1$, this is of little phenomenological interest. The smallest values of $g$ that are typically used in comparisons to data from heavy ion collisions at RHIC and the LHC are around $g \sim 2$. (Note that $g=2$ corresponds to $\alpha_{\mathrm{QCD}} \simeq 0.32$, in many other contexts a weak coupling.) There are other good reasons beyond the large value of $g$ not to trust a weakly coupled description of the plasma produced in heavy ion collisions at RHIC and the LHC, chief among them being the suite of evidence that this plasma is a strongly coupled liquid with a shear viscosity that is so small that no description in terms of weakly interacting quark and gluon quasiparticles 
can be self-consistent. That said, it is nevertheless natural to ask, at least at a qualitative level, what our results suggest for $P\left(k_{\perp}\right)$ at $g \sim 2$ and beyond. At a qualitative level, the answer is provided by figure 12. By far the most important consequence of increasing $g$ at fixed $L$ is the increase in $\kappa$, and we see from figure 12 that increasing $\kappa$ makes the probability distribution $P\left(k_{\perp}\right)$ for momentum broadening "more and more Gaussian." That is, $P\left(k_{\perp}\right)$ is well-approximated as Gaussian out to larger and larger $k_{\perp}$ and for a larger and larger fraction of the total probability, pushing the appearance of the power-law behavior that must be present at asymptotic $k_{\perp}$ out to larger and larger $k_{\perp}$.

The qualitative expectations for the behavior of $P\left(k_{\perp}\right)$ at strong coupling that we have gleaned by looking at how our results behave at large $\kappa$ are nicely borne out in the strongly coupled plasma of $\mathcal{N}=4$ supersymmetric Yang-Mills (SYM) theory. Taking advantage of the fact that this theory, at both zero and nonzero temperature, has a dual gravitational description [85-92], holographic calculations of many aspects of its plasma phase have been used to gain varied insights into the physics of strongly coupled plasma more generally (for a review, see ref. [93]). Of interest to us, the expectation value of the Wilson loop in eq. (2.3), and from it the probability distribution for momentum broadening, have been calculated for $\mathcal{N}=4 \mathrm{SYM}$ theory in the strong coupling and large- $N_{c}$ limit $[50,58]$. For a propagating gluon in the adjoint representation, the result reads

$$
P^{\mathrm{SYM}}\left(k_{\perp}\right)=\frac{4 \tilde{a}}{\pi \sqrt{\lambda} T^{3} L} \exp \left[-\frac{\tilde{a} k_{\perp}^{2}}{\pi^{2} \sqrt{\lambda} T^{3} L}\right]
$$

where $\lambda \equiv g^{2} N_{c}$ is the 't Hooft coupling, assumed large, and where $\tilde{a} \equiv$ $\sqrt{\pi} \Gamma(5 / 4) / \Gamma(3 / 4) \approx 1.311$. We see immediately that in this theory, whose plasma is strongly coupled at all scales, $P\left(k_{\perp}\right)$ is Gaussian out to arbitrarily large $k_{\perp}$, which is consistent at a qualitative level with the expectations derived from extending our calculation for a weakly coupled plasma to larger values of $g$ and hence $\kappa$, as we have described above. At a qualitative level, the lesson from (5.29) is that in a strongly coupled plasma momentum broadening should be thought of as diffusion in transverse momentum space, even though in the calculation behind (5.29) there is no thin-medium regime, no analogue of starting with some $P_{\text {thin }}$ and resumming length-enhanced diagrams, and hence no picture of multiple scattering off quasiparticles building up a Gaussian $P\left(k_{\perp}\right)$ via the central limit theorem. In a strongly coupled plasma, $P\left(k_{\perp}\right)$ is always Gaussian at any $k_{\perp}$, for any $L$. At a qualitative level, such a regime can be approached starting from a weakly coupled plasma either by increasing the coupling or by increasing $L$, either of which corresponds to increasing $\kappa$.

Although the qualitative picture that we have just sketched is pleasing, it is important to note that at a quantitative level the Gaussian probability distribution (5.29) is quite different from the Gaussian that we obtained by fitting to our results for a weakly coupled plasma at large $\kappa$ in figure 12. In particular, the width of the Gaussian in (5.29) increases with increasing $g$ only like $\lambda^{1 / 4} \sim \sqrt{g}$ while we found in section 5.2 that the width $1 / \sqrt{a}$ of the Gaussians in figure 12 increase with increasing $g$ somewhat faster than $g^{2}$.

The result quoted in eq. (5.29) was derived in the $\lambda \rightarrow \infty$ limit. For large but finite 't Hooft coupling $\lambda$, we cannot conclude that $P\left(k_{\perp}\right)$ will be Gaussian at all scales. It is reasonable to expect that in this case like at weak coupling $P\left(k_{\perp}\right)$ will be Gaussian only 
for $k_{\perp}$ less than some $k_{\perp}^{G}$, with $k_{\perp}^{G} \rightarrow \infty$ as $\lambda \rightarrow \infty$ and consequently $\kappa \rightarrow \infty$. The form of $P\left(k_{\perp}\right)$ for $k_{\perp}>k_{\perp}^{G}$, where the Gaussian description breaks down, is not known. However, since there are no quasiparticles off which hard scattering can occur in the $\mathcal{N}=4 \mathrm{SYM}$ theory plasma with large but finite $\lambda$, we anticipate that even where $P\left(k_{\perp}\right)$ is not Gaussian it will continue to fall off more rapidly than any power of $k_{\perp}$ at large $k_{\perp}$.

We illustrate both the qualitative and the quantitative comparisons that we have just made between $P\left(k_{\perp}\right)$ in weakly coupled QCD plasma and $P^{\mathrm{SYM}}\left(k_{\perp}\right)$ in strongly coupled $\mathcal{N}=4$ SYM plasma in figure 14 by plotting both for $g=1$ and $g=2$. In QCD, $g=1$ and 2 correspond to $\alpha_{\mathrm{QCD}}=0.08$ and 0.32 while in $\mathcal{N}=4$ SYM theory with $N_{c}=3$, these couplings correspond to $\lambda=3$ and 12. It is interesting to note that $g=2$ is in a regime in which $\alpha_{\mathrm{QCD}}=0.32$ is considered a weak coupling in some contexts and $\lambda=12$ is considered a strong coupling in some contexts. We perform the comparison for a plasma with temperature $T=300 \mathrm{MeV}$ that is $L=5 \mathrm{fm}$ thick. With these choices, $g=1$ and 2 correspond to $\kappa=3.6$ and $\kappa=14.5$. We see in figure 14 that upon increasing $g$ from 1 to 2 , the width of $P\left(k_{\perp}\right)$ for the weakly coupled QCD plasma increases much more rapidly than the width of the Gaussian (5.29) for the strongly coupled plasma does, as we have described above. Perhaps the most striking aspect of figure 14 is just how similar $P\left(k_{\perp}\right)$ with $g=2$ and $P^{\mathrm{SYM}}\left(k_{\perp}\right)$ with $g=2$ and hence $\lambda=12$ are, in particular when plotted as in the top and middle panels. This is an indication that at this value of the coupling the strongly coupled and weakly coupled perspectives yield comparable descriptions of momentum broadening for low and moderate values of $k_{\perp} / T$. We see in the bottom panel of figure 14, however, that the physics of the two descriptions is completely different at large- $k_{\perp}$, where we see that the probability distribution $P\left(k_{\perp}\right)$ has a power-law tail only for the weakly coupled plasma.

We further illustrate the sharp distinction between the behavior of $P\left(k_{\perp}\right)$ at large $k_{\perp}$ in weakly and strongly coupled plasma in figure 15 . Because the probability distribution for the plasma that is strongly coupled at all scales is Gaussian whereas that for the weakly coupled plasma is proportional to $1 / k_{\perp}^{4}$ at large $k_{\perp}$, no matter how large the coupling is there is always a $k_{\perp}$ beyond which $P\left(k_{\perp}\right)$ is greater in the weakly coupled plasma than in the strongly coupled plasma. This behavior, which at first hearing may sound counterintuitive, reflects the presence of point-like quasiparticles in the weakly coupled plasma. This means that, as Rutherford could have understood, although the probability for large-angle, large- $k_{\perp}$, scattering is always low it is much larger in a plasma containing point-like scatterers than it would be in a liquid plasma with no quasiparticles at any length-scale like the strongly coupled plasma of $\mathcal{N}=4$ SYM theory. In figure 15 we plot the integrated probability that a hard parton propagating through $L=5 \mathrm{fm}$ of either the weakly coupled QCD plasma or the strongly coupled $\mathcal{N}=4 \mathrm{SYM}$ plasma with temperature $T=300 \mathrm{MeV}$ picks up a transverse momentum kick $k_{\perp}>k_{\perp}^{\mathrm{min}}$. As we can see, in the strongly coupled plasma with its Gaussian $P^{\mathrm{SYM}}\left(k_{\perp}\right)$, this integrated probability is completely negligible for $k_{\perp}^{\min } \gtrsim 40 T$. In stark contrast, if we assume a weakly coupled QCD plasma and then set $g=2$, this integrated probability is still more than half a percent for $k_{\perp}^{\min }=80 T$. So, although the two probability distributions are quite similar in the regime of $k_{\perp}$ which is probable - indicating that momentum broadening for most partons would be comparable in these two cases - rare hard, large-angle, scatterings will be very much more common if the weakly coupled QCD analysis yields a reasonable approximation. 

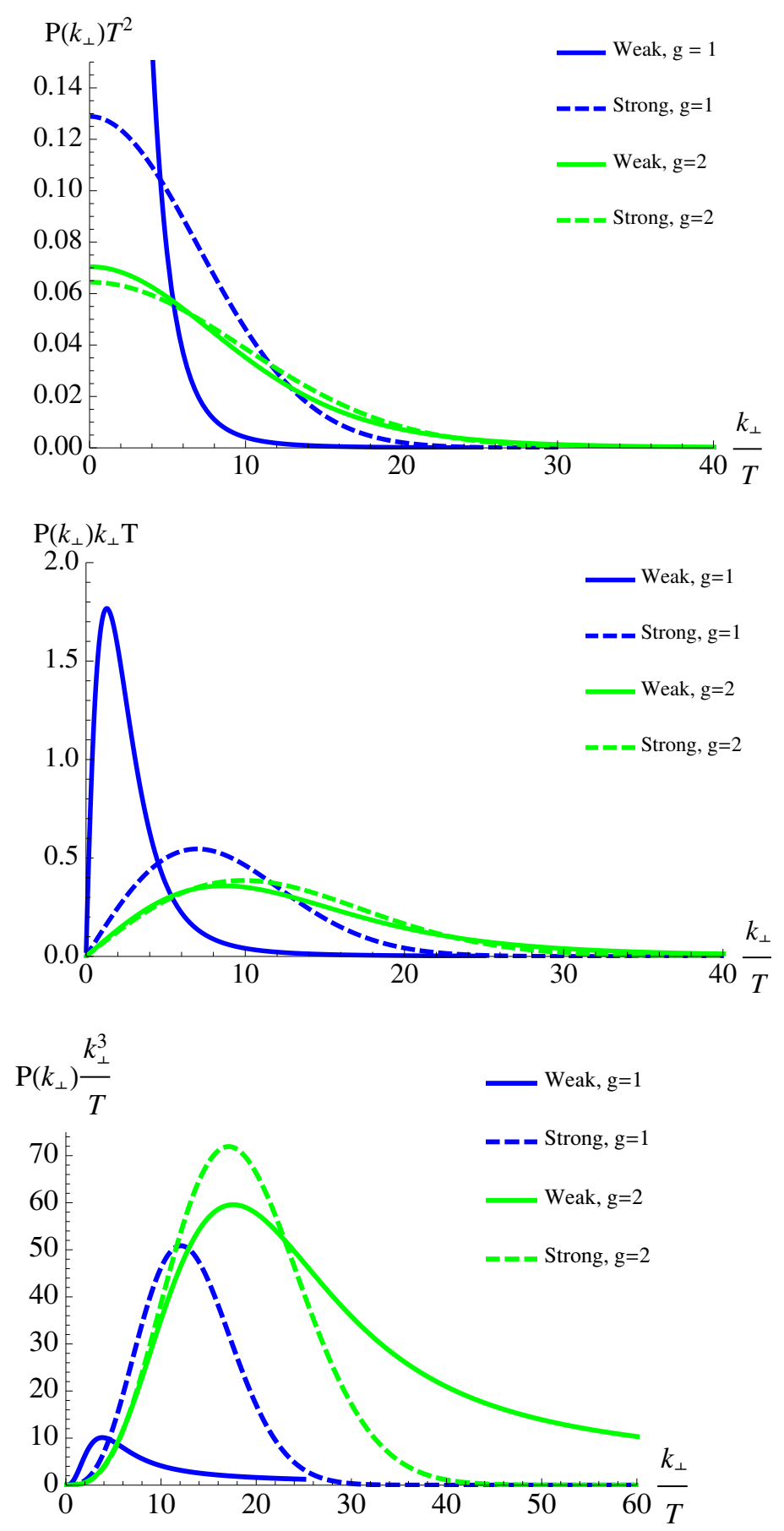

Figure 14. Probability distributions for momentum broadening $P\left(k_{\perp}\right)$ for weakly coupled QCD plasma with $g=1$ (continuous blue curve) and $g=2$ (continuous green) and $P^{\mathrm{SYM}}\left(k_{\perp}\right)$ for strongly coupled $\mathcal{N}=4 \mathrm{SYM}$ plasma with $N_{c}=3$ and $g=1$ (blue dashed) and $g=2$ (green dashed). The curves are for propagation of a hard gluon through a region of plasma with thickness $L=5 \mathrm{fm}$, and temperature $T=300 \mathrm{MeV}$. 

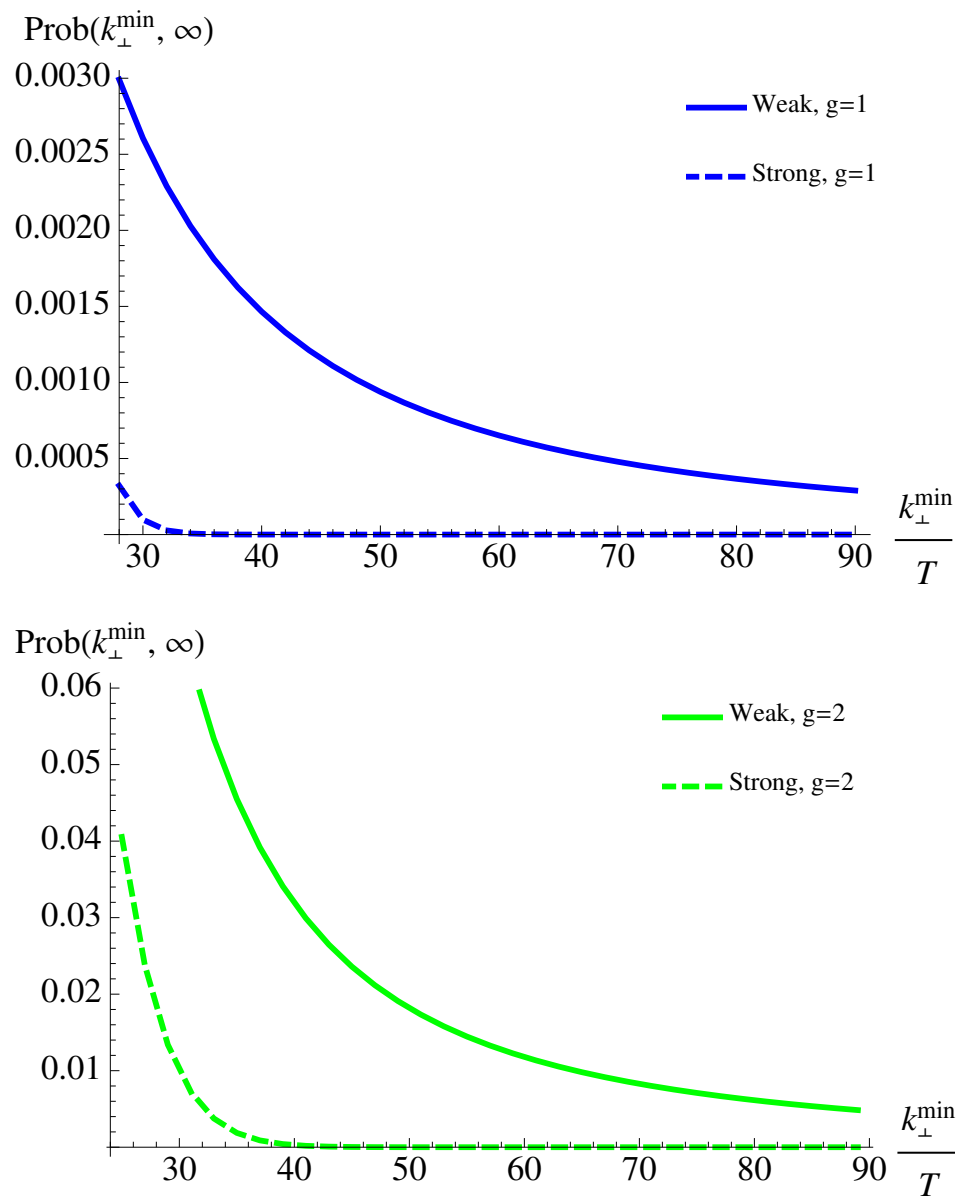

Figure 15. Probability that a hard gluon receives a transverse momentum greater than $k_{\perp}^{\min }$ after propagating a distance $L$ through a weakly coupled QCD plasma or a strongly coupled $\mathcal{N}=4$ SYM plasma with temperature $T$ and coupling constant $g$. Values of $g, T$ and $L$ as well as color conventions for the curves are all as in figure 14.

If we evaluate our results for the momentum broadening of a hard quark rather than a hard gluon, the conclusions of the above paragraph become even stronger. The widths of the Gaussian probability distributions $P^{\mathrm{SYM}}\left(k_{\perp}\right)$ describing the momentum broadening of a hard quark in the strongly coupled $\mathcal{N}=4$ SYM plasma are half as wide as those in figure 14 [58], meaning that the dashed curves plotted in figure 15 are pushed down so much that they are indistinguishable from the horizontal axis across the whole range of $k_{\perp}$ in figure 15. At weak coupling, in the ultraviolet $P\left(k_{\perp}\right)$ is proportional to $C_{\mathcal{R}}$, meaning that the solid curves in figure 15 get multiplied by a factor of $4 / 9$ if one treats a hard quark instead of a hard gluon. So, in a weakly coupled QCD plasma with $g=2$ and $L$ and $T$ as in figure 15 the integrated probability that a hard quark picks up $k_{\perp}^{\min }=30 T(60 T)$ or more in transverse momentum is more than two percent (about half a percent), while either of those integrated probabilities is completely negligible in the strongly coupled plasma with its Gaussian $P^{\mathrm{SYM}}\left(k_{\perp}\right)$. Because QCD is asymptotically free, its strongly coupled 
liquid quark-gluon plasma must emerge from weakly coupled quarks and gluons that can be resolved at short enough length scales. We therefore expect that for large enough $k_{\perp}$ our weakly coupled QCD analysis yields a reasonable approximation to $P\left(k_{\perp}\right)$, meaning that we expect that although large-angle scattering is rare it will be very much more common than it would be if the quark-gluon plasma were a strongly coupled liquid at all length scales.

\section{Outlook}

We have calculated the probability distribution $P\left(k_{\perp}\right)$ for an energetic parton that propagates for a distance $L$ without radiating through weakly coupled quark-gluon plasma with temperature $T$ to pick up transverse momentum $k_{\perp}$. Our calculation is built upon Soft Collinear Effective Theory (SCET), but to date we have not used much of the power of SCET, which could in future be brought to bear on the question of calculating corrections (for example in the ratio of $T$ to the parton energy) to leading order results like ours. Before doing this, however, the most pressing next steps beyond our calculation are to include the radiation of collinear and soft gluons.

To date, SCET has been used to relate $P\left(k_{\perp}\right)$ in any medium to $\mathcal{W}_{\mathcal{R}}\left(x_{\perp}\right)$, the expectation value in that medium of a Wilson loop with two long light-like sides separated in the transverse direction by a distance $x_{\perp}$. What we have done here is to use standard methods from real time thermal field theory, including Hard Thermal Loop resummation where needed (see section IV for an explanation of where it is needed), to calculate the expectation value $\mathcal{W}_{\mathcal{R}}\left(x_{\perp}\right)$ and from it the probability for momentum broadening $P\left(k_{\perp}\right)$, for the case in which the medium is weakly coupled quark-gluon plasma in thermal equilibrium at temperature $T$, as in $\mathrm{QCD}$ at temperatures that are sufficiently high that the QCD coupling constant $g$ is less than one.

We first obtained $P_{\text {thin }}\left(k_{\perp}\right)$ for a "thin medium", in which $\kappa$ (proportional to $g^{2} L T$ and defined in (5.1)) is much less than one. We have checked that our results in this regime agree with previous determinations, both at large and small $k_{\perp}$. Although it is a stretch to apply a calculation that requires $g<1$ to plasma at temperatures such that $g \sim 2$ we do so anyway, since the lowest estimates of the coupling constant in the plasma produced in heavy ion collisions at RHIC and the LHC are in this range. Doing this requires consideration of values of $\kappa$ that are significantly greater than one. (A reasonable choice like $L \sim 5 \mathrm{fm}$ and $T \sim 300 \mathrm{MeV}$ corresponds to $\kappa \sim 14$ if $g \sim 2$.) Handling $\kappa>1$ required us to resum an infinite class of length-enhanced planar diagrams in order to obtain the leading behavior of $P\left(k_{\perp}\right)$. After resumming the $L$-enhanced diagrams we obtain results valid for any value of $\kappa$ including $\kappa \gg 1$. We find that in a weakly coupled plasma the properties of the plasma enter the calculation of $P\left(k_{\perp}\right)$ only through the retarded gluon propagator. We computed the self-energy therein using real time thermal field theory, including Hard Thermal Loop resummation in the infrared. We find that the form of $P\left(k_{\perp}\right)$ changes qualitatively between $\kappa<2$, where it diverges at small $k_{\perp}$, and $\kappa>4$ where it looks Gaussian at small $k_{\perp}$. Our entire discussion is only valid to leading order in the coupling constant $g$. It will be interesting in the future to quantify the effects of length-enhancement at large $\kappa$ for existing next-to-leading order calculations [68]. 
At large $\kappa$, we find that $P\left(k_{\perp}\right)$ takes the form of a Gaussian at small $k_{\perp}$ with a power-law tail, proportional to $1 / k_{\perp}^{4}$, at large $k_{\perp}$. As $\kappa$ increases, more and more of the integrated probability resides in the Gaussian, with the power-law tail contributing less and less. Since $\kappa \rightarrow \infty$ can equally well be thought of as the strong coupling limit as the large- $L$ limit, at a qualitative level our large- $\kappa$ results are pleasingly consistent with the known behavior of $P\left(k_{\perp}\right)$ in the plasma of large- $N_{c} \mathcal{N}=4$ SYM theory in the strong coupling limit. In this theory, $P\left(k_{\perp}\right)$ is precisely Gaussian, with no power-law tail at all. Although the widths of the Gaussian in the strongly coupled $\mathcal{N}=4$ SYM plasma and of the Gaussian that characterizes $P\left(k_{\perp}\right)$ in the weakly coupled QCD plasma for all but improbably large values of $k_{\perp}$ do not agree quantitatively, and in particular have different $g$-dependence, it is interesting to note that they are remarkably similar for $g=2$, a value of the coupling that can reasonably be thought of as weak since $g^{2} /(4 \pi)$ is small and that can reasonably be thought of as strong since $g^{2} N_{c}$ is large for $N_{c}=3$.

The most noteworthy difference between momentum broadening in the weakly coupled QCD plasma and in the strongly coupled $\mathcal{N}=4 \mathrm{SYM}$ plasma is at large $k_{\perp}$. Because it has $P\left(k_{\perp}\right) \propto 1 / k_{\perp}^{4}$ at large $k_{\perp}$, no matter how small $\kappa$ or $g$ is there will always be a $k_{\perp}$ for which the weakly coupled plasma has a larger $P\left(k_{\perp}\right)$ than that in any strongly coupled plasma. In a weakly coupled plasma, the physics of momentum broadening is different at small $k_{\perp}$ than at large $k_{\perp}$. At small $k_{\perp}$ and large $\kappa$, momentum broadening in a weakly coupled plasma can be thought of as diffusion in $k_{\perp}$-space, as is the case at all $\kappa$ and all $k_{\perp}$ in a plasma that is strongly coupled at all length scales. At large enough $k_{\perp}$, however, momentum broadening in a weakly coupled plasma is dominated by single, rare, hard, large-angle scattering off point-like quasiparticles. In the large- $k_{\perp}$ regime, the power-law tail of $P\left(k_{\perp}\right)$ is unaffected by resumming $L$-enhanced diagrams precisely because for any given $L$ it arises only at values of $k_{\perp}$ that are so large that $P\left(k_{\perp}\right)$ describes rare single hard scattering. Although rare, these scatterings are more common than in a plasma that is a strongly coupled liquid at all scales, like that in strongly coupled $\mathcal{N}=4 \mathrm{SYM}$ theory, because in such a plasma there are no weakly coupled point-like constituents at any length-scale.

Although there are many reasons not to attempt a quantitative comparison between our calculation and any results from experiment (for example, we applied a leading order calculation valid for $g<1$ at $g \sim 2$; for example, we have not yet included effects of gluon radiation) at a qualitative level it is easy to see what our calculation predicts for $P\left(k_{\perp}\right)$ in the QCD plasma at experimentally relevant temperatures: as long as observables that bias toward short $L$ are not used, we should expect $P\left(k_{\perp}\right)$ to be Gaussian at low $k_{\perp}$ and to have a power-law tail at large $k_{\perp}$. Even if the QCD plasma can be best understood as a strongly coupled fluid without quasiparticles at its natural length scales of order $1 / T$, since QCD is asymptotically free we know that at short distances the strongly coupled quark-gluon plasma is made of weakly coupled quarks and gluons and this means that at high enough $k_{\perp}$ a power-law tail must rise above the Gaussian in $P\left(k_{\perp}\right)$, as in our calculations in which the plasma is assumed to be weakly coupled at all length scales.

What about the jet quenching parameter $\hat{q} \equiv\left\langle k_{\perp}^{2}\right\rangle / L$ ? Our calculation shows that its role is very different in a plasma that is weakly coupled at short distances than in one that is strongly coupled on all length scales. In the latter case, if $P\left(k_{\perp}\right)$ is Gaussian, 
as in the strongly coupled $\mathcal{N}=4$ SYM plasma, then $\hat{q}$ is simply a measure of the width of the Gaussian probability distribution. In some other plasma that is strongly coupled on all length scales, the same would be true even if $P\left(k_{\perp}\right)$ were not precisely Gaussian. On the other hand, if $P\left(k_{\perp}\right) \propto 1 / k_{\perp}^{4}$ at large $k_{\perp}$, as in any plasma that contains weakly coupled constituents at short enough length scales, then $\hat{q}$ is ultraviolet divergent and so is determined almost completely by the power-law tail of the probability distribution $P\left(k_{\perp}\right)$. In this circumstance, $\hat{q}$ knows almost nothing about the (Gaussian) behavior of $P\left(k_{\perp}\right)$ in the regime where almost all of the probability resides. In this circumstance, therefore, $\hat{q}$ tells us almost nothing about the momentum broadening of almost all hard partons. In this circumstance, $\hat{q}$ is almost entirely determined by the improbably small fraction of hard partons that scatter at large angles.

We close with three observations. First, the jet quenching parameter by itself is not sufficient as a characterization of $P\left(k_{\perp}\right)$. Depending on the circumstances, it could be a characterization of the "bulk" of the probability distribution for momentum broadening or it could be telling us only about the power-law "fat tail" of this distribution, in other words only about the physics of those few hard partons that pick up improbably large $k_{\perp}$. Knowing how to interpret what a value of $\hat{q}$ means requires knowing more than $\hat{q}$; it requires further characterization of the shape of $P\left(k_{\perp}\right)$. Second, if at some future time it is possible to use data from heavy ion collision experiments to characterize the shape of $P\left(k_{\perp}\right)$ over the range of $k_{\perp}$ where most of the probability lies, it will take quantitative knowledge of this shape to learn about the properties of the plasma - since we have seen an example in which a very similar shape arises if the plasma is assumed weakly coupled or if the plasma is assumed to be infinitely strongly coupled. Third, the place to look in order to distinguish between these cases qualitatively is large $k_{\perp}$. Just as Rutherford discovered point-like nuclei within what he thought were liquid-like atoms, we can find evidence for the weakly coupled quarks and gluons that we know to be the short length-scale constituents of the strongly coupled plasma produced in heavy ion collision experiments.

It remains to be seen which experimental observables provide access to $P\left(k_{\perp}\right)$ at large $k_{\perp}$. The most direct approach that we are aware of is to analyze events in which an initial hard scattering produces an energetic photon back-to-back with an energetic quark. The photon tells us the initial energy and direction of the quark, and we can then hope to determine the $k_{\perp}$ relative to this initial direction that the quark has picked up as it propagates through the plasma. Figure 15 suggests it would be very interesting to determine whether the quark picks up $k_{\perp} \sim 20 \mathrm{GeV}$ or more a fraction of a percent of the time, even a small fraction of a percent of the time, versus not at all. Seeing rare, but large, momentum kicks would confirm that (as we know) at short enough distance scales quark-gluon plasma is made of quark and gluon quasiparticles. It would then become very interesting to study the intermediate $k_{\perp}$-range in detail, to start to understand how a strongly coupled liquid emerges from an asymptotically free gauge theory. We will be watching with interest as early results on jets and energetic hadrons back-to-back with a single hard photon in heavy ion collisions [10, 13-20] develop, and as the statistics of such measurements improve. 


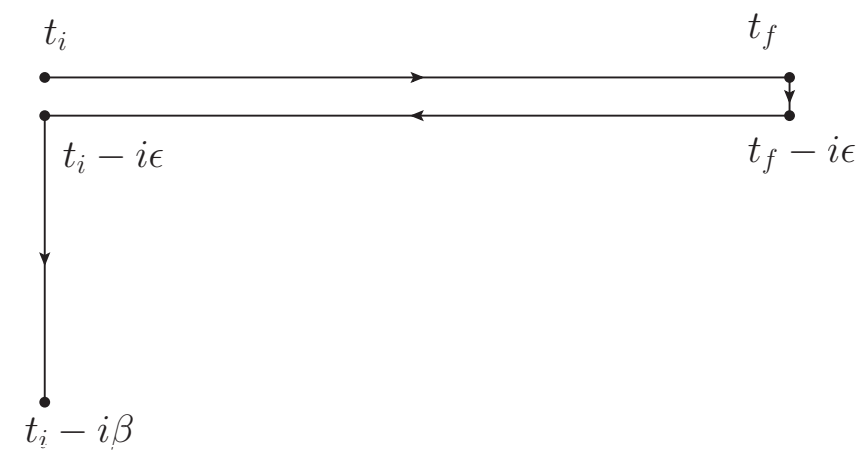

Figure 16. Schwinger-Keldysh contour for $\sigma=\epsilon$.

\section{Acknowledgments}

We are particularly grateful to Chris Lee for many very helpful conversations, in which he taught us about plus distributions and more. We also acknowledge helpful conversations with Andrea Allais, Peter Arnold, Simon Caron-Huot, Iain Stewart, Xin-Nian Wang and Urs A. Wiedemann. This work is supported by U.S. Department of Energy (D.O.E.) under cooperative research agreement DE-FG0205ER41360. The work of F.D. is supported by the Miller Institute for Basic Research in Science, University of California, Berkeley.

\section{A Schwinger-Keldysh formalism}

In this appendix, we briefly review the real-time field theory tools that we use in setting up the formalism in section II and calculating the self-energy in section III. (The Schwinger-Keldysh formalism that we are using is standard. One of its advantages is that by formally doubling the number of degrees of freedom, pinch singularities that otherwise plague real-time quantum field theory are avoided. For a more complete review, see e.g. ref. [94].) We present the discussion for a scalar field theory to avoid excess notation. The discussion for gauge bosons is analogous.

We allow the time coordinate $x^{0}$ to be complex, and we define thermal Green functions

$$
G_{C}\left(x_{1}, x_{2}, \ldots, x_{n}\right)=\left\langle T_{C}\left\{\phi\left(x_{1}\right) \phi\left(x_{2}\right) \ldots \phi\left(x_{n}\right)\right\}\right\rangle,
$$

where the ordering is taken along a path $C$ in the complex plane. For the path $C$ we choose the Schwinger-Keldysh contour in figure 16. In the $t_{i} \rightarrow-\infty$ and $t_{f} \rightarrow \infty$ limit the vertical pieces of $C$ are irrelevant for the calculation of Green functions. Thus it is convenient to label the field $\phi_{i}(x)$, where $i=1$ or $i=2$ depending on where the field is evaluated on $C$ :

$$
\phi_{1}(x)=\phi_{1}\left(x^{0}, \mathbf{x}\right), \quad \phi_{2}(x)=\phi_{2}\left(x^{0}-i \epsilon, \mathbf{x}\right) .
$$

Propagators then become matrices:

$$
D_{i j}(x-y)=\left(\begin{array}{c}
\left\langle T\left\{\phi\left(x^{0}, \mathbf{x}\right) \phi\left(y^{0}, \mathbf{y}\right)\right\}\right\rangle\left\langle\phi\left(y^{0}-i \epsilon, \mathbf{y}\right) \phi\left(x^{0}, \mathbf{x}\right)\right\rangle \\
\left\langle\phi\left(x^{0}-i \epsilon, \mathbf{x}\right) \phi\left(y^{0}, \mathbf{y}\right)\right\rangle\left\langle\tilde{T}\left\{\phi\left(x^{0}, \mathbf{x}\right) \phi\left(y^{0}, \mathbf{y}\right)\right\}\right\rangle
\end{array}\right)
$$


where $T$ and $\tilde{T}$ stand for time ordering and anti-time ordering, respectively. The generating functional for the free theory can be written as

$$
\begin{aligned}
Z_{C}^{\text {free }} & =\mathcal{N} \exp \left[-\frac{1}{2} \int_{-\infty}^{\infty} d^{4} x \int_{-\infty}^{\infty} d^{4} x^{\prime} K\left(x, x^{\prime}\right)\right], \\
K\left(x, x^{\prime}\right) & \equiv J_{i}(x) D_{i j}^{\text {free }}\left(x-x^{\prime}\right) J_{j}\left(x^{\prime}\right)
\end{aligned}
$$

where the normalization factor $\mathcal{N}$ takes into account the multiplicative constant given by the two vertical pieces in $C$. In order to get the thermal Green functions we have to differentiate the above expression with respect to $J_{i}(x)$. For $\epsilon \rightarrow 0$, the Fourier space free propagator matrix reads

$$
\begin{aligned}
D_{11}^{\text {free }}(Q) & =\left[\frac{i}{Q^{2}+i \epsilon}+n\left(q_{0}\right) 2 \pi \delta\left(Q^{2}\right)\right], \\
D_{12}^{\text {free }}(q) & =\left[\theta\left(-q_{0}\right)+n\left(q_{0}\right)\right] 2 \pi \delta\left(Q^{2}\right), \\
D_{21}^{\text {free }}(Q) & =\left[\theta\left(q_{0}\right)+n\left(q_{0}\right)\right] 2 \pi \delta\left(Q^{2}\right), \\
D_{22}^{\text {free }}(q) & =\left[\frac{-i}{Q^{2}-i \epsilon}+n\left(q_{0}\right) 2 \pi \delta\left(Q^{2}\right)\right] .
\end{aligned}
$$

The $\phi_{2}(x)$ field induces a modification of the naive Feynman rules. The propagator has off-diagonal elements, meaning that it mixes the two fields 1 and 2 , whereas the vertices have only one type of field, and they do not induce any mixing. In addition we have a minus sign for any vertex connecting fields of type 2 .

The four components of the propagator matrix in (A.3) are not independent, since

$$
D_{11}(Q)+D_{22}(Q)=D_{12}(Q)+D_{21}(Q)
$$

and it is therefore more convenient to use a different basis including only three independent propagators. We introduce the Keldysh representation

$$
\begin{aligned}
\text { Retarded : } & D_{R}(Q) \equiv D_{11}(Q)-D_{12}(Q), \\
\text { Advanced : } & D_{A}(Q) \equiv D_{11}(Q)-D_{21}(Q), \\
\text { Symmetric : } & D_{S}(Q) \equiv D_{11}(Q)+D_{22}(Q) .
\end{aligned}
$$

From the definitions we can derive the Fourier space expressions for the three free propagators in this new basis.

$$
\begin{aligned}
\text { Retarded : } & D_{R}^{\mathrm{free}}(Q)=\frac{i}{Q^{2}+i \operatorname{sgn}\left(q_{0}\right) \epsilon}, \\
\text { Advanced : } & D_{A}^{\mathrm{free}}(Q)=\frac{i}{Q^{2}-i \operatorname{sgn}\left(q_{0}\right) \epsilon}, \\
\text { Symmetric : } & D_{S}^{\mathrm{free}}(Q)=\left[1+2 f\left(q_{0}\right)\right] 2 \pi \delta\left(Q^{2}\right) .
\end{aligned}
$$

We notice that only the symmetric propagator contains the thermal distribution $n\left(q_{0}\right)$ (either the Bose-Einstein distribution $n_{B}\left(q_{0}\right)$ or the Fermi-Dirac distribution $n_{F}\left(q_{0}\right)$ as appropriate) simplifying the identification of thermal contributions to diagrams. 
We can now introduce the quantity that we calculate in section III, the self-energy matrix $\Pi_{i j}$ which is the building block for the propagator $D_{i j}(Q)$, as described by the Dyson equation

$$
D_{i j}(Q)=D_{i j}^{\mathrm{free}}(Q)+D_{i m}^{\mathrm{free}}(Q)\left(-i \Pi_{m n}(Q)\right) D_{n j}(Q) .
$$

In order to use the Keldysh representation also for the self-energy we define

$$
D_{\alpha}(Q)=D_{\alpha}^{\text {free }}(Q)+D_{\alpha}^{\text {free }}(Q)\left(-i \Pi_{\alpha}(Q)\right) D_{\alpha}(Q),
$$

where $\alpha=R, A, S$. The self-energies $\Pi_{\alpha}(Q)$ introduced above are linear combinations of the self-energies $\Pi_{i j}$. This is shown by plugging the propagators $D_{i j}(Q)$ into the definition (A.10), and identifying

$$
\begin{aligned}
\text { Retarded : } & \Pi_{R}(Q)=\Pi_{11}(Q)+\Pi_{12}(Q), \\
\text { Advanced : } & \Pi_{A}(Q)=\Pi_{11}(Q)+\Pi_{21}(Q), \\
\text { Symmetric : } & \Pi_{S}(Q)=\Pi_{11}(Q)+\Pi_{22}(Q) .
\end{aligned}
$$

\section{B A thin medium}

In this appendix we analyze our results in the case of a "thin medium", by which we mean $L$ short enough such that $\kappa \equiv g^{2} C_{\mathcal{R}} L T /(2 \pi) \ll 1$. In this regime, the resummation of length-enhanced contributions performed in section 2 is not necessary meaning that in this regime the full expression (2.15) reduces to

$$
P\left(k_{\perp}\right) \simeq \int d^{2} x_{\perp} e^{-i k_{\perp} \cdot x_{\perp}}\left[1+\mathcal{W}_{\mathcal{R}}^{(2)}\left(x_{\perp}\right)\right] .
$$

We rewrite this expression in Fourier space, exactly as we did at the end of section 2, and we find

$$
P\left(k_{\perp}\right)=(2 \pi)^{2} \delta^{2}\left(k_{\perp}\right)\left[1-\int \frac{d^{2} q_{\perp}}{(2 \pi)^{2}} P_{\text {thin }}\left(q_{\perp}\right)\right]+P_{\text {thin }}\left(k_{\perp}\right)
$$

where $P_{\text {thin }}\left(k_{\perp}\right)$ is the function defined in eq. (5.2), a definition that we reproduce here for convenience:

$$
P_{\text {thin }}\left(k_{\perp}\right) \equiv \frac{2 \sqrt{2} \pi \kappa}{T} \int \frac{d q^{-}}{2 \pi} D^{>}\left(q^{-}, k_{\perp}\right) .
$$

The probability distribution in eq. (B.2) consists of two parts. First, we see a delta function centered at $k_{\perp}=0$ whose coefficient has two terms, corresponding to no scattering and scattering with no transverse momentum exchange. Second, we see the contribution describing scattering with nonzero final transverse momentum, given just by $P_{\text {thin }}\left(k_{\perp}\right)$. We see that for a thin medium, and for $k_{\perp} \neq 0$, we have $P\left(k_{\perp}\right)=P_{\text {thin }}\left(k_{\perp}\right)$.

Looking at the probability distribution (B.2), we immediately notice that the coefficient of the delta function is not finite. As we have seen in section V.A, $P_{\text {thin }}\left(q_{\perp}\right)$ precisely matches the AGZ distribution (5.5) in the infrared, meaning that it is $\propto q_{\perp}^{-2}$ for $q_{\perp} \ll T$. The integral in the delta function coefficient thus blows up. We shall show that, despite this divergence, the probability distribution in eq. (B.2) is well defined. In order 
to do that we need to introduce the "plus distribution" $[\ldots]_{+}$for a generic function $g(x)$, defined as $[95,96]$

$$
\begin{aligned}
{[\theta(x) g(x)]_{+} } & \equiv \lim _{\beta \rightarrow 0} \frac{d}{d x}[\theta(x-\beta) G(x)], \text { with } \\
G(x) & =\int_{x_{0}}^{x} d x^{\prime} g\left(x^{\prime}\right),
\end{aligned}
$$

satisfying the boundary condition $\int_{0}^{x_{0}} d x[\theta(x) g(x)]_{+}=0$. Here, $x_{0} \neq 0$ when $g(0)=\infty$. Since $P\left(k_{\perp}\right)$ depends only on the absolute value of transverse momentum, it is convenient to use the one-dimensional probability distributions $\tilde{P}\left(k_{\perp}\right) \equiv 2 \pi k_{\perp} P\left(k_{\perp}\right)$ and $\tilde{P}_{\text {thin }}\left(k_{\perp}\right) \equiv 2 \pi k_{\perp} P_{\text {thin }}\left(k_{\perp}\right)$. Then (B.2) becomes

$$
\tilde{P}\left(k_{\perp}\right)=(2 \pi)^{2} \delta\left(k_{\perp}\right)+\tilde{P}_{\text {thin }}\left(k_{\perp}\right)-\delta\left(k_{\perp}\right) \int_{0}^{\infty} d q_{\perp} \tilde{P}_{\text {thin }}\left(q_{\perp}\right),
$$

where the normalization now reads $\int_{0}^{\infty} d k_{\perp} \tilde{P}\left(k_{\perp}\right)=(2 \pi)^{2}$.

We can apply the plus distribution prescription to extract the divergent term from $\tilde{P}_{\text {thin }}\left(k_{\perp}\right)$, obtaining

$$
\left[\theta\left(k_{\perp}\right) \tilde{P}_{\text {thin }}\left(k_{\perp}\right)\right]_{+}=\lim _{\beta \rightarrow 0}\left[\delta\left(k_{\perp}-\beta\right) \int_{k_{\perp 0}}^{k_{\perp}} d q_{\perp} \tilde{P}_{\text {thin }}\left(q_{\perp}\right)+\theta\left(k_{\perp}-\beta\right) \tilde{P}_{\text {thin }}\left(k_{\perp}\right)\right] .
$$

After we take the $\beta \rightarrow 0$ limit, and noting that $k_{\perp}$ is always positive, the above result takes the form

$$
\left[\tilde{P}_{\text {thin }}\left(k_{\perp}\right)\right]_{+}=\tilde{P}_{\text {thin }}\left(k_{\perp}\right)-\delta\left(k_{\perp}\right) \int_{k_{\perp}}^{k_{\perp 0}} d q_{\perp} \tilde{P}_{\text {thin }}\left(q_{\perp}\right) .
$$

This in turns implies the following facts:

- $\int_{0}^{k_{\perp 0}} d k_{\perp}\left[\tilde{P}_{\text {thin }}\left(k_{\perp}\right)\right]_{+}=0$

- $\left[\tilde{P}_{\text {thin }}\left(k_{\perp}\right)\right]_{+}=\tilde{P}_{\text {thin }}\left(k_{\perp}\right)$ for $k_{\perp}>0$.

Combining (B.6) and (B.4), we obtain

$$
\tilde{P}\left(k_{\perp}\right)=\delta\left(k_{\perp}\right)\left((2 \pi)^{2}-\int_{k_{\perp 0}}^{\infty} d q_{\perp} \tilde{P}_{\text {thin }}\left(q_{\perp}\right)\right)+\left[\tilde{P}_{\text {thin }}\left(k_{\perp}\right)\right]_{+},
$$

where $k_{\perp 0}$ is a free parameter in the range $0<k_{\perp 0}<\infty$.

If we switch back to the two dimensional distributions, drop the tildes, and keep in mind that the plus distribution $\left[P\left(k_{\perp}\right)\right]_{+}$is formally the one dimensional plus distribution divided by $2 \pi k_{\perp}$, we find

$$
P\left(k_{\perp}\right)=\delta^{2}\left(k_{\perp}\right) g\left(k_{\perp 0}\right)+\left[P_{\text {thin }}\left(k_{\perp}\right)\right]_{+}^{k_{\perp 0}},
$$

where the factor $g\left(k_{\perp 0}\right)$ reads

$$
g\left(k_{\perp 0}\right)=(2 \pi)^{2}-2 \pi \int_{k_{\perp 0}}^{\infty} d q_{\perp} q_{\perp} P_{\text {thin }}\left(q_{\perp}\right) .
$$


For $k_{\perp 0}>0$, the coefficient of the delta function in eq. (B.8) is finite and positive, and the last term in eq. (B.8) is the probability distribution with its value at $k_{\perp}=0$ having been subtracted. Even though both terms depend on $k_{\perp 0}$, the $k_{\perp 0}$ dependence cancels when we sum the two terms. In conclusion, the probability distribution $P\left(k_{\perp}\right)$ in the thin medium limit $g^{2} C_{\mathcal{R}} L T \ll 1$ is well defined, and the probability for no scattering plus that for scattering with no transverse momentum exchange is finite and positive, as it must be.

\section{Exponentiation of $\mathcal{W}_{\mathcal{R}}^{(2)}$}

In this appendix, we show that by summing over all disconnected diagrams which involve only $\mathcal{W}_{\mathcal{R}}^{(2)}$, we obtain precisely the exponential (2.16) that we have used in (2.15). To get the lowest power in $g$ for a given power of $L$, there is no need to include any connected diagrams other than $\mathcal{W}_{\mathcal{R}}^{(2)}$ in the sum. As discussed in the main text, if we ignore any factors of $g$ that come from within the gluon propagators $\mathcal{W}_{\mathcal{R}}^{(2)}$ is of order $g^{2} L$, while any other connected diagrams give rise to $g^{n} L$ with $n>2$ and thus are of higher order. For example, $\mathcal{W}_{\mathcal{R}}^{(3)}$ is of order $g^{3} L$, while the cross diagram in figure 4 will give a factor $g^{4} L$.

To obtain the $n$-th order term in (2.16), we consider the expansion of $\mathcal{W}_{\mathcal{R}}$ at order $j=2 n$. For illustration here we demonstrate explicitly the exponentiation of $D^{>}\left(y^{-}, x_{\perp}\right)$ in (2.12) which comes from contractions of gluons with ends on different Wilson lines. The story for $D^{>}\left(y^{-}, 0_{\perp}\right)$ (gluons with both ends on the same Wilson line) and the cross terms between $D^{>}\left(y^{-}, 0_{\perp}\right)$ and $D^{>}\left(y^{-}, x_{\perp}\right)$ are similar and we leave it to the reader that they also exponentiate. In the notation of eq. (2.4), we need only consider the term $W_{\mathcal{R}}^{(n, n)}$ in $\mathcal{W}_{\mathcal{R}}^{(2 n)}$, which can be written as

$$
\begin{aligned}
W_{\mathcal{R}}^{(n, n)}= & \frac{g^{n}}{d(\mathcal{R})} \int_{x_{1}^{-}<x_{2}^{-}<\cdots x_{n}^{-}} d x_{n}^{-} \ldots d x_{z_{1}^{-}<z_{2}^{-}<\cdots z_{n}^{-}} d z_{n}^{-} \ldots d z_{n}^{-} \\
& \times\left\langle\operatorname{Tr}\left[A_{\mathcal{R}}^{+}\left(x_{1}^{-}, x_{\perp}\right) \ldots A_{\mathcal{R}}^{+}\left(x_{n}^{-}, x_{\perp}\right) A_{\mathcal{R}}^{+}\left(z_{n}^{-}, 0_{\perp}\right) \ldots A_{\mathcal{R}}^{+}\left(z_{1}^{-}, 0_{\perp}\right)\right]\right\rangle
\end{aligned}
$$

where we have denoted the coordinates on the two different Wilson lines by $x_{i}^{-}$and $z_{i}^{-}$ respectively and made the path ordering explicit. There are many different contractions in (C.1). The one which is relevant for our purposes here is the one in which the gluon at $x_{i}^{-}$is contracted with the gluon at $z_{i}^{-}$, for each of $i=1,2, \cdots n$. Other contractions will either give rise to cross terms between $D^{>}\left(y^{-}, x_{\perp}\right)$ and $D^{>}\left(y^{-}, 0_{\perp}\right)$ or create cross diagrams like that in figure 4 which are of higher order. We then find that

$$
\begin{aligned}
W_{\mathcal{R}}^{(n, n)} & =\left(g^{2} C_{\mathcal{R}}\right)^{n} \int_{x_{1}^{-}<x_{2}^{-}<\cdots x_{n}^{-}} d x_{n}^{-} \ldots d x_{z_{1}^{-}<z_{2}^{-}<\cdots z_{n}^{-}} d z_{n}^{-} \prod_{i=1}^{n} D^{>}\left(x_{i}^{-}-z_{i}^{-}, x_{\perp}\right) \\
& =\frac{1}{n !}\left(g^{2} C_{\mathcal{R}} L^{-} \int d y^{-} D^{>}\left(y^{-}, x_{\perp}\right)\right)^{n} .
\end{aligned}
$$

In the last step we have changed the integration over each pair of integration variables $\left(x_{i}^{-}, z_{i}^{-}\right)$to an integration over $Y_{i}^{-}=\frac{x_{i}^{-}+z_{i}^{-}}{2}$ and $y_{i}^{-}=x_{i}^{-}-z_{i}^{-}$. The integrations over $y_{i}^{-}$are unconstrained, while the integrations over $Y_{i}^{-}$, which satisfy the ordering $Y_{1}^{-}<Y_{2}^{-}<\cdots Y_{n}^{-}$, give $\left(L^{-}\right)^{n} / n$ !. We have proved (2.15). The generalization of the proof 
of the exponentiation of $\mathcal{W}_{\mathcal{R}}^{(2)}$ that we have presented here to a proof of the exponentiation of the sum of all connected diagrams is straightforward.

\section{Boltzmann equation approach}

In this appendix, we provide an explicit alternative derivation of the expression (2.15) for the probability distribution $P\left(k_{\perp}\right)$ by deriving and solving an appropriate Boltzmann equation for momentum broadening. The argument can easily be generalized to obtain the more general result (2.14) that goes beyond leading order in weak coupling. In this appendix, it is useful to make the $L$-dependence of the probability distribution explicit by denoting it as $P\left(k_{\perp}, L\right)$.

Let us consider the hard parton after it has propagated for a distance $L$ in the hot and dense medium. $P\left(k_{\perp}, L\right)$ is the probability distribution for its transverse momentum $k_{\perp}$. We want to relate $P\left(k_{\perp}, L\right)$ to $P\left(k_{\perp}, L+\Delta L\right)$, the probability distribution after the parton has traveled a further distance $\Delta L$. In the $\Delta L \ll L$ limit, modifications to $P\left(k_{\perp}, L\right)$ are only caused by a single scattering event. We then introduce the differential collision kernel $C\left(q_{\perp}\right)$, defined as the differential rate for momentum broadening due to elastic collisions

$$
C\left(q_{\perp}\right) \equiv \frac{d^{2} \Gamma}{d q_{\perp}^{2}}
$$

in terms of which the relation between the probability distributions at $L$ and $L+\Delta L$ can be written as

$$
P\left(k_{\perp}, L+\Delta L\right)=P\left(k_{\perp}, L\right)\left[1-\Delta L \int \frac{d^{2} q_{\perp}}{(2 \pi)^{2}} C\left(q_{\perp}\right)\right]+\Delta L \int \frac{d^{2} q_{\perp}}{(2 \pi)^{2}} C\left(q_{\perp}\right) P\left(k_{\perp}-q_{\perp}, L\right) .
$$

The first line describes the probability that between $L$ and $L+\Delta L$ there is no further momentum transfer, and the probability distribution is not affected. The second line takes into account the possibility that the transverse momentum $k_{\perp}$ at $L+\Delta L$ arises after the hard parton picks up an additional transverse momentum $q_{\perp}$ in a scattering event that occurs between $L$ and $L+\Delta L$. The Boltzmann equation for transverse momentum broadening is obtained by taking the $\Delta L \rightarrow 0$ limit in eq. (D.2), yielding

$$
\frac{d P\left(k_{\perp}, L\right)}{d L}=\int \frac{d^{2} q_{\perp}}{(2 \pi)^{2}} C\left(q_{\perp}\right)\left[P\left(k_{\perp}-q_{\perp}, L\right)-P\left(k_{\perp}, L\right)\right] .
$$

Solving the Boltzmann equation (D.3) is simpler in coordinate space, where it reads

$$
\frac{d P\left(x_{\perp}, L\right)}{d L}=-v\left(x_{\perp}\right) P\left(x_{\perp}, L\right),
$$

with

$$
v\left(x_{\perp}\right) \equiv \int \frac{d^{2} q_{\perp}}{(2 \pi)^{2}}\left[1-e^{i q_{\perp} x_{\perp}}\right] C\left(q_{\perp}\right),
$$

a quantity often referred to as the dipole cross-section in the literature. The solution of eq. (D.4) is just an exponential function, and upon performing the Fourier transform back 
to momentum space we find

$$
P\left(k_{\perp}, L\right)=\int d^{2} x_{\perp} e^{i k_{\perp} x_{\perp}} e^{-v\left(x_{\perp}\right) L} .
$$

This connection between multiple scattering and the dipole cross section was recognized in refs. [22-25]. In many calculations, however, the dipole cross-section was approximated as $v\left(x_{\perp}\right) \approx C x_{\perp}^{2}$, resulting in a probability distribution that is Gaussian in $k_{\perp}$. As illustrated in figure 12, we find that this is a good approximation at small $k_{\perp}$ but not at large $k_{\perp}$.

Next, we present the explicit relationship between the analysis of this appendix in terms of a Boltzmann equation and the analysis we follow throughout all other sections of this paper. The building block for deriving the Boltzmann equation was the differential elastic collision kernel $C\left(k_{\perp}\right)$ defined in eq. (D.1). With a view toward making contact with results obtained in previous literature via the Boltzmann equation approach, we begin by including only elastic collisions in which one gluon exchanged. In the framework of the rest of this paper, this is obtained from the Wilson line diagrams in figure 1 with only one gluon propagator, as in figure 2 . We have denoted this contribution by $P_{\text {thin }}\left(k_{\perp}, L\right)$, defined in eq. (5.2). The connection between $P_{\text {thin }}\left(k_{\perp}, L\right)$ and $C\left(k_{\perp}\right)$ reads

$$
P_{\text {thin }}\left(k_{\perp}, L\right)=C\left(k_{\perp}\right) L
$$

With this identification in mind, we revisit the expression for $\mathcal{W}_{\mathcal{R}}^{(2)}$ found in eq. (2.18), and we recognize

$$
\mathcal{W}_{\mathcal{R}}^{(2)}\left(x_{\perp}\right)=-\int \frac{d^{2} q_{\perp}}{(2 \pi)^{2}}\left[1-e^{i q_{\perp} \cdot x_{\perp}}\right] P_{\text {thin }}\left(q_{\perp}, L\right)=-v\left(x_{\perp}\right) L .
$$

The solution to the Boltzmann equation found in eq. (D.6), with $v\left(x_{\perp}\right)$ connected to $\mathcal{W}_{\mathcal{R}}^{(2)}$ as in eq. (D.8), is therefore identical to the expression in eq. (2.15) obtained by resumming length-enhanced diagrams.

The manipulations in this appendix are completely general in the sense that they do not rely on any specific form for the collision kernel $C\left(k_{\perp}\right)$ in eq. (D.1). If we were to include contributions to $C\left(k_{\perp}\right)$ that are higher order in the coupling, solving the Boltzmann equation would resum these higher order effects as needed when $L$ is not small. If we were to include all contributions to $C\left(k_{\perp}\right)$ to arbitrarily high order in the coupling, we would recover our general result (2.14).

It has long been understood (in the QCD context, since refs. [23, 81, 82]; in the context of electrons propagating through ordinary matter, at least since refs. [97, 98]) that if $L$ is long enough that multiple scattering is important, the dipole cross section can to a degree be approximated as $v\left(x_{\perp}\right) \propto x_{\perp}^{2}$, corresponding to a probability distribution for transverse momentum broadening (D.6) that is Gaussian in $k_{\perp}$. It was also understood by all these authors that, at large enough $k_{\perp}, P\left(k_{\perp}\right)$ must have a power-law tail corresponding to Rutherford scattering off a single point-like scatterer. In the QCD context, this was demonstrated in a model context by Wiedemann and Gyulassy in appendix A of ref. [81], where they showed that in the Gyulassy-Wang model [21] (in which the medium consists of 
static scattering centers which the hard parton sees as Debye-screened Yukawa potentials) the dipole cross section takes the form $v\left(x_{\perp}\right) \propto x_{\perp}^{2} \log \left(R^{2} / x_{\perp}^{2}\right)$ at small $\left|x_{\perp}\right|$ for some constant $R$. (This form was known earlier in a different context $[99,100]$.) Evaluating (D.6), we see that this form for $v\left(x_{\perp}\right)$ corresponds to $P\left(k_{\perp}\right) \propto 1 / k_{\perp}^{4}$ at large $k_{\perp}$.

In the calculation we have presented in this paper, we need make no model assumptions about the nature of the medium. It is a weakly coupled quantum field theoretical plasma, as in QCD in thermal equilbrium at asymptotically high temperatures. And, as illustrated in figure 12, when $L$ is large enough that multiple scattering is important we find by direct calculation both the Gaussian behavior at small $k_{\perp}$ and the $1 / k_{\perp}^{4}$ behavior at large $k_{\perp}$.

\section{E A tensor basis for the self-energy}

In this appendix we define the projectors used to expand the retarded self-energy in tensor components in section III and describe their properties. The presence of the thermal bath breaks Lorentz invariance by specifying a preferred frame in which the medium is at rest. We shall work in the medium rest frame, in which its four-velocity $U^{\mu}$ is $U^{\mu}=(1,0)$, but in this appendix we keep $U^{\mu}$ unspecified, defining these projectors for any frame. It is convenient to introduce another four-vector $N^{\mu}$ by projecting the four-velocity $U^{\mu}$ onto the direction orthogonal to the external momentum $Q^{\mu}$ :

$$
\begin{aligned}
& N^{\mu} \equiv P^{\mu \nu} U_{\nu}=U^{\mu}-\frac{Q \cdot U}{Q^{2}} Q^{\mu}, \\
& P_{\mu \nu}=g_{\mu \nu}-\frac{Q_{\mu} Q_{\nu}}{Q^{2}}, \quad Q \cdot N=0 .
\end{aligned}
$$

The self-energy is a symmetric rank-2 tensor. It can therefore be expressed as a linear combination of the following tensors:

$$
\begin{aligned}
P_{\mu \nu}^{T} & =-g_{\mu \nu}+\frac{Q_{\mu} Q_{\nu}}{Q^{2}}+\frac{N_{\mu} N_{\nu}}{N^{2}}, & P_{\mu \nu}^{L} & =-\frac{N_{\mu} N_{\nu}}{N^{2}}, \\
J_{\mu \nu} & =\frac{Q_{\mu} N_{\nu}+N_{\mu} Q_{\nu}}{\sqrt{-2 N^{2} Q^{2}}}, & K_{\mu \nu} & =\frac{Q_{\mu} Q_{\nu}}{Q^{2}} .
\end{aligned}
$$

It is useful to build a multiplication table for the tensor basis, keeping in mind that the tensors in (E.2) are symmetric in their Lorentz indices. We find

$$
\begin{aligned}
P^{T} \cdot P^{T} & =-P^{T}, & P^{L} \cdot P^{L} & =-P^{L}, \\
J \cdot J & =\frac{1}{2}\left(P^{L}-K\right), & K \cdot K & =K, \\
P^{T} \cdot P^{L} & =0, & P^{T} \cdot J & =0, \\
P^{T} \cdot K & =0, & P^{L} \cdot K & =0, \\
\operatorname{Tr}\left(P^{L} \cdot J\right) & =0 & \operatorname{Tr}(J \cdot K) & =0 .
\end{aligned}
$$

\section{F Resumming AGZ}

As we noted in section V.A, some years ago Aurenche, Gelis and Zaraket derived an analytic expression (5.5) for $P_{\text {thin }}\left(k_{\perp}\right)$ that is valid in the IR region, $k_{\perp} \ll T$, but not in the UV. 
In this appendix we pursue the exercise of assuming that $P_{\text {thin }}\left(k_{\perp}\right)=P_{\text {thin }}^{\mathrm{AGZ}}\left(k_{\perp}\right)$ at all $k_{\perp}$, not just in the IR, and then resumming $L$-enhanced diagrams to obtain an expression for $P\left(k_{\perp}\right)$ that we shall denote by $P^{\mathrm{AGZ}}\left(k_{\perp}\right)$. Even though $P_{\text {thin }}^{\mathrm{AGZ}}\left(k_{\perp}\right)$ is not in fact correct beyond the IR, there are two reasons to pursue this exercise. First, we anticipate that the $P^{\mathrm{AGZ}}\left(k_{\perp}\right)$ we obtain by resumming the AGZ expression will agree with our complete result at small enough $k_{\perp}$. This makes sense on physical grounds, as we have argued in section 5.2. Here we confirm this expectation by explicit calculation. And, second, starting with the AGZ expression for $P_{\text {thin }}$ allows us to do almost all of the calculation analytically. This makes it of value as a benchmark, even though the result is not valid beyond the IR. And, because the calculation is valid in the IR it will allow us to gain an analytic understanding of the $\kappa$-dependence of the low- $k_{\perp}$ behavior of $P\left(k_{\perp}\right)$.

In order to resum $L$-enhanced diagrams upon assuming that $P_{\text {thin }}\left(k_{\perp}\right)=P_{\text {thin }}^{\mathrm{AGZ}}\left(k_{\perp}\right)$ we proceed as follows. We first note from (2.19) and (5.2) that

$$
\mathcal{W}_{\mathcal{R}}^{(2)}\left(x_{\perp}\right)=-\int \frac{d^{2} q_{\perp}}{(2 \pi)^{2}}\left(1-e^{i q_{\perp} \cdot x_{\perp}}\right) P_{\text {thin }}\left(q_{\perp}\right),
$$

regardless of the form of $P_{\text {thin }}$. In the case where $P_{\text {thin }}=P_{\text {thin }}^{\mathrm{AGZ}}$, given in (5.5), the integral in (F.1) can be done analytically, yielding

$$
\mathcal{W}_{\mathcal{R}}^{(2)}\left(x_{\perp}\right)=\mathcal{W}_{\mathcal{R}}^{(2), \mathrm{AGZ}}\left(x_{\perp}\right) \equiv-\kappa\left[\gamma+\log \left(\frac{x}{2}\right)+K_{0}(x)\right]
$$

where we have defined $x \equiv x_{\perp} m_{D}$ and where $K_{0}(x)$ is the modified Bessel function of the second kind and $\gamma \approx 0.577$ is the Euler gamma constant. Note that $\mathcal{W}_{\mathcal{R}}^{(2)}(x) \rightarrow$ 0 as $x \rightarrow 0$ since $K_{0}(x) \rightarrow-\gamma-\log (x / 2)$ as $x \rightarrow 0$. The dipole cross-section $v\left(x_{\perp}\right)$ corresponding to (F.2) (see appendix D and in particular (D.8)) is very similar to that found in eq. (A11) of ref. [81] for the case of the Gyulassy-Wang model where the medium consists of static Debye-screened scattering centers. This similarity is not surprising: when Fourier transformed, the screened Yukawa potentials in the Gyulassy-Wang model are similar to $P_{\text {thin }}^{A G Z}\left(k_{\perp}\right)$ of eq. (5.5), and in particular they agree at large $k_{\perp}$ where multiple scattering is not important. We therefore find that (F.2) and (A11) of ref. [81] agree at small $x_{\perp}$.

Next, we must substitute (F.2) into (2.15) in order to obtain $P^{\mathrm{AGZ}}\left(k_{\perp}\right)$. The angular integral in (2.15) can be performed analytically, yielding the full, $L$-resummed, probability distribution

$$
P\left(k_{\perp}\right)=P^{A G Z}\left(k_{\perp}\right) \equiv \frac{2 \pi}{m_{D}^{2}} \int_{0}^{\infty} d x x J_{0}\left(\frac{k_{\perp} x}{m_{D}}\right) \times \exp \left[-\kappa\left(\gamma+\log (x / 2)+K_{0}(x)\right)\right]
$$

in which only a single integral remains to be evaluated numerically.

In figure 17, we plot the result (F.3) for the $L$-resummed AGZ distribution for various values of $\kappa$. In the middle and lower panels, which are analogous to the middle and lower panels of figure 10, we show explicitly that the $L$-resummed AGZ distribution agrees with our full result in the IR, as anticipated. This confirms for us that when we inspect the $\kappa$-dependence of $P^{\mathrm{AGZ}}\left(k_{\perp}\right)$ as we do in the top panel, we can trust that at small $k_{\perp}$ these results agree quantitatively with our full results. The first thing that we see in the top 

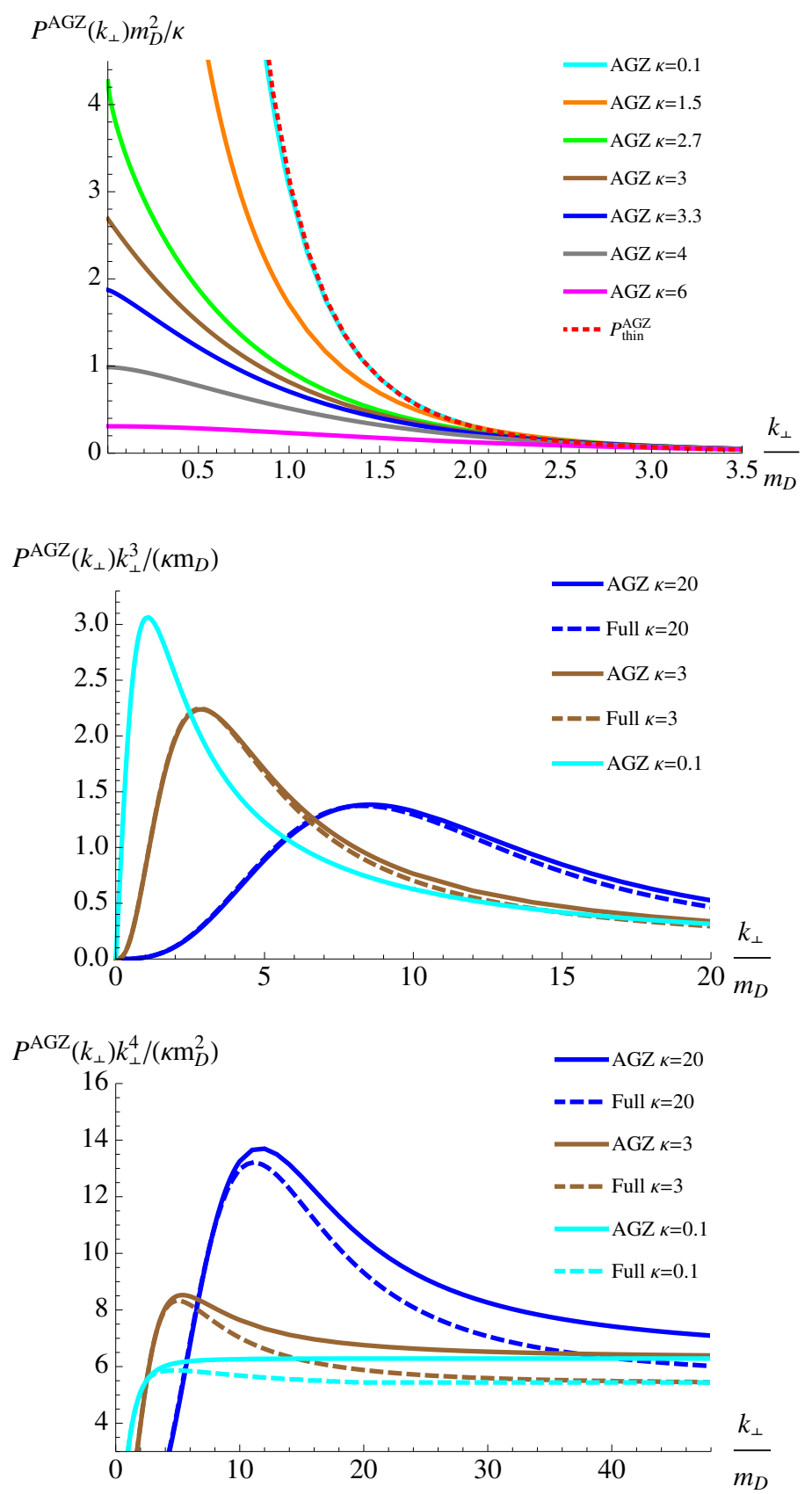

Figure 17. The solid curves in the top panel show the $L$-resummed $P^{\mathrm{AGZ}}\left(k_{\perp}\right)$ for (top to bottom) $\kappa=0.1,1.5,2.7,3,3.3,4$ and 6 . The dashed curve shows $P_{\text {thin }}^{\mathrm{AGZ}}\left(k_{\perp}\right)$; this curve is $\kappa$-independent because we have divided by $\kappa$. We see the $k_{\perp} \rightarrow 0$ behavior of $P^{A G Z}\left(k_{\perp}\right)$ changing as $\kappa$ increases. In the middle and lower panels, both $P^{\mathrm{AGZ}}\left(k_{\perp}\right)$ (solid curves) and our full results for $P\left(k_{\perp}\right)$ from the middle and lower panels of figure 9 (dashed curves) are plotted, multiplied by $k_{\perp}^{3}$ and $k_{\perp}^{4}$. We see that, as expected, the $L$-resummed AGZ distribution agrees with our full result in the IR, but not in the UV. All plots are for $g=0.1$. 
panel is that at $\kappa=0.1$ the $L$-resummed result agrees with that for a thin medium, namely $P_{\text {thin }}^{\mathrm{AGZ}}\left(k_{\perp}\right)$, since when the medium is thin there is no need to resum $L$-enhanced diagrams. Next, by plotting (F.3) for varying $\kappa$ as in this panel we discover that for $\kappa<2$, the resummed result $P^{\mathrm{AGZ}}\left(k_{\perp}\right)$ diverges as $\kappa \rightarrow 0$ while for $\kappa \geq 2$, the resummed result is finite in this limit. Careful inspection of the curves with $\kappa>2$ shows that for $\kappa=3$ the probability $P^{\mathrm{AGZ}}\left(k_{\perp}\right)$ is linear in $k_{\perp}$ as $k_{\perp} \rightarrow 0$, for $2<\kappa<3$ it grows faster than linear in this limit, for $3<\kappa<4$ it grows slower than linear in this limit, and for all $\kappa \geq 4$ the curve is quadratic in $k_{\perp}$ at small $k_{\perp}$. We find the same nontrivial $\kappa$-dependence of our full results at small $k_{\perp}$. The advantage of reproducing these results in the $L$-resummed AGZ distribution (F.3) is that in this simpler setting we can understand them all analytically, as we now explain.

The first step to understanding the $\kappa$-dependence of (F.3) at small $k_{\perp}$ is to divide the $x$-integration in (F.3) into an integral from $x=0$ to some $x=x_{0}$ with $x_{0}$ chosen to be very much greater than 1 , and an integral from $x=x_{0}$ to $x=\infty$. The integral over $0<x<x_{0}$ yields a result that is analytic as $k_{\perp} \rightarrow 0$, whose leading small- $k_{\perp}$ dependence includes a constant term and a term quadratic in $k_{\perp}$. The only place where divergent, or other nonanalytic, behavior can arise as $k_{\perp} \rightarrow 0$ is from the integral over $x_{0}<x<\infty$. In the $k_{\perp} \rightarrow 0$ limit, this integral can be evaluated analytically in terms of generalized hypergeometric functions, whose asymptotic behavior determines that as $k_{\perp} \rightarrow 0$ the probability distribution $P^{\mathrm{AGZ}}\left(k_{\perp}\right)$ must include a term with the form

$$
-\frac{2 \pi}{m_{D}^{\kappa}}\left(2 e^{-\gamma}\right)^{\kappa} f(\kappa) k_{\perp}^{\kappa-2}
$$

with

$$
f(\kappa) \equiv \frac{\kappa}{2^{\kappa}} \frac{\Gamma\left(-\frac{\kappa}{2}\right)}{\Gamma\left(\frac{\kappa}{2}\right)}
$$

for $\kappa \neq 2,4,6, \ldots$ and

$$
f(\kappa) \equiv \frac{4}{4^{\kappa / 2}} \frac{(-1)^{\kappa / 2}}{\Gamma\left(\frac{\kappa}{2}\right)^{2}}\left(\frac{1}{\kappa}+\psi\left(\frac{\kappa}{2}\right)\right),
$$

for $\kappa=4,6,8, \ldots$, where $\Gamma(n)$ and $\psi(n)$ are gamma and digamma functions respectively. The $k_{\perp}$-dependence of this analytic result explains the $k_{\perp} \rightarrow 0$ behavior of all the curves in the top panel of figure 17. For $\kappa>4$, the term (F.4) is subleading relative to the regular $k_{\perp}^{2}$-dependent term at small $k_{\perp}$ and so, for $\kappa \geq 4, P^{\mathrm{AGZ}}\left(k_{\perp}\right)$ is quadratic at small $k_{\perp}$. For $\kappa<4$, (F.4) dominates as $k_{\perp} \rightarrow 0$. And, it is linear in $k_{\perp}$ for $\kappa=3$, grows nonanalytically and slower (faster) than linear for $3<\kappa<4(2<\kappa<3)$ and diverges for $\kappa<2$. So, we have a complete analytic understanding of the nontrivial $\kappa$-dependence of $P^{\mathrm{AGZ}}\left(k_{\perp}\right)$ as $k_{\perp} \rightarrow 0$, and consequently of the same nontrivial $\kappa$-dependence of $P\left(k_{\perp}\right)$ that we described in section 5.2.

We saw in section 5.2, in particular in figure 12, that for large enough $\kappa$ the probability distribution $P\left(k_{\perp}\right)$ is well-approximated as a Gaussian at values of $k_{\perp}$ that are not too large. Given how similar $P^{\mathrm{AGZ}}\left(k_{\perp}\right)$ and $P\left(k_{\perp}\right)$ are at small $k_{\perp}$, see figure 17 , we can conclude that $P^{\mathrm{AGZ}}\left(k_{\perp}\right)$ is also well-approximated as a Gaussian at small values of $k_{\perp}$. Because we have an (almost) analytic understanding of $P^{\mathrm{AGZ}}$ in the form (F.3) we can 
now obtain an (almost) analytic expression for the width of the Gaussians in figure 12 that describe $P\left(k_{\perp}\right)$ at not too large $k_{\perp}$.

In order to find the Gaussian approximation $P^{\mathrm{AGZ}}\left(k_{\perp}\right) \simeq A_{\mathrm{AGZ}} \exp \left(-a_{\mathrm{AGZ}} k_{\perp}^{2}\right)$ to $P^{\mathrm{AGZ}}\left(k_{\perp}\right)$ at low $k_{\perp}$, we Taylor expand the logarithm of $P^{\mathrm{AGZ}}\left(k_{\perp}\right)$. Recall that $P^{\mathrm{AGZ}}\left(k_{\perp}\right)$ is given by (F.3), which we obtained upon resumming length-enhanced diagrams as is necessary at large $\kappa$. We write the expansion as

$$
\log P^{\mathrm{AGZ}}\left(k_{\perp}\right)=\log A_{\mathrm{AGZ}}-a_{\mathrm{AGZ}} k_{\perp}^{2}+\ldots
$$

and find

$$
\begin{aligned}
A_{\mathrm{AGZ}} & =\int_{0}^{\infty} d x b_{\mathrm{AGZ}}(x) \\
a_{\mathrm{AGZ}} & =\frac{1}{4 A_{\mathrm{AGZ}} m_{D}^{2}} \int_{0}^{\infty} d x x^{2} b_{\mathrm{AGZ}}(x),
\end{aligned}
$$

where we have defined

$$
b_{\mathrm{AGZ}}(x) \equiv \frac{2 \pi}{m_{D}^{2}} x \exp \left[-\kappa\left(\gamma+\log (x / 2)+K_{0}(x)\right)\right] .
$$

The expression for $a_{\mathrm{AGZ}}$ in (F.8) can be rewritten as

$$
a_{\mathrm{AGZ}}=\frac{1}{4 m_{D}^{2}} \frac{\int_{0}^{\infty} d x x^{2} b_{\mathrm{AGZ}}(x)}{\int_{0}^{\infty} d x b_{\mathrm{AGZ}}(x)},
$$

from which we notice that any $x$-independent overall factor in $b_{\mathrm{AGZ}}(x)$ does not affect the final result for $a_{\mathrm{AGZ}}$, i.e. for the width of the Gaussian. We can therefore simplify the expression for $a_{\mathrm{AGZ}}$ further, obtaining

$$
a_{\mathrm{AGZ}} m_{D}^{2}=\frac{1}{4} \frac{\int_{0}^{\infty} d x x^{3-\kappa} \exp \left[-\kappa K_{0}(x)\right]}{\int_{0}^{\infty} d x x^{1-\kappa} \exp \left[-\kappa K_{0}(x)\right]},
$$

which is eq. (5.9).

\section{G Simple formula to compute $\hat{q}$}

In this appendix, we derive the simple expression (5.11) for the jet quenching parameter $\hat{q}$, valid for any probability distribution of the form (2.15). We start from the definition of $\hat{q}$ in eq. (5.10) which, with (2.15), reads

$$
\hat{q}=\frac{1}{L} \int \frac{d^{2} k_{\perp}}{(2 \pi)^{2}} k_{\perp}^{2} \int d^{2} x_{\perp} e^{-i k_{\perp} x_{\perp}} e^{\mathcal{W}_{\mathcal{R}}^{(2)}}
$$

By performing the integration over $d^{2} k_{\perp}$, we obtain the two dimensional Laplace operator $\nabla^{2}$ in the transverse plane, acting on a delta function:

$$
\hat{q}=-\frac{1}{L} \int d^{2} x_{\perp}\left[\nabla^{2} \delta^{2}\left(\vec{x}_{\perp}\right)\right] e^{\mathcal{W}_{\mathcal{R}}^{(2)}\left(x_{\perp}\right)} .
$$


We can then integrate the above equation by parts, drop the vanishing boundary terms in our notation, and express $\hat{q}$ as

$$
\hat{q}=-\frac{1}{L} \int d^{2} x_{\perp} \delta^{2}\left(x_{\perp}\right) \nabla^{2} e^{\mathcal{W}_{\mathcal{R}}^{(2)}}=-\left.\frac{1}{L} \nabla^{2} e^{\mathcal{W}_{\mathcal{R}}^{(2)}}\right|_{x_{\perp}=0} .
$$

We can further simplify this expression. By explicit calculation, the Laplace operator acting on the exponential reads

$$
\nabla^{2} e^{\mathcal{W}_{\mathcal{R}}^{(2)}}=e^{\mathcal{W}_{\mathcal{R}}^{(2)}}\left[\nabla^{2} \mathcal{W}_{\mathcal{R}}^{(2)}+\left(\partial_{x} \mathcal{W}_{\mathcal{R}}^{(2)}\right)^{2}+\left(\partial_{y} \mathcal{W}_{\mathcal{R}}^{(2)}\right)^{2}\right]
$$

where we have written the vector in the perpendicular plane as $x_{\perp}=(x, y)$. The last two terms vanish once they are evaluated at $x_{\perp}=0$, since the medium is isotropic in the transverse plane, and we therefore find

$$
\hat{q}=-\left.\frac{1}{L} \nabla^{2} \mathcal{W}_{\mathcal{R}}^{(2)}\right|_{x_{\perp}=0}
$$

which is the result (5.11) that we used in section 5 .

As an example of its use, the result (G.5) can be applied in a straightforward fashion in any case where the physics of momentum broadening can be understood as diffusion in $k_{\perp}$-space, as for example in the strong-coupling regime that we have discussed in section V.D. In any such context,

$$
\mathcal{W}_{\mathcal{R}}^{(2)}=-D x_{\perp}^{2}
$$

for some diffusion constant $D$, and applying (G.5) yields

$$
\hat{q}=\frac{4 D}{L}
$$

immediately. This is of course what we also find by applying the definition of $\hat{q}$ in eq. (G.1).

Applying the result (G.5) to the case of a weakly-coupled plasma, as we analyze in this paper, must come with a further subtlety since, as we have discussed in section V.C, in this case the jet quenching parameter $\hat{q}$ is not well defined! The result of the integration in eq. (G.1) is logarithmically divergent, meaning that it must be regulated in some way. Similarly, if we simply apply (G.5) to our weak-coupling result (2.18) for $\mathcal{W}_{\mathcal{R}}^{(2)}$ we find a divergent result. To regulate this divergence, we write the result (G.5) in Fourier space, and regulate the momentum integral by imposing a UV cutoff $\Lambda_{U V}$, as discussed in section V.C. Upon doing so with $\mathcal{W}_{\mathcal{R}}^{(2)}$ given by (2.18), we obtain

$$
\hat{q}=\frac{1}{L} \int^{\Lambda_{\mathrm{UV}}} \frac{d^{2} k_{\perp}}{(2 \pi)^{2}} k_{\perp}^{2} P_{\text {thin }}\left(k_{\perp}\right),
$$

which is the expression (5.13) that we used to evaluate the jet quenching parameter $\hat{q}$ in section V.C.

Open Access. This article is distributed under the terms of the Creative Commons Attribution License which permits any use, distribution and reproduction in any medium, provided the original author(s) and source are credited. 


\section{References}

[1] BRAHMS collaboration, I. Arsene et al., Transverse momentum spectra in $A u+A u$ and $d+A u$ collisions at $\sqrt{s}=200$ GeV and the pseudorapidity dependence of high $p_{T}$ suppression, Phys. Rev. Lett. 91 (2003) 072305 [nucl-ex/0307003] [INSPIRE].

[2] PHENIX collaboration, S. Adler et al., Absence of suppression in particle production at large transverse momentum in $\sqrt{s_{N N}}=200 \mathrm{GeV} d+$ Au collisions, Phys. Rev. Lett. 91 (2003) 072303 [nucl-ex/0306021] [INSPIRE].

[3] PHOBOS collaboration, B. Back et al., Centrality dependence of charged hadron transverse momentum spectra in $d+$ Au collisions at $\sqrt{s_{N N}}=200$ GeV, Phys. Rev. Lett. 91 (2003) 072302 [nucl-ex/0306025] [INSPIRE].

[4] STAR collaboration, J. Adams et al., Evidence from $d+$ Au measurements for final state suppression of high $p_{T}$ hadrons in Au+Au collisions at RHIC, Phys. Rev. Lett. 91 (2003) 072304 [nucl-ex/0306024] [INSPIRE].

[5] ATLAS collaboration, Observation of a centrality-dependent dijet asymmetry in lead-lead collisions at $\sqrt{s_{N N}}=2.77 \mathrm{TeV}$ with the ATLAS detector at the LHC, Phys. Rev. Lett. 105 (2010) 252303 [arXiv:1011.6182] [INSPIRE].

[6] ALICE collaboration, Suppression of charged particle production at large transverse momentum in central PbPb collisions at $\sqrt{s_{N N}}=2.76$ TeV, Phys. Lett. B 696 (2011) 30 [arXiv:1012.1004] [INSPIRE].

[7] CMS collaboration, Observation and studies of jet quenching in PbPb collisions at nucleon-nucleon center-of-mass energy $=2.76$ TeV, Phys. Rev. C 84 (2011) 024906 [arXiv:1102.1957] [INSPIRE].

[8] CMS collaboration, Study of high- $p_{T}$ charged particle suppression in PbPb compared to $p p$ collisions at $\sqrt{s_{N N}}=2.76$ TeV, Eur. Phys. J. C 72 (2012) 1945 [arXiv:1202.2554] [INSPIRE].

[9] CMS collaboration, Jet momentum dependence of jet quenching in $\mathrm{PbPb}$ collisions at $\sqrt{s_{N N}}=2.76 \mathrm{TeV}$, Phys. Lett. B 712 (2012) 176 [arXiv:1202.5022] [INSPIRE].

[10] CMS collaboration, Studies of jet quenching using isolated-photon+jet correlations in $\mathrm{PbPb}$ and pp collisions at $\sqrt{s_{N N}}=2.76$ TeV, Phys. Lett. B 718 (2013) 773 [arXiv:1205.0206] [INSPIRE].

[11] CMS collaboration, Measurement of jet fragmentation into charged particles in pp and $\mathrm{PbPb}$ collisions at $\sqrt{s_{N N}}=2.76 \mathrm{TeV}, \mathrm{JHEP} 10$ (2012) 087 [arXiv:1205.5872] [INSPIRE].

[12] ATLAS collaboration, Measurement of the jet radius and transverse momentum dependence of inclusive jet suppression in lead-lead collisions at $\sqrt{s_{N N}}=2.76$ TeV with the ATLAS detector, Phys. Lett. B 719 (2013) 220 [arXiv:1208.1967] [INSPIRE].

[13] R. Granier de Cassagnac, Overview of results on photon and electroweak boson production from the CMS collaboration, talk at Quark Matter 2012 conference, Washington DC U.S.A. August 2012.

[14] G. Roland, Experimental highlights from the CMS collaboration, talk at Quark Matter 2012 conference, Washington DC U.S.A. August 2012.

[15] M. Spousta, ATLAS jets, talk at Quark Matter 2012 conference, Washington DC U.S.A. August 2012. 
[16] P. Steinberg, Photons and electroweak probes in ATLAS, talk at Quark Matter 2012 conference, Washington DC U.S.A. August 2012.

[17] G. Veres, Overview of results on jets from the CMS collaboration, talk at Quark Matter 2012 conference, Washington DC U.S.A. August 2012.

[18] B. Wosiek, ATLAS highlights, talk at Quark Matter 2012 conference, Washington DC U.S.A. August 2012.

[19] M. McCumber, A technique for charm and beauty separation via DCA unfolding, talk at Quark Matter 2012 conference, Washington DC U.S.A. August 2012.

[20] T. Sakaguchi, PHENIX highlights, talk at Quark Matter 2012 conference, Washington DC U.S.A. August 2012.

[21] M. Gyulassy and X.-N. Wang, Multiple collisions and induced gluon bremsstrahlung in QCD, Nucl. Phys. B 420 (1994) 583 [nucl-th/9306003] [INSPIRE].

[22] R. Baier, Y.L. Dokshitzer, A.H. Mueller, S. Peigne and D. Schiff, Radiative energy loss of high-energy quarks and gluons in a finite volume quark-gluon plasma, Nucl. Phys. B 483 (1997) 291 [hep-ph/9607355] [INSPIRE].

[23] R. Baier, Y.L. Dokshitzer, A.H. Mueller, S. Peigne and D. Schiff, Radiative energy loss and $p_{T}$ broadening of high-energy partons in nuclei, Nucl. Phys. B 484 (1997) 265 [hep-ph/9608322] [INSPIRE].

[24] B. Zakharov, Radiative energy loss of high-energy quarks in finite size nuclear matter and quark-gluon plasma, JETP Lett. 65 (1997) 615 [hep-ph/9704255] [INSPIRE].

[25] U.A. Wiedemann, Gluon radiation off hard quarks in a nuclear environment: opacity expansion, Nucl. Phys. B 588 (2000) 303 [hep-ph/0005129] [INSPIRE].

[26] M. Gyulassy, P. Levai and I. Vitev, Non-Abelian energy loss at finite opacity, Phys. Rev. Lett. 85 (2000) 5535 [nucl-th/0005032] [INSPIRE].

[27] M. Gyulassy, P. Levai and I. Vitev, Reaction operator approach to non-Abelian energy loss, Nucl. Phys. B 594 (2001) 371 [nucl-th/0006010] [INSPIRE].

[28] X.-F. Guo and X.-N. Wang, Multiple scattering, parton energy loss and modified fragmentation functions in deeply inelastic eA scattering, Phys. Rev. Lett. 85 (2000) 3591 [hep-ph/0005044] [INSPIRE].

[29] X.-N. Wang and X.-F. Guo, Multiple parton scattering in nuclei: parton energy loss, Nucl. Phys. A 696 (2001) 788 [hep-ph/0102230] [INSPIRE].

[30] P.B. Arnold, G.D. Moore and L.G. Yaffe, Photon emission from ultrarelativistic plasmas, JHEP 11 (2001) 057 [hep-ph/0109064] [INSPIRE].

[31] P.B. Arnold, G.D. Moore and L.G. Yaffe, Photon emission from quark gluon plasma: complete leading order results, JHEP 12 (2001) 009 [hep-ph/0111107] [INSPIRE].

[32] P.B. Arnold, G.D. Moore and L.G. Yaffe, Photon and gluon emission in relativistic plasmas, JHEP 06 (2002) 030 [hep-ph/0204343] [INSPIRE].

[33] S. Jeon and G.D. Moore, Energy loss of leading partons in a thermal QCD medium, Phys. Rev. C 71 (2005) 034901 [hep-ph/0309332] [INSPIRE].

[34] S. Turbide, C. Gale, S. Jeon and G.D. Moore, Energy loss of leading hadrons and direct photon production in evolving quark-gluon plasma, Phys. Rev. C 72 (2005) 014906 [hep-ph/0502248] [INSPIRE]. 
[35] C.A. Salgado and U.A. Wiedemann, Calculating quenching weights, Phys. Rev. D 68 (2003) 014008 [hep-ph/0302184] [INSPIRE].

[36] A. Majumder and B. Müller, Higher twist jet broadening and classical propagation, Phys. Rev. C 77 (2008) 054903 [arXiv: 0705.1147] [INSPIRE].

[37] R. Baier, D. Schiff and B. Zakharov, Energy loss in perturbative QCD, Ann. Rev. Nucl. Part. Sci. 50 (2000) 37 [hep-ph/0002198] [INSPIRE].

[38] A. Kovner and U.A. Wiedemann, Gluon radiation and parton energy loss, hep-ph/0304151 [INSPIRE].

[39] M. Gyulassy, I. Vitev, X.-N. Wang and B.-W. Zhang, Jet quenching and radiative energy loss in dense nuclear matter, nucl-th/0302077 [INSPIRE].

[40] P. Jacobs and X.-N. Wang, Matter in extremis: ultrarelativistic nuclear collisions at RHIC, Prog. Part. Nucl. Phys. 54 (2005) 443 [hep-ph/0405125] [INSPIRE].

[41] J. Casalderrey-Solana and C.A. Salgado, Introductory lectures on jet quenching in heavy ion collisions, Acta Phys. Polon. B 38 (2007) 3731 [arXiv:0712.3443] [InSPIRE].

[42] A. Accardi, F. Arleo, W. Brooks, D. D'Enterria and V. Muccifora, Parton propagation and fragmentation in QCD matter, Riv. Nuovo Cim. 32 (2010) 439 [arXiv:0907.3534] [INSPIRE].

[43] U.A. Wiedemann, Jet quenching in heavy ion collisions, arXiv:0908.2306 [INSPIRE].

[44] A. Majumder and M. Van Leeuwen, The theory and phenomenology of perturbative QCD based jet quenching, Prog. Part. Nucl. Phys. A 66 (2011) 41 [arXiv:1002.2206] [INSPIRE].

[45] C.W. Bauer, S. Fleming and M.E. Luke, Summing Sudakov logarithms in $B \rightarrow X /$ s $\gamma$ in effective field theory, Phys. Rev. D 63 (2000) 014006 [hep-ph/0005275] [INSPIRE].

[46] C.W. Bauer, S. Fleming, D. Pirjol and I.W. Stewart, An effective field theory for collinear and soft gluons: heavy to light decays, Phys. Rev. D 63 (2001) 114020 [hep-ph/0011336] [INSPIRE].

[47] C.W. Bauer and I.W. Stewart, Invariant operators in collinear effective theory, Phys. Lett. B 516 (2001) 134 [hep-ph/0107001] [InSPIRE].

[48] C.W. Bauer, D. Pirjol and I.W. Stewart, Soft collinear factorization in effective field theory, Phys. Rev. D 65 (2002) 054022 [hep-ph/0109045] [INSPIRE].

[49] A. Idilbi and A. Majumder, Extending soft-collinear-effective-theory to describe hard jets in dense QCD media, Phys. Rev. D 80 (2009) 054022 [arXiv: 0808.1087] [INSPIRE].

[50] F. D'Eramo, H. Liu and K. Rajagopal, Transverse momentum broadening and the jet quenching parameter, redux, Phys. Rev. D 84 (2011) 065015 [arXiv:1006.1367] [InSPIRE].

[51] G. Ovanesyan and I. Vitev, An effective theory for jet propagation in dense QCD matter: jet broadening and medium-induced bremsstrahlung, JHEP 06 (2011) 080 [arXiv:1103.1074] [INSPIRE].

[52] G. Ovanesyan and I. Vitev, Medium-induced parton splitting kernels from soft collinear effective theory with Glauber gluons, Phys. Lett. B 706 (2012) 371 [arXiv:1109.5619] [INSPIRE].

[53] Z.-T. Liang, X.-N. Wang and J. Zhou, The transverse-momentum-dependent parton distribution function and jet transport in medium, Phys. Rev. D 77 (2008) 125010 [arXiv: 0801.0434] [INSPIRE]. 
[54] R. Baier, A. Kovner and U.A. Wiedemann, Saturation and parton level Cronin effect: enhancement versus suppression of gluon production in $p-A$ and $A$-A collisions, Phys. Rev. D 68 (2003) 054009 [hep-ph/0305265] [INSPIRE].

[55] J.L. Albacete, N. Armesto, A. Kovner, C.A. Salgado and U.A. Wiedemann, Energy dependence of the Cronin effect from nonlinear QCD evolution, Phys. Rev. Lett. 92 (2004) 082001 [hep-ph/0307179] [INSPIRE].

[56] D. Kharzeev, Y.V. Kovchegov and K. Tuchin, Cronin effect and high $p_{T}$ suppression in $p$ - $A$ collisions, Phys. Rev. D 68 (2003) 094013 [hep-ph/0307037] [INSPIRE].

[57] M. Benzke, N. Brambilla, M.A. Escobedo and A. Vairo, Gauge invariant definition of the jet quenching parameter, JHEP 02 (2013) 129 [arXiv:1208.4253] [INSPIRE].

[58] H. Liu, K. Rajagopal and U.A. Wiedemann, Calculating the jet quenching parameter from AdS/CFT, Phys. Rev. Lett. 97 (2006) 182301 [hep-ph/0605178] [INSPIRE].

[59] A.D. Linde, Infrared problem in thermodynamics of the Yang-Mills gas, Phys. Lett. B 96 (1980) 289 [INSPIRE].

[60] O. Kalashnikov and V. Klimov, Infrared behavior of Green functions in Yang-Mills theory at finite temperatures, Sov. J. Nucl. Phys. 33 (1981) 443 [Yad. Fiz. 33 (1980) 848] [INSPIRE].

[61] E. Braaten and R.D. Pisarski, Resummation and gauge invariance of the gluon damping rate in hot QCD, Phys. Rev. Lett. 64 (1990) 1338 [InSPIRE].

[62] J. Frenkel and J. Taylor, High temperature limit of thermal QCD, Nucl. Phys. B 334 (1990) 199 [INSPIRE].

[63] E. Braaten and R.D. Pisarski, Soft amplitudes in hot gauge theories: a general analysis, Nucl. Phys. B 337 (1990) 569 [INSPIRE].

[64] E. Braaten and R.D. Pisarski, Deducing hard thermal loops from Ward identities, Nucl. Phys. B 339 (1990) 310 [INSPIRE].

[65] E. Braaten and R.D. Pisarski, Simple effective Lagrangian for hard thermal loops, Phys. Rev. D 45 (1992) 1827 [INSPIRE].

[66] P.B. Arnold and C. Dogan, QCD splitting/joining functions at finite temperature in the deep LPM regime, Phys. Rev. D 78 (2008) 065008 [arXiv:0804.3359] [INSPIRE].

[67] P.B. Arnold and W. Xiao, High-energy jet quenching in weakly-coupled quark-gluon plasmas, Phys. Rev. D 78 (2008) 125008 [arXiv:0810.1026] [INSPIRE].

[68] S. Caron-Huot, $O(g)$ plasma effects in jet quenching, Phys. Rev. D 79 (2009) 065039 [arXiv:0811.1603] [INSPIRE].

[69] A. Majumder, B. Müller and S. Mrowczynski, Momentum broadening of a fast parton in a perturbative quark-gluon plasma, Phys. Rev. D 80 (2009) 125020 [arXiv:0903.3683] [INSPIRE].

[70] M. Le Bellac, Thermal field theory, Cambridge monographs on Mathematical Physics, Cambridge University Press, Cambridge U.K. (1996).

[71] K. Kajantie and J.I. Kapusta, Behavior of gluons at high temperature, Annals Phys. 160 (1985) 477 [INSPIRE].

[72] R. Kobes, G. Kunstatter and K. Mak, Linear response of the hot QCD plasma from the gluon propagator, Z. Phys. C 45 (1989) 129 [INSPIRE]. 
[73] H.A. Weldon, Structure of the gluon propagator at finite temperature, Annals Phys. 271 (1999) 141 [hep-ph/9701279] [INSPIRE].

[74] F.T. Brandt and J. Frenkel, Generalized forward scattering amplitudes in QCD at high temperature, Phys. Rev. D 56 (1997) 2453 [hep-th/9703165] [InSPIRE].

[75] O. Kalashnikov and V. Klimov, Polarization tensor in QCD for finite temperature and density, Sov. J. Nucl. Phys. 31 (1980) 699 [Yad. Fiz. 31 (1980) 1357] [INSPIRE].

[76] O. Kalashnikov, Magnetic mass in hot scalar electrodynamics, Kratk. Soobshch. Fiz. (1996) Nos. 7-8 52 [hep-ph/9602308] [INSPIRE].

[77] H.A. Weldon, Covariant calculations at finite temperature: the relativistic plasma, Phys. Rev. D 26 (1982) 1394 [inSPIRE].

[78] V. Klimov, Collective excitations in a hot quark gluon plasma, Sov. Phys. JETP 55 (1982) 199 [Zh. Eksp. Teor. Fiz. 82 (1982) 336] [InSPIRE].

[79] M. Laine, A non-perturbative contribution to jet quenching, Eur. Phys. J. C 72 (2012) 2233 [arXiv:1208.5707] [INSPIRE].

[80] P. Aurenche, F. Gelis and H. Zaraket, A simple sum rule for the thermal gluon spectral function and applications, JHEP 05 (2002) 043 [hep-ph/0204146] [INSPIRE].

[81] U.A. Wiedemann and M. Gyulassy, Transverse momentum dependence of the Landau-Pomeranchuk-Migdal effect, Nucl. Phys. B 560 (1999) 345 [hep-ph/9906257] [INSPIRE].

[82] M. Gyulassy, P. Levai and I. Vitev, Reaction operator approach to multiple elastic scatterings, Phys. Rev. D 66 (2002) 014005 [nucl-th/0201078] [InSPIRE].

[83] P.B. Arnold, Simple formula for high-energy gluon bremsstrahlung in a finite, expanding medium, Phys. Rev. D 79 (2009) 065025 [arXiv:0808.2767] [inSPIRE].

[84] A. Peshier, QCD running coupling and collisional jet quenching, J. Phys. G 35 (2008) 044028 [INSPIRE].

[85] J.M. Maldacena, The large- $N$ limit of superconformal field theories and supergravity, Adv. Theor. Math. Phys. 2 (1998) 231 [Int. J. Theor. Phys. 38 (1999) 1113] [hep-th/9711200] [INSPIRE].

[86] S. Gubser, I.R. Klebanov and A.M. Polyakov, Gauge theory correlators from noncritical string theory, Phys. Lett. B 428 (1998) 105 [hep-th/9802109] [INSPIRE].

[87] E. Witten, Anti-de Sitter space, thermal phase transition and confinement in gauge theories, Adv. Theor. Math. Phys. 2 (1998) 505 [hep-th/9803131] [INSPIRE].

[88] J.M. Maldacena, Wilson loops in large-N field theories, Phys. Rev. Lett. 80 (1998) 4859 [hep-th/9803002] [INSPIRE].

[89] S.-J. Rey and J.-T. Yee, Macroscopic strings as heavy quarks in large- $N$ gauge theory and anti-de Sitter supergravity, Eur. Phys. J. C 22 (2001) 379 [hep-th/9803001] [InSPIRE].

[90] S.-J. Rey, S. Theisen and J.-T. Yee, Wilson-Polyakov loop at finite temperature in large- $N$ gauge theory and anti-de Sitter supergravity, Nucl. Phys. B 527 (1998) 171 [hep-th/9803135] [INSPIRE].

[91] A. Brandhuber, N. Itzhaki, J. Sonnenschein and S. Yankielowicz, Wilson loops in the large- $N$ limit at finite temperature, Phys. Lett. B 434 (1998) 36 [hep-th/9803137] [INSPIRE]. 
[92] J. Sonnenschein, What does the string/gauge correspondence teach us about Wilson loops?, hep-th/0003032 [INSPIRE].

[93] J. Casalderrey-Solana, H. Liu, D. Mateos, K. Rajagopal and U.A. Wiedemann, Gauge/string duality, hot QCD and heavy ion collisions, arXiv:1101.0618 [INSPIRE].

[94] M.H. Thoma, New developments and applications of thermal field theory, hep-ph/0010164 [INSPIRE].

[95] Z. Ligeti, I.W. Stewart and F.J. Tackmann, Treating the $b$ quark distribution function with reliable uncertainties, Phys. Rev. D 78 (2008) 114014 [arXiv:0807.1926] [INSPIRE].

[96] I.W. Stewart, F.J. Tackmann and W.J. Waalewijn, The quark beam function at NNLL, JHEP 09 (2010) 005 [arXiv: 1002.2213] [InSPIRE].

[97] G. Molière, Theorie der Streuung schneller geladener Teilchen II. Mehrfach- und Vielfachstreuung (in German), Z. Naturforsch. 3a (1948) 78.

[98] H. Bethe, Moliere's theory of multiple scattering, Phys. Rev. 89 (1953) 1256 [InSPIRE].

[99] B.G. Zakharov, Applicability of the eikonal approximation for calculation of the probability of passage of ultrarelativistic positronium through matter, Sov. J. Nucl. Phys. 46 (1987) 92.

[100] N.N. Nikolaev and B. Zakharov, Color transparency and scaling properties of nuclear shadowing in deep inelastic scattering, Z. Phys. C 49 (1991) 607 [InSPIRE]. 